\title{
Mechanisms of Turbulent Mixing in the Continental Shelf Bottom Boundary Layer
}

\author{
By \\ William J. Shaw \\ B.S.E., Princeton University, 1993 \\ Submitted in partial fulfillment of the requirements for the dual degrees of \\ Doctor of Philosophy \\ at the \\ MASSACHUSETTS INSTITUTE OF TECHNOLOGY \\ and the \\ WOODS HOLE OCEANOGRAPHIC INSTITUTION \\ September 1999 \\ (C)1999 William J. Shaw \\ All rights reserved \\ The author hereby grants to MIT and WHOI permission to reproduce paper and \\ electronic copies of this thesis in whole or in part and to distribute publicly.
}

Signature of Author

Joint Program in Oceanography Massachusetts Institute of Technology and Woods Hole Oceanographic Institution September 1999

Certified by

John H. Trowbridge, Thesis Supervisor

Accepted by

Deborah K. Smith

Chair, Joint Committee for Marine Geology and Geophysics Woods Hole Oceanographic Institution 



\title{
Mechanisms of Turbulent Mixing in the Continental Shelf Bottom Boundary Layer
}

\author{
By \\ William J. Shaw \\ Submitted to the Woods Hole Oceanographic Institution / Massachusetts Institute of \\ Technology Joint Program in Marine Geology and Geophysics in September, 1999 in \\ partial fulfillment of the requirements for the degree of Doctor of Philosophy.
}

\begin{abstract}
The bottom boundary layer is an important dynamical region of shallow water flows. In this thesis, the problem of turbulent mixing in the coastal bottom boundary layer is investigated with a unique set of field measurements of velocity and sound speed that span a significant fraction of the boundary layer obtained over a six-week long period in the late summer of 1996 on the New England shelf. The energetics of the turbulent fluctuations are investigated by testing simplified budgets for turbulent kinetic energy and scalar variance. The turbulent kinetic energy budget is locally balanced while the scalar variance budget is not, probably due to turbulent diffusion. The direct effects of stratification are consistently significant only in the outer part of the boundary layer, where the flux Richardson number is approximately equal to a critical value of 0.2 . Turbulence closure is investigated in terms of non-dimensional profiles of velocity and sound speed. Close to the bottom, the results are consistent with Monin-Obukhov similarity theory, while in the outer part of the boundary layer other scales including the height of the boundary layer are important for setting the turbulent length scale.
\end{abstract}

Thesis Supervisor: John H. Trowbridge, Associate Scientist, WHOI 


\section{Acknowledgements}

The completion of this thesis would not have been possible without the help of a long list of people. First and foremost, I thank John Trowbridge, my advisor, for giving me a chance to work with him in the beginning and for providing me with an excellent apprenticeship. I thank Sandy Williams for his mentorship, support, and encouragement. I thank the members of my thesis committee, Dave Aubrey, Jim Edson, Ole Madsen, Ray Schmitt and Steve Lentz, for their participation and all their helpful input. Janet Fredericks shared her BASS data processing knowledge with me and solved an uncountable number of my computer problems. The work I did would not have been possible without the expertise of everyone who was involved in the design, construction, deployment and recovery of the 'SuperBASS' tripod. I thank all of the Coastal Mixing and Optics investigators who gave me access to their data. In the beginning of my graduate student career, Jian Lin provided support for me as well as excellent guidance.

I would like to thank all of my friends in the Woods Hole and Cambridge communities for making my graduate student career a most enjoyable experience. I would especially like to thank all of the folks who have been affiliated in one way or another over the years with a particular address on Millfield St., Woods Hole. I am grateful for the companionship of Lihini and I am indebted to my mother and father for their unwavering support of my education.

My doctoral work was supported by the Office of Naval Research under grants N000149510373 and N000149610953. 


\section{Contents}

1 Introduction

2 Measurement of Turbulent Sound Speed Fluctuations with an Acoustic

$\begin{array}{ll}\text { Travel-Time Current Meter } & 21\end{array}$

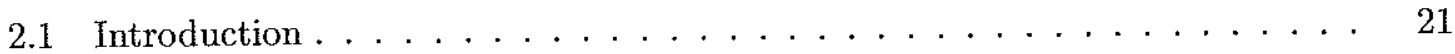

2.2 BASS Description . . . . . . . . . . . . . . . 24

2.3 Hudmix Analysis . . . . . . . . . . . . . . . . . 28

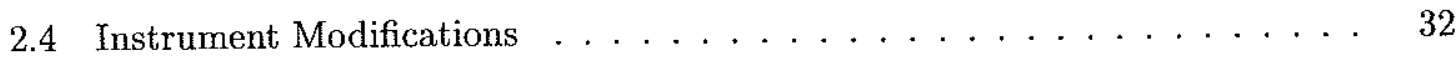

2.5 Conclusions . . . . . . . . . . . . . . . 37

3 The Direct Estimation of Near-bottom Turbulent Fluxes in the Presence of Energetic Wave Motions

3.1 Introduction . . . . . . . . . . . . . . . 44

3.2 Theoretical Framework . . . . . . . . . . . . . . . 47

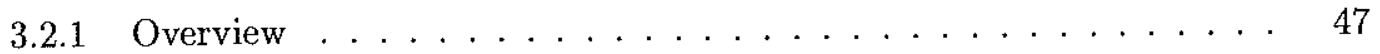

3.2 .2 Differencing Strategies . . . . . . . . . . . . . . 51

3.2.3 Further Reduction in Wave Bias with Adaptive Filtering . . . . . . . 55 
3.3 Measurements and Analysis . . . . . . . . . . . . . . . . 59

3.4 Results . . . . . . . . . . . . . . . . . . . . 62

3.4 .1 Conditions at the CMO Site . . . . . . . . . . . . . 62

3.4 .2 Shear Stress . . . . . . . . . . . . . . . . . 64

3.4 .3 Heat Flux . . . . . . . . . . . . . . 75

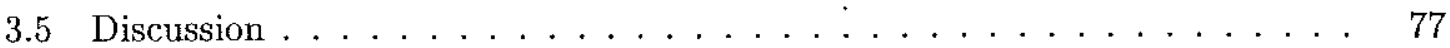

3.6 Summary and Conclusions . . . . . . . . . . . . . . 83

4 The Budgets of Turbulent Kinetic Energy and Scalar Variance in the $\begin{array}{lr}\text { Continental Shelf Bottom Boundary Layer } & 89\end{array}$

4.1 Introduction . . . . . . . . . . . . . . . 90

4.2 Methods . . . . . . . . . . . . . . . . . . . . . 94

4.2 .1 Measurements . . . . . . . . . . . . . . . . . 94

$4.2 .2 \quad$ Flow Disturbances and Instrument Problems $\ldots \ldots \ldots$. . . . . . 99

4.2 .3 Dissipation Rate Estimates . . . . . . . . . . . . . . . . . 101

4.2 .4 Flux Estimates . . . . . . . . . . . . . . 105

4.3 Results. . . . . . . . . . . . . . . . . . . . 110

4.3.1 Conditions at the CMO Site . . . . . . . . . . . . 110

4.3 .2 TKE budget $\ldots \ldots \ldots \ldots \ldots \ldots \ldots$

4.3 .3 TSV budget . . . . . . . . . . . . . . . 115

4.4 Discussion . . . . . . . . . . . . . . . . 117

4.5 Summary and Conclusions . . . . . . . . . . . . . . . . . 123

5 Flux-Profile Relationships in the Continental Shelf Bottom Boundary 
5.1 Introduction . . . . . . . . . . . . . . . . . 130

5.2 Similarity Theory . . . . . . . . . . . . . . . 133

5.2 .1 Monin-Obukhov Similarity . . . . . . . . . . 136

5.2 .2 Boundary Layer Height Scaling . . . . . . . . . . . . . 137

5.3 Methods . . . . . . . . . . . . . . . . 138

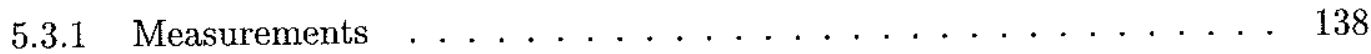

5.3 .2 Boundary Layer Estimates . . . . . . . . . . . . . . 139

5.4 Results. . . . . . . . . . . . . . . . . . . . . . 141

5.4 .1 Site Conditions . . . . . . . . . . . . . . . . . 141

5.4 .2 Flux Estimates . . . . . . . . . . . . . . . 145

5.4 .3 Dimensionless Gradients . . . . . . . . . . . . . . . 150

5.5 Discussion . . . . . . . . . . . . . . . 158

5.6 Summary and Conclusions . . . . . . . . . . . . . . . 164

6 Summary $\quad 169$ 


\section{Chapter 1}

\section{Introduction}

Continental shelves are shallow, gently sloping regions extending from the coast to a depth of about $200 \mathrm{~m}$ at the shelf break. Shelf widths range from 20 to $150 \mathrm{~km}$ with an average value of approximately $65 \mathrm{~km}$. Shelf flows are affected by a number of mechanisms including winds, tides, and density differences and the relative importance of these factors varies geographically and seasonally. Rivers empty onto continental shelves, affecting the density structure and providing a major source of sediment. The bathymetry of continental shelves is responsible for unique dynamics not present in the deep ocean. For example, the boundary condition imposed by the coast allows upwelling and downwelling, and the relative importance of friction is greater in the shallow water than in the deep ocean.

An important dynamical region of shelf flows is the turbulent flow of the bottom boundary layer (BBL) that results from bottom drag. The flux of momentum to the sea floor has a significant impact on shelf dynamics; it is an important term, for example, in vertically integrated momentum equations. Turbulent mixing also enhances rates of energy dissipation and, because tidal velocities are large on the shelf, a significant portion of the oceans' tidal energy is dissipated in the shelf BBL. In the context of upwelling and downwelling dynamics, the BBL is a potentially important conduit for cross shelf exchange. The shelf BBL is also 
the interface between the sea floor and the overlying water column. The erosion, deposition and transport of sediment occurs within the BBL. In general, the bottom boundary layer is an important source of mixing with implications for many aspects of coastal oceanography.

To capture the effects of turbulent mixing, models require parameterizations of the turbulent fluxes of momentum and scalars such as heat, salt or concentration of suspended sediment. Oceanic BBL models have used a wide range of closure schemes varying in complexity from simple bulk Richardson number mixing criteria [Thompson, 1973, Trowbridge and Lentz, 1991] and eddy viscosity models [Soulsby, 1983, Garrett et al., 1993] to complicated second-order closures [Weatherly and Martin, 1978]. A common feature of all the models is a reliance on semi-empirical theories that have not been rigorously tested in the shelf BBL.

More fundamentally, it may be stated that a number of important problems in the dynamics of turbulence in the shelf BBL remain unaddressed, due to a lack of direct measurement. These questions include the potential importance of coherent motions in transporting momentum and scalars, the non-linear interaction between various forcing mechanisms and determining the turbulent length scale. The turbulent length scale is of central importance because of its role in gradient transport models of turbulent diffusion, such as the mixing length theory of Prandtl [Tennekes and Lumley, 1972]. In order for the mixing length concept to be valid, the turbulence should, at a minimum, be locally balanced. If turbulence is essentially local, it is important to know what variables control the mixing length and if the mixing lengths for momentum and scalars are essentially equal. If the turbulence is not locally balanced, it is important to know what mechanisms are responsible.

Throughout the present century, a central goal of turbulent boundary layer research 
has been to provide the semi-empirical hypotheses required to describe engineering and geophysical boundary layers. The most universal result from the previous work in wallbounded shear flows indicates that close, but not too close, to the bottom, the turbulent length scale is proportional to the distance from the boundary and the Karman-Prandtl law of the wall is satisfied [Monin and Yaglom, 1971]. Further from the boundary, scales associated with the height of the boundary layer [Coles, 1956], stratification [Obukhov, 1949] and pressure gradients [Yaglom, 1979] are expected to be important. length scale. Detailed descriptions of the outer part of the boundary layer are expected to be specific to the particular case being studied.

Although the shelf BBL may be expected to share some of the classical characteristics of other wall-bounded shear flows, it does have unique features. Density stratification is potentially important in the oceanic BBL and, in contrast to the atmospheric surface boundary layer, the dominant source of buoyant forcing is not surface heating or cooling due to the fact that surface fluxes of heat and salinity in the oceanic case are negligible [Wimbush and Munk, 1970]. It is expected, therefore, that processes such as entrainment at the top of the boundary layer and horizontal advection of stratification are important sources of buoyancy forcing. The sea floor is erodible and can also serve as a source of buoyancy forcing.

The earliest direct measurements of bottom stress in the oceanic BBL in 1950's [Bowden and Fairbairn, 1952, 1956] were used to determine values of the bottom drag coefficient necessary to estimate the rate of energy dissipation on continental shelves and for use in models with drag law closures. The early studies also considered the scale issue by estimating integral length scales. Later studies documented the similarity of the oceanic BBL 
to other boundary layers in terms of relationships between the magnitudes of the Reynolds stress tensor [Gordon and Dohne, 1973, Gross and Nowell, 1983], the occurrence of the bursting phenomenon [Gordon, 1974, Heathershaw, 1979] and spectral similarity [Soulsby, 1977, Heathershaw, 1979]. Only a few studies have obtained the necessary measurements to test simplified variance budgets [Gross and Nowell, 1985, Trowbridge et al., in press] or the Prandtl-Karman law of the wall [Smith and McLean, 1977, Soulsby and Dyer, 1979, Gross and Nowell, 1983, Trowbridge et al., in press].

All of the measurements to date have been limited to strong tidal flows. Most of the studies have also been limited to positions within a few meters of the bottom. There has been only a single attempt to directly measured the effect of stratification on coastal BBL turbulence [Kawanisi and Yokosi, 1994]. On the open continental shelf, where surface wave motions are capable of penetrating to the bottom, it has been impossible to obtain direct measurements of turbulent fluxes due to contamination by wave-induced fluctuations. As a whole, the available oceanic BBL measurements indicate that there is a vaguely defined near-bottom region that is similar in many respects to other boundary layer flows. The outer part of the coastal BBL and the dynamics of scalar fluctuations are largely unexplored.

The goals of the present thesis are to use novel measurement and analysis techniques to obtain direct (or nearly direct) measurements of BBL turbulence on the continental shelf and to use these measurements to study the mechanisms of turbulent mixing. In particular, the technical goal is to measure all of the terms in the local budgets for turbulent kinetic energy and the variance of sound speed (a surrogate for temperature). The thesis has two broad scientific goals. The first is to use the variance budgets to test if the turbulence is in local balance and to asses the importance of local stratification in terms of flux Richardson 
number. The second goal is to determine the dependence of the PrandtI mixing length on the distance from the bottom, the Monin-Obukhov length and the height of the boundary layer and to determine if the mixing length for momentum and scalars are equal.

The remainder of the thesis is organized into four research papers and a short concluding section. The first paper details modifications made to the Benthic Acoustic Stress Sensor to make it capable of resolving turbulent sound speed fluctuations. The second paper introduces a technique for obtaining near-bottom turbulent flux estimates in the presence of energetic surface waves. The third paper describes the variance budgets of turbulent kinetic energy and sound speed. The fourth paper describes the relationships between mean gradients and turbulent fluxes in terms of the turbulent length scale and the turbulent Prandtl number. 


\section{Bibliography}

K. F. Bowden and L. A. Fairbairn. A determination of the frictional forces in a tidal current. Proc. Roy. Soc. A, 214:371-391, 1952.

K. F. Bowden and L. A. Fairbairn. Measurements of turbulent fluctuations and reynold's stresses in a tidal current. Proc. Roy. Soc. A, 237:422-438, 1956.

D. Coles. The law of the wake in the turbulent boundary layer. J. Fluid Mech., 2:191-226, 1956.

C. Garrett, P. MacCready, and P. Rhines. Boundary mixing and arrested ekman layers. Annu. Rev. Fluid Mech., 25:291-323, 1993.

C. M. Gordon. Intermittent momentum transport in a tidal boundary layer. Nature, 248: $392-394,1974$.

C. M. Gordon and C. F. Dohne. Some observation of turbulence in a tidal estuary. $J$. Geophys. Res., 78:1971-1978, 1973.

T. F. Gross and A. R. M. Nowell. Mean flow and turbulence scaling in a tidal boundary layer. Cont. Shelf Res., 2:109-126, 1983. 
T. F. Gross and A. R. M. Nowell. Spectral scaling in a tidal boundary layer. J. Phys. Oceanogr., 15:496-508, 1985.

A. D. Heathershaw. The turbulent structure of the bottom boundary layer in a tidal current. Geophys. J. R. Astr. Soc., 58:395-430, 1979.

K. Kawanisi and S. Yokosi. Mean and turbulence characteristics in a tidal river. Est. Coast. Shelf Sci., 38:447-469, 1994.

A. S. Monin and A. M. Yaglom. Statistical Fluid Mecahnics. MIT Press, Cambridge, Mass., 1971.

A. M. Obukhov. Turbulence in an atmosphere with a non-uniform temperature. Trudy Inst. Teoret. Geofiz. Nauk SSSR, 1:95-115, 1949.

J. D. Smith and S. R. McLean. Spatially averaged flow over a wavy surface. J. Geophys. Res., 82:1735-1746, 1977.

R. L. Soulsby. Similarity scaling of turbulence spectra in marine and atmospheric boundary layers. J. Phys. Oceanog., 7:934-937, 1977.

R. L. Soulsby. The bottom boundary layer of shelf seas. In B. Johns, editor, Physical Oceanography of Coastal and Shelf Seas, pages 189-266. Elsevier, 1983.

R. L. Soulsby and K. R. Dyer. The form of the near-bed velocity profile in a tidally accelerating flow. J. Geophys. Res., 86:8067-8074, 1979.

H. Tennekes and J. L. Lumley. A First Course in Turbulence. MIT Press, Cambridge, Mass., 1972. 
R. O. R. Y. Thompson. Stratified ekman boundary layer models. Geophys. Fluid Dyn., 5: 201-210, 1973.

J. H. Trowbridge, W. R. Geyer, M. M. Bowen, and A. J. Williams. Near-bottom turbulence mesurements in a partially mixed estuary: Turbulent energy balance, velocity structure, and along-channel momentum balance. J. Phys. Oceanogr., in press.

J. H. Trowbridge and S. J. Lentz. Asymetric behavior of an oceanic boundary layer above a sloping bottom. J. Phys. Oceang., 21:1171-1185, 1991.

G. L. Weatherly and P. J. Martin. On the strucutre and dynamics of the oceanic bottom boundary layer. J. Phys. Oceanogr., 5:557-570, 1978.

M Wimbush and W. H. Munk. The benthic boundary layer. In The Sea, volume 4. WileyInterscience, 1970.

A. M. Yaglom. Similarity laws for constant-pressure and pressure-gradient turbulent wall flows. Ann. Rev. Fluid Mech., 11:505-540, 1979. 


\title{
Chapter 2
}

\section{Measurement of Turbulent Sound Speed Fluctuations with an Acoustic Travel-Time Current Meter}

\author{
William J. Shaw, Albert J. Williams III and John H. Trowbridge \\ (This chapter represents a paper that appeared in The Proceedings of the Oceans 96 \\ MTS/IEEE Meeting, 1996.)
}

\begin{abstract}
An acoustic current meter measuring absolute and differential travel-time of sound through water was deployed in the Hudson Estuary. One goal of the experiment was to make direct estimates of buoyancy fluxes. Results indicate that the absolute travel-time measurements were reliable in a mean sense but that the fluctuations were dominated by an electronic oscillation and a high noise floor. The absolute travel- time circuitry has been modified to remove the oscillation and reduce the noise floor. The oscillation has been almost removed and the noise floor has been reduced by more than an order of magnitude. We expect that buoyancy fluxes will be resolved in future developments if a record of sufficient length is obtained.
\end{abstract}

\subsection{Introduction}

Oceanic bottom boundary layers are regions of turbulent mixing of mass, momentum and heat, and are responsible for a large fraction of energy dissipation in the ocean. They also 
comprise interfaces across which particles, chemicals and organisms are exchanged between the sea floor and the overlying water column. Yet, the relative importance of various processes contributing to the dynamics of the oceanic bottom boundary layer is still generally unknown. Close to the boundary, there exists a layer in which classical scaling relations determined in laboratory experiments and atmospheric studies [Monin and Yaglom, 1971] may reasonably be assumed to apply. Far from the boundary, in the outer portion of the boundary layer, classical scalings are inappropriate and buoyancy effects are expected to assume an important role. Many previous studies in the oceanic bottom boundary layer lacked the instrumentation necessary to measure turbulent quantities directly. Without direct turbulence measurements, researchers relied on scaling relations that were largely untested at the bottom of the ocean. A limited number of studies have begun to explore the applicability of these scaling relations in the oceanic bottom boundary layer. The outer portion of the oceanic bottom boundary layer remains largely unexplored. Direct measurements are especially important in the coastal ocean because of the number and variety of external forcings affecting its dynamics.

The Benthic Acoustic Stress Sensor (BASS) is capable of making direct turbulence measurements [Williams et al., 1987]. BASS provides high-quality measurements of mean and fluctuating water velocity based on the difference in acoustic travel-time between pairs of transducers. Recently, the BASS electronics have been modified to measure absolute, in addition to differential, travel time [Trivett, 1991]. In principle, absolute travel- time can be used to make acoustic estimates of temperature and buoyancy because the temperature dependence of sound speed in sea water [MacKenzie, 1981] is well established.

An instrument such as BASS can be used to obtain records of velocity fluctuations. In 
principle, momentum fluxes can be estimated directly by computing the cross-correlations between the fluctuating velocity components. Mechanical dissipation can be directly estimated using Kolmogorov's theory of the inertial subrange [e.g., Monin and Yaglom, 1971], which relates the energy density in a certain range of scales to the dissipation rate. Measurement of buoyancy fluxes requires high-quality measurement of temperature fluctuations. Furthermore, the temperature and velocity measurements must be done in the same volume. For this reason, the use of BASS to measure velocity and temperature fluctuations could have great utility. In principle, BASS can be used to directly estimate temperature flux and indirectly measure temperature dissipation. Thus, utilizing BASS's capabilities as an absolute travel-time meter may allow estimation of buoyancy flux, heat flux, and temperature variance dissipation in addition to the momentum flux and mechanical energy dissipation that is usually measure with BASS.

This paper describes efforts made to employ BASS as a high-precision, high frequency absolute travel-time meter in the bottom boundary layer of the coastal ocean. In the following, we first present background material on the functioning of BASS (Section 2.2). We then discuss problems encountered in the analysis of absolute travel-time data collected in the Hudson Estuary (Section 2.3). Finally, we describe modifications made to BASS to improve its performance as an absolute travel-time meter (Section 2.4) and present conclusions (Section 2.5). 


\subsection{BASS Description}

BASS measures differential and absolute travel-time by timing acoustic pulses traveling in opposite directions between two transducers. A single BASS sensor consists of four pairs of transducers held rigidly in place by a supporting cage (Figure 2.1). The path length between opposite transducers is $15 \mathrm{~cm}$. The four transducer pairs resolve the threedimensional velocity vector with one degree of redundancy. Individual BASS pods are normally mounted in vertical arrays within bottom tripods. Although the utility of BASS is not confined to the bottom boundary layer, the motion of the instrument relative to an absolute reference frame must be known to make accurate measurements.

A pulse of acoustic energy travels through a fluid medium at the speed of sound with respect to the fluid. Sound speed and flow velocity can be determined by accurately measuring the travel times of pulses between two fixed transducers. To first order, the water velocity and sound speed are [Williams et al., 1987]

$$
v_{p}=\frac{c^{2}}{2 d}\left(t_{1}-t_{2}\right)
$$

and

$$
c=\frac{2 d}{\left(t_{1}+t_{2}\right)}
$$

Here, $d$ is the BASS path length $(15 \mathrm{~cm}), v_{p}$ is the component of velocity along the path, $c$ is the local speed of sound, and $t_{1}$ and $t_{2}$ are the travel-times in opposite directions along the acoustic path. The relative error of these approximations is of order $v_{p}^{2} / c^{2}$, which is less than one part per million for typical ocean flows. 


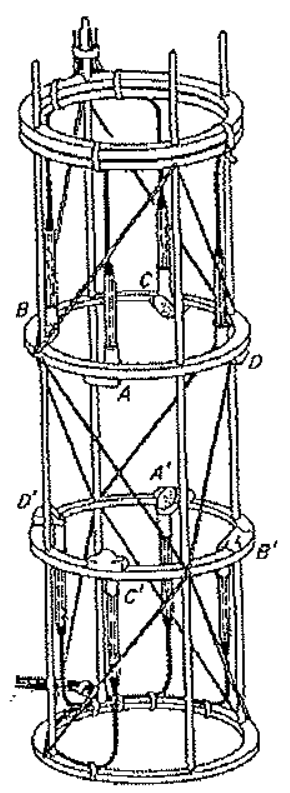

PERSPECTIVE VIEW

Figure 2.1: A schematic drawing of a single BASS sensor. Transducers on the upper ring $(\mathrm{A}, \mathrm{B}, \mathrm{C}$ and $\mathrm{D})$ form acoustic axes with transducers mounted diagonally opposite to them on the bottom ring (A', B', C' and $\left.D^{\prime}\right)$. 
When requested to make a measurement, BASS initiates a timing cycle controlling the transmission and measurement of the acoustic pulses traveling between a transducer pair (Figure 2.2, top). In the following discussion, the times at which specific events occur are given italicized names. For example, start-timing is the $t=0$ point of the cycle. At start-transmit, the beginning of a square-wave enveloped sinusoidal pulse is simultaneously transmitted by a pair of transducers. The pulses propagate along the acoustic path and are received by the opposite transducers with some attenuation. The 14th negative-going zero crossing is defined as the arrival time of a pulse by the trigger circuits following the transducers (Figure 2.2, bottom). As illustrated in Figure 2.2, the absolute travel-time between the lower and upper transducer $t_{1}$ and the absolute travel-time between the upper and lower transducer $t_{2}$ are

$$
t_{1}=(14 \text { th-received-upper })-(14 \text { th-transmitted })
$$

and

$$
t_{2}=(14 \text { th-received-lower })-(14 t h-\text { transmitted })
$$

respectively. For the geometry of BASS and typical sea water sound speed, the travel-times are approximately $100 \mu \mathrm{s}$.

BASS measures travel-times with an integrating circuit. At the desired starting time, a constant current source is switched from ground to a capacitor. At the desired stopping time, the current source is switched back to ground. The resulting capacitor voltage is proportional to the difference between the stop and start times.

To make a measurement of $v_{p}$, two integrating circuits are used to measure the differen- 

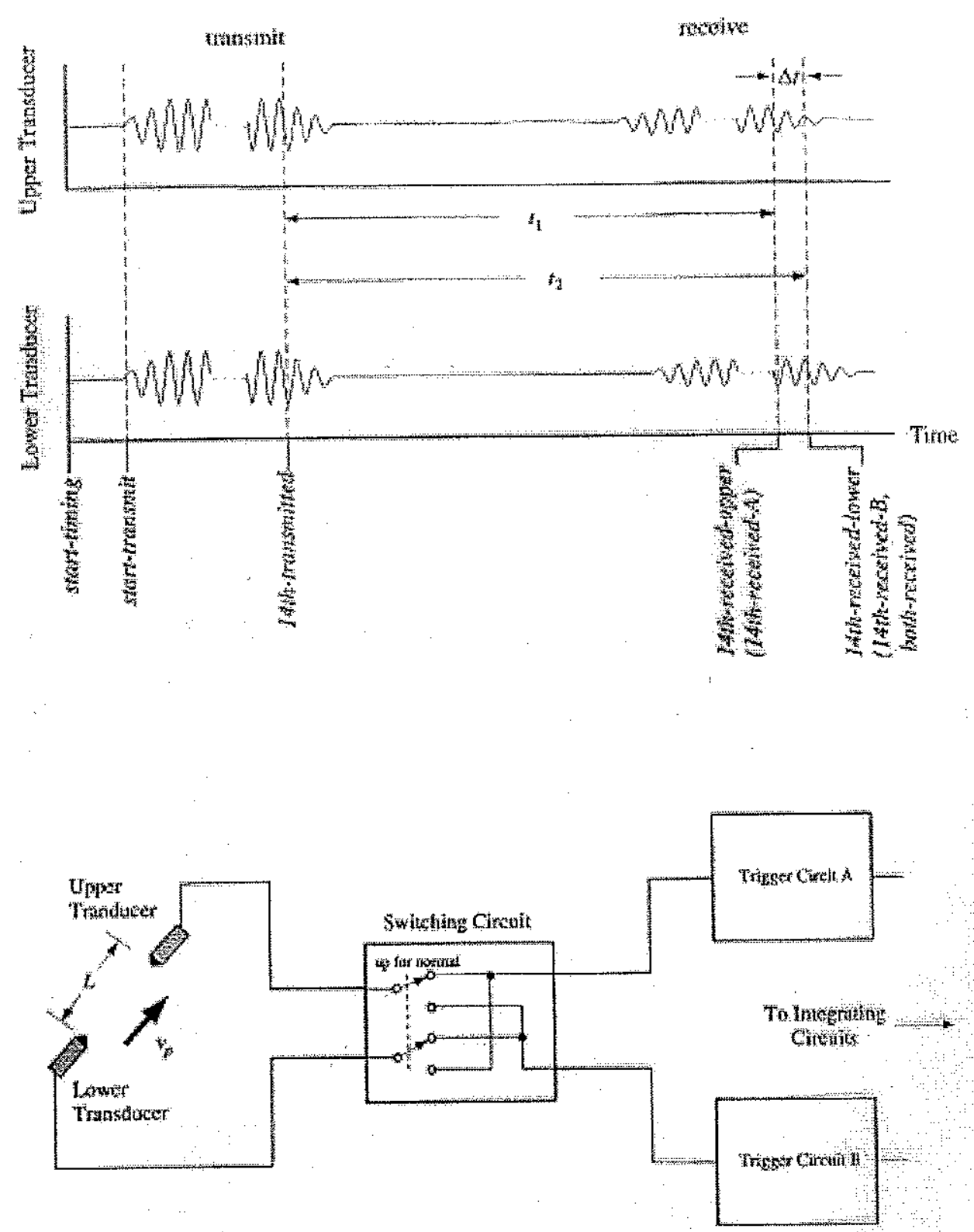

Figure 2.2: Top, diagram illustrating the transmitted and received pulses on a pair of BASS transducers. Each transducer first acts as a transmitter and then as a receiver for the other's transmitted pulse. Bottom, cartoon illustrating the receiving side of BASS electronics. For the situation drawn at bottom (flow velocity towards upper transducer and switch in normal polarity) the acoustic pulse traveling to the upper transducer arrives first and is detected by Trigger Circuit A. Both-received occurs a short time later when the pulse traveling to the lower circuit is detected by Trigger Circuit B. 
tial travel-times $\Delta t=t_{1}-t_{2}$. To reduce electronic errors, BASS repeats the measurement with the switching circuit between transducers and trigger circuits reversed (Figure 2.2, bottom). The sign of errors accumulated beyond the switch is independent of the polarity of the switch, but the sign of $\Delta t$ depends on the polarity of the switch. Thus, so long as the error does not change more rapidly that the interval between forward and reverse measurements, subtraction of normal and reverse measurements cancel noise that is generated beyond the switching point. The accuracy of the differential travel-time circuit is $40 \mathrm{ps}$ [Williams et al., 1987] corresponding to a flow velocity error of $0.3 \mathrm{~mm} / \mathrm{s}$.

To make a measurement of $c, t_{1}$ and $t_{2}$ must be measured as opposed to a direct measurement of $\Delta t$. The original absolute travel time circuit design [Trivett, 1991]started a single integrating circuit $85 \mu$ s into the timing cycle at unclamp. The integrating circuit was stopped when the slower pulse arrived, both-received (Figure 2.2 and Figure 2.3, top). Because $c$ is unaffected by switching polarity, the noise-reduction technique of subtracting normal and reverse measurements cannot be exploited in an absolute travel-time circuit. For this reason, the accurate measurement of absolute travel-time is expected to be more difficult than the accurate measurement of differential travel-time.

\section{$2.3 \quad$ Hudmix Analysis}

In late summer 1995, a tripod containing a vertical array of five BASS sensors, three thermistors, five conductivity cells, and a pressure sensor was deployed in the lower Hudson Estuary. The tripod deployment was part of the larger field program (Hudmix) aimed at identifying and quantifying vertical mixing rates in the Hudson estuary. Sampling was car- 


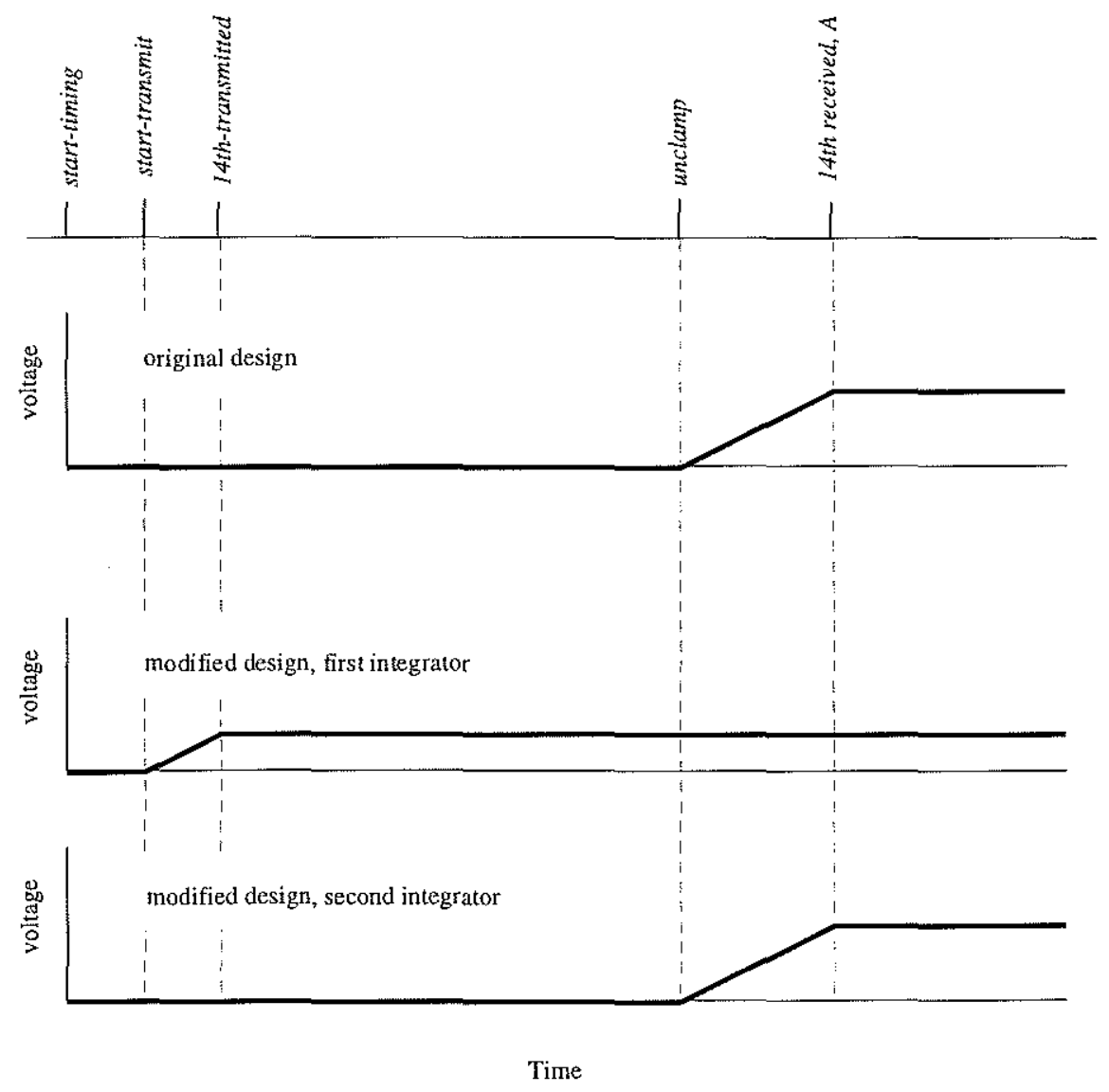

Figure 2.3: Timing Diagrams of absolute travel-time circuits. The voltage on the integrating capacitors is plotted as a function of time. Top, original design. Bottom, modified design. 
ried out at $6.25 \mathrm{~Hz}$ during 3.28 minute bursts every ten minutes. Absolute travel-time data were recorded in the normal direction only for one transducer pair of each sensor. Individual bursts were Reynolds averaged.

The output of BASS's absolute travel-time circuit is digitized counts corresponding to the voltage on the integrating capacitor. However, the absolute travel-time circuit was not calibrated. Therefore, the first step of the analysis was to obtain a calibration relating counts to sound speed. Since only $t_{1}$ was measured, sound speed is determined by

$$
v_{p}+c=\frac{d}{t_{1}}
$$

If $(2.5)$ is linearized for small sound speed fluctuations, the result is

$$
c=2 c_{0}-\frac{c_{0}^{2}}{d} t_{1}-v_{p}
$$

where $c_{0}$ is a constant reference sound speed. The voltages measured by BASS were calibrated by linearly regressing means of counts and predicted sound speeds (Figure 2.4). Predicted sound speeds were calculated with measured means of temperature, salinity and pressure from an empirical state equation for sea water [MacKenzie, 1981],

$$
\begin{aligned}
c= & 1448.96+4.591 T-5.304 \times 10^{-2} T^{2}+ \\
& 2.374 \times 10^{-4} T^{3}+1.340(s-35)+ \\
& 1.630 \times 10^{-2} D+1.675 \times 10^{-7} D^{2}- \\
& 1.025 \times 10^{-2} T(s-35)-7.139 \times 10^{-13} T D^{3} \pm 0.0070 \mathrm{~m} / \mathrm{s},
\end{aligned}
$$




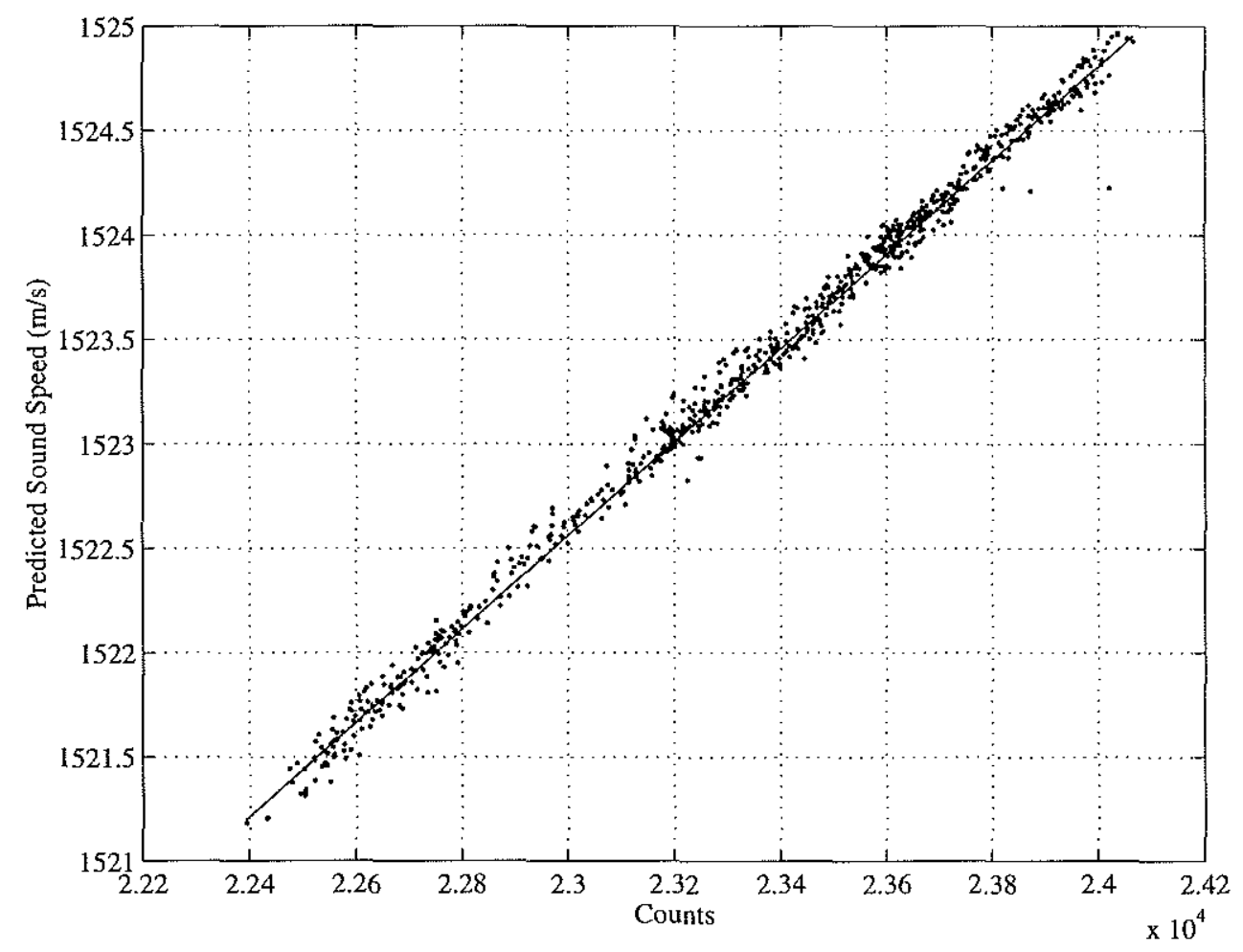

Figure 2.4: Sound speed calibration curve for one sensor from the Hudmix deployment. The solid line is the best-fitting curve.

where $c$ is speed of sound in $\mathrm{m} / \mathrm{s}, T$ is temperature in degrees $\mathrm{C}, s$ is salinity in ppt and $D$ is pressure in m. As Figure 2.4 indicates, BASS accurately tracked the variation in predicted sound speed, $\left(r^{2}>0.99\right.$ for four out five pods, with one pod showing signs of persistent malfunction). The only shortcoming was that the sound speed in (2.5) exhibited a rectified dependence on $v_{p}$ (i.e. $c=2 c_{0}-\frac{c_{0}^{2}}{d} t_{1}-\left|v_{p}\right|$ ), and therefore would not cancel if both normal and reverse measurements were averaged.

Although the absolute travel-time meter appeared to work reliably in a mean sense, individual bursts were dominated by instrument noise (Figure 2.5, top). Stacking and averaging of a number of bursts indicated that a similar low-frequency oscillation occurred in 
each burst (Figure 2.6). The oscillation caused an apparent velocity fluctuation of about $1 \mathrm{~m} / \mathrm{s}$ and was assumed to be a resonance in the BASS electronics. In addition, the high frequency fluctuations (magnitude $\approx 0.5 \mathrm{~m} / \mathrm{s}$ ) superimposed on the oscillation were almost and order of magnitude larger than sound speed changes caused by measured temperature fluctuations predicted by (2.6). The high-frequency fluctuations were considered instrument noise. Figure 2.7 is a comparison of measured sound speed spectra and a theoretical spectrum estimated using Kolmogorov's theory of the inertial subrange. The noise floor of the measured spectrum clearly overwhelms the energy level of the predicted spectrum. These problems prohibit estimation of temperature flux and temperature dissipation with the original design of the instrument.

\subsection{Instrument Modifications}

We were motivated to improve BASS's absolute travel-time electronics by encouraging results of the mean sound speed analysis which indicated that eventual success in resolving fluctuating sound speeds in possible. Specifically, we set out to correct the following problems uncovered in the analysis of the Hudmix data: (1) water velocity rectification, (2) large electronic oscillation, and (3) high noise floor. An initial laboratory test of the original circuit confirmed that the oscillation and noise were caused by the BASS electronics rather than extraordinary features of the flow during Hudmix (Figure 2.5, middle and Figure 2.7).

The dependence of sound speed on flow velocity was rectified in the absolute travel-time measurement because the integrating capacitor was not stopped until both-received. Regardless of flow direction, the capacitor charged until the slower pulse arrived. This problem was 

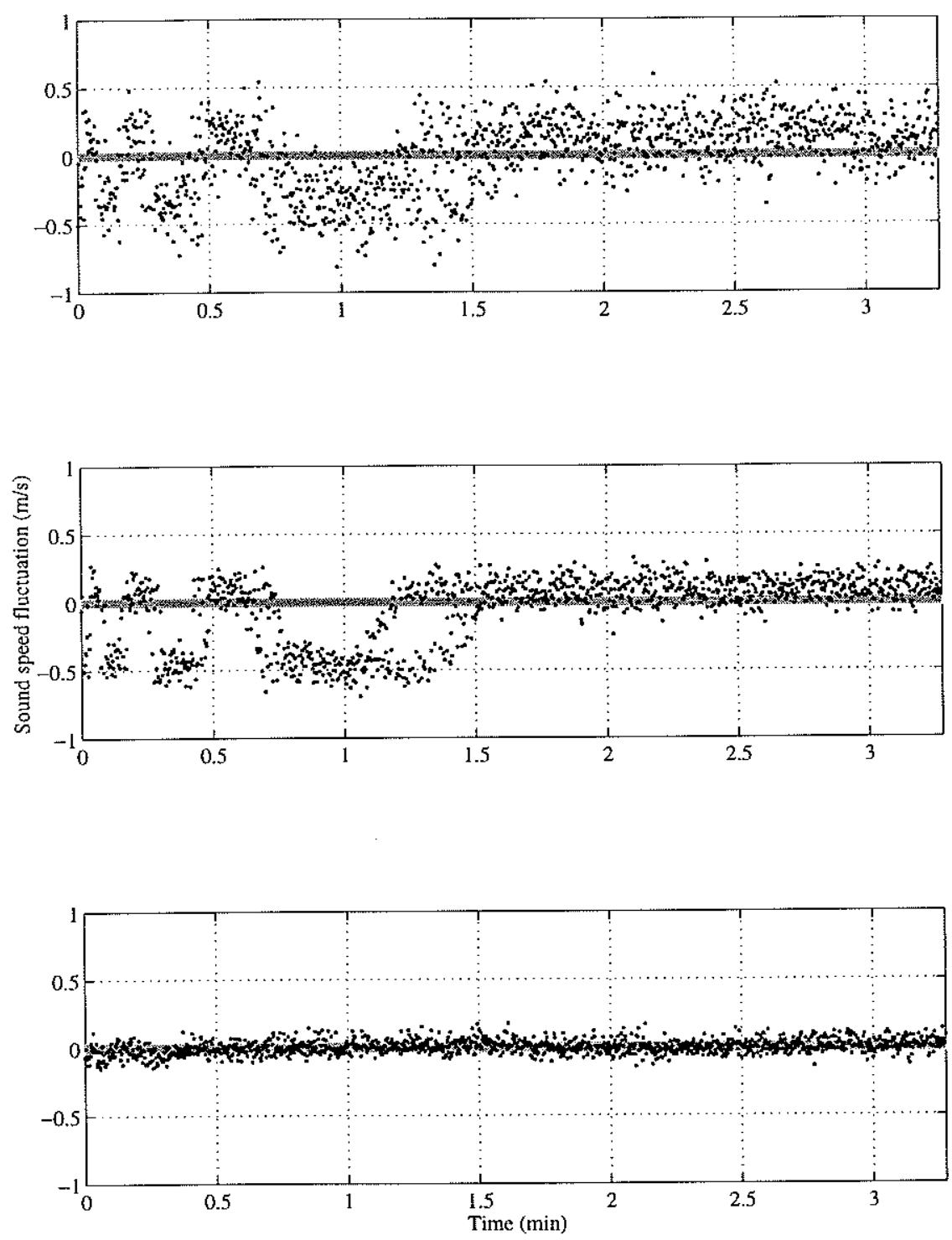

Figure 2.5: Examples of burst absolute travel-time time series. Top, Hudmix data. Middle, lab test of the original design. Bottom, lab test of modified design. For comparison, the magnitude of signal caused by the observed temperature fluctuations (from thermistors) during the Hudmix deployment is indicated by the gray region. During Hudmix, the standard deviation deviation of the temperature fluctuations was $20 \mathrm{mC}$, corresponding to sound speed fluctuations of $75 \mathrm{~mm} / \mathrm{s}$. 


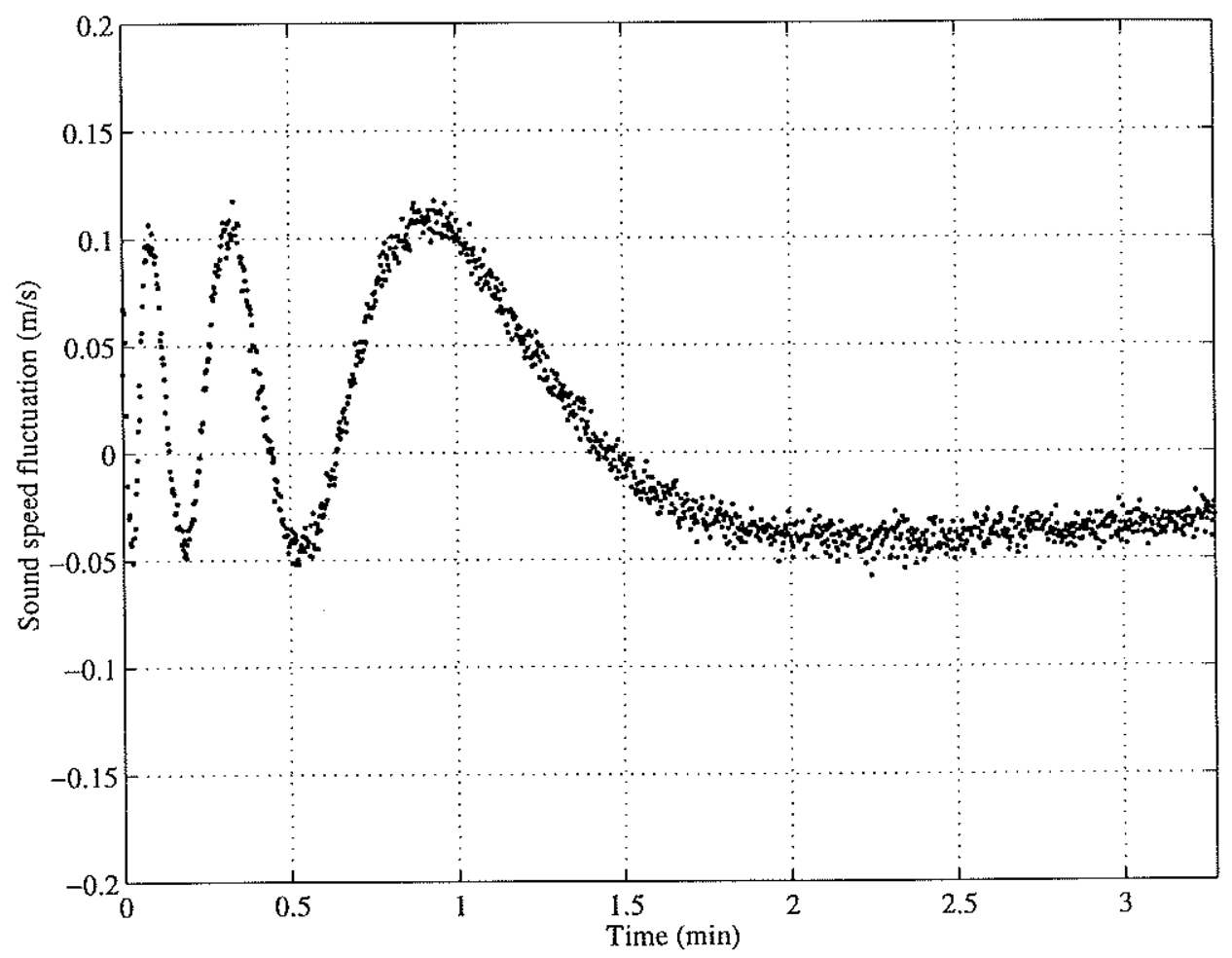

Figure 2.6: Averages of 36 absolute travel-time bursts from the Hudmix deployment illustrating the consistent oscillations within the bursts. 


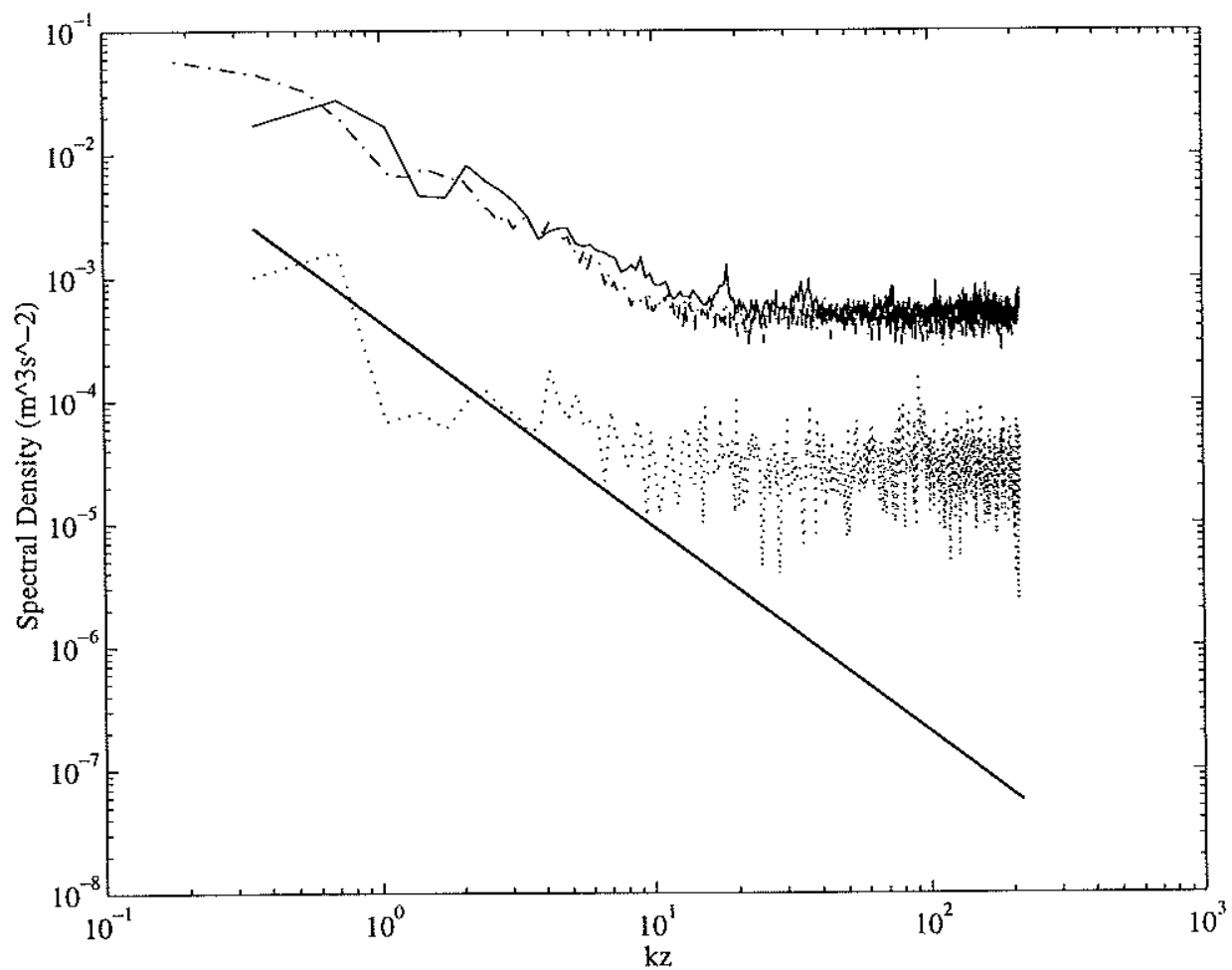

Figure 2.7: Sound speed spectra. The solid bold line is a theoretical prediction based on Kolmogorov's theory of the inertial subrange and conditions typical of the Hudson estuary. Solid, dash and dash-dot lines correspond to measured spectra from the Hudson, from a lab test of the original design and from a lab test of the modified design, respectively. Temporal data are converted to spatial data using Taylor's frozen turbulence hypothesis. 
corrected by stopping the integration when trigger circuit A (Figure 2.2, bottom) detected on arrival, 14 th-received-A. For a normal measurement, $t_{1}$ is measured. When the switching circuit reversed a short time later, $t_{2}$ is measured. These two measurements can then be averaged (Equation 2.2) to eliminate the component of travel-time due to flow velocity.

The oscillations observed in the Hudmix data were caused by the point in time at which integration was started (Figure 2.2 and Figure 2.3, top). Integration began at unclamp, a point fixed relative to start-transmit. If the frequency of the pulse varies, an error occurs because the integrator is started relative to the start of the transmission rather than the 14th negative-going zero-crossing of the transmission. Apparently, the oscillator that generates the acoustic pulse has a temperature dependent frequency. The hear generated by resistive losses in the electronics is a source of repeatable temperature variations. This heat causes the temperature to rise at the start of each burst but cools between bursts. We significantly reduced the magnitude of the oscillation by measuring the time between start-transmit and 14 th-transmitted.

Our strategy to lower the noise level of the absolute travel-time measurement was to reduce integration noise by reducing the integration period. The original design took advantage of the accuracy of BASS's electronic clock by starting integration $85 \mu$ s into the timing period, which left about $15 \mu$ s to be integrated over until the pulse arrived. To take advantage of the electronics clock and still measure relative to the correct phase of the pulse, we used two integrating circuits in our modified design (Figure 2.3, bottom). The first integrating circuit measures the time between start-transmit and 14th-transmitted. The second integrating circuit measures the time between unclamp and 14 th-received- $A$. In addition, unclamp was moved forward in time by $6.5 \mu \mathrm{s}$. 
Figure 2.8 is a simplified schematic diagram of the modified design. The two integrating circuits receive independent start and stop times. High impedance voltage followers track the voltage on the integrating capacitors and drive a difference amplifier with a gain of 8 . This modified design is a significant improvement over the original design. The electronic oscillation has been almost completely removed and noise level has been reduced by a factor of five from the Hudmix measurements of $0.1 \mathrm{~m} / \mathrm{s}$, to about the level of the signal predicted by the Hudmix temperature data (Figure 2.5, bottom). The noise floor of the spectra measured with the modified design has been lowered by more than an order of magnitude (Figure 2.7).

Presently, the predicted level of the Hudson Estuary temperature inertial subrange is still obscured by measurement errors (Figure 2.7). However, simple statistical arguments shown that the white noise will not affect the correlation between temperature and velocity in $\mathrm{f}$ the data record is of sufficient length. Therefore, heat and buoyancy fluxes may be estimated with the modified circuit design.

\subsection{Conclusions}

The analysis of absolute travel-time data recorded in the Hudson estuary and subsequent modifications of the absolute travel-time circuit used in BASS suggest the following conclusions:

(1) During the Hudmix deployment, mean sound speed measured by BASS agreed remarkable well with the sound speeds predicted by measured temperature, salinity, and pressure. In a mean sense, BASS is capable of measuring the sound speed in coastal ocean 


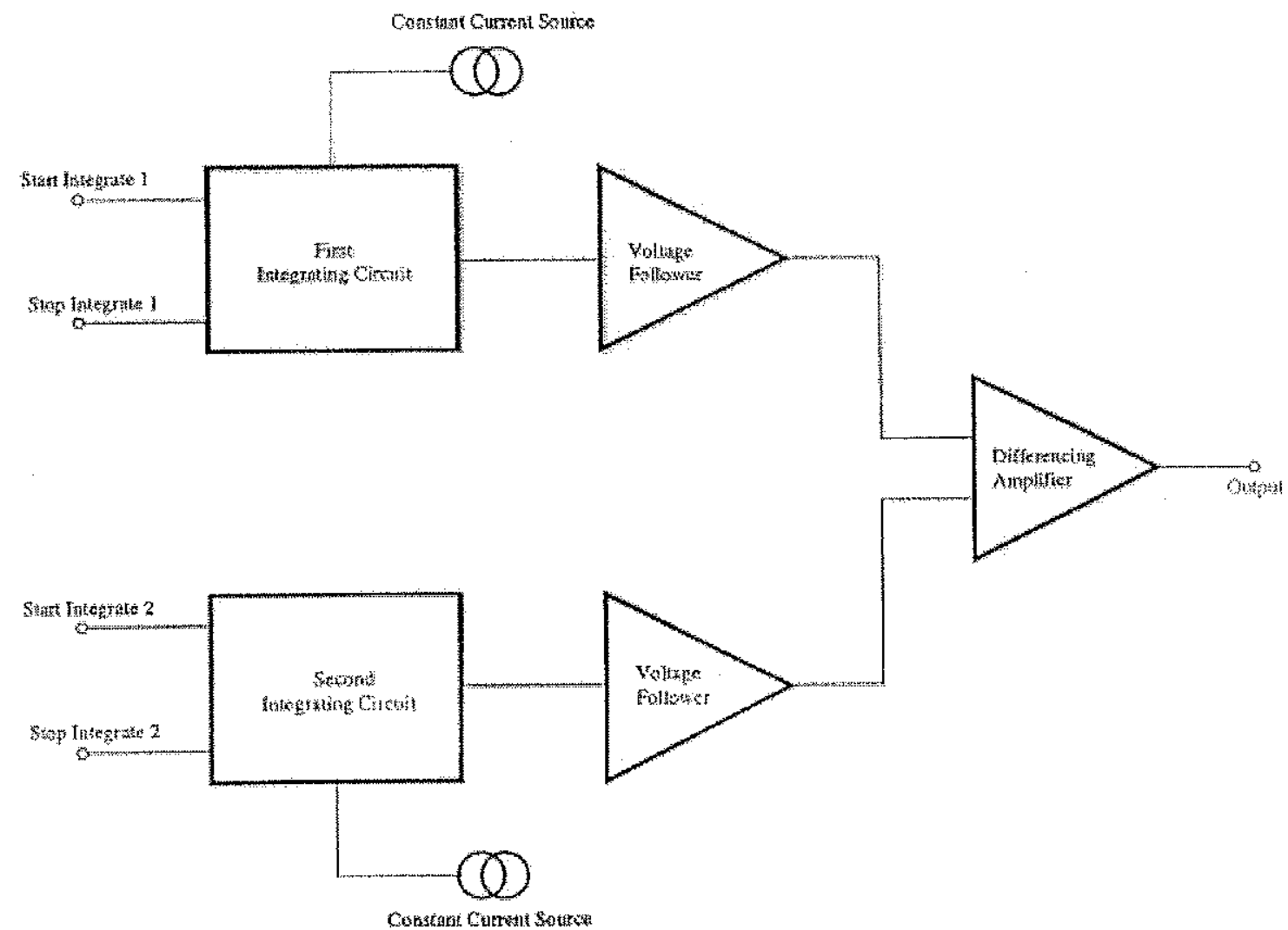

Figure 2.8: Simplified schematic diagram of modified absolute travel-time circuit. 
environments.

(2) During the Hudmix deployment, several problems were identified in the fluctuating sound speed measurements recorded by BASS. The most serious were an electronic oscillation and a high noise floor. The absolute travel-time circuit was modified to minimize these problems.

(3) Presently, the electronic oscillation has been almost completely removed and the noise floor has been reduced by more than an order of magnitude. The magnitude of the oscillation and noise must be further reduced to resolve the predicted level of the Hudson Estuary temperature inertial subrange. However, simple statistical arguments show that records of sufficient length can lead to robust estimates of hear and buoyancy fluxes.

\section{ACKNOWLEDGEMENTS}

This work was supported by ONR grant number N000149510373. We benefited from discussions with Todd Morrison. This is WHOI contribution number 9288. 


\section{Bibliography}

K. V. MacKenzie. Nine-term equation for sound speed in the oceans. J. Acoust. Soc. Am., $70: 807-812,1981$.

A. S. Monin and A. M. Yaglom. Statistical Fluid Mecahnics. MIT Press, Cambridge, Mass., 1971.

D. A. Trivett. Diffuse Flow from Hydrothermal Vents. PhD thesis, WHOI/MIT, 1991.

A. J. Williams, J. S. Tochko, R. L. Koehler, W. D. Grant, Gross T. F., and C. V. R Dunn. Measurement of turbulence in the oceanic bottom boundary layer with an acoustic current meter array. J. Atm. and Ocean. Tech., 4:312-327, 1987. 


\title{
Chapter 3
}

\section{The Direct Estimation of Near-bottom Turbulent Fluxes in the Presence of Energetic Wave Motions}

\author{
William J. Shaw and John H. Trowbridge
}

(This chapter represents a manucript that is to be submitted to The Journal of Atmospheric and Oceanic Technology.)

\begin{abstract}
Velocities produced by energetic waves can contaminate direct covariance estimates of near-bottom turbulent shear stress and turbulent heat flux. A new adaptive filtering technique is introduced to minimize the contribution of wave-induced motions to measured covariances. The technique requires the use of two sensors separated in space and assumes that the spatial coherence scale of the waves is much longer than the spatial coherence scale of the turbulence. The proposed technique is applied to an extensive set of data collected in the bottom boundary layer of the New England Shelf. Results from the oceanic test indicate that the technique succeeds at removing surface wave contamination from shear stress and heat flux estimates using pairs of sensors separated in the vertical dimension by a distance of approximately five times the height of the lower sensor, even during the close passage of hurricanes. However, the technique fails at removing contamination caused by internal waves which occur occasionally in the data set. The internal wave case is complicated by the facts that the internal waves are highly intermittent, that the internal wave period is comparable to the Reynolds-averaging period, that the height of the internal wave boundary layer is of the order of the height of measurement and, specifically for heat flux estimates, that nonlinear effects are large. The presence of internal waves does not pose a significant problem for estimating turbulent shear stress because contamination caused by internal waves is limited to frequencies lower then the stress carrying eddies. In contrast, the
\end{abstract}


presence of internal waves does pose a problem for estimating turbulent heat flux because the contamination extends in the range of the heat flux carrying eddies.

\subsection{Introduction}

Turbulent mixing driven by bottom drag is an essential component of shallow water flows. The dynamically significant effects of turbulence on Reynolds-averaged boundary layer flows are contained in the covariance terms that represent the vertical transport of heat and horizontal momentum [e.g., Monin and Yaglom, 1971]. As a result, the measurement of near-bottom, turbulence-induced shear stress and heat flux is a critical objective of coastal physical oceanography. High-quality vertical flux estimates are necessary to formulate and test hypotheses regarding the dynamics of boundary layer turbulence. For example, models of currents and sediment transport on continental shelves rely heavily on how turbulent momentum flux is parameterized in the bottom boundary layer [e.g., Grant and Madsen, 1986].

A number of methods are available for estimating turbulent fluxes in oceanic boundary layers. A direct vertical flux estimate is obtained by measuring the covariance between turbulent fluctuations of the transported quantity of interest (horizontal momentum or temperature) and fluctuations of vertical velocity [e.g., Bowden and Fairbairn, 1956,

Heathershaw, 1979]. Indirect methods include the profile technique [e.g., Charnock, 1959, Sternberg, 1968, Gross and Nowell, 1983, Lueck and Lu, 1997], in which fluxes are determined from the fit of semi-empirical models to the vertical profiles of Reynolds-averaged quantities; the inertial-dissipation technique [e.g., Gross and Nowell, 1985, Green and McCave, 1995], in which fluxes are derived from Kolmogorov's theory of the inertial subrange 
and a simplified variance budgets; and the dynamic technique [e.g., Bowden and Fairbairn, 1952, Bowden et al., 1959], in which fluxes are estimated as the residual after other terms in simplified momentum or heat balances are measured. Both the profile technique and the inertial-dissipation technique rely on restrictive assumptions that are often hard to justify in the coastal ocean because of the presence of multiple length scales. In practice, the dynamic technique measures the influence of turbulence on a broader scale than the other techniques and, additionally, the researcher usually desires to test the balances instead of assuming that they hold. For example, an important question is whether form drag is a significant component of the bottom resistance felt by the overlying flow [McLean and Smith, 1979]. Because of the complexity of the coastal bottom boundary layer, we believe that direct measurements of turbulent fluxes are required to advance our understanding of its dynamics.

In the coastal ocean, however, direct turbulent flux measurements are often contaminated by the presence of energetic surface waves [Grant et al., 1984, Grant and Madsen, 1986, Huntley and Hazen, 1988, Trowbridge, 1998] and the presence of internal waves can have a similar effect. Wave contamination of flux estimates occurs if the principal axes of the wave-induced velocity field, which do not necessarily coincide with the measurement coordinates and may be influenced by physical processes such as bottom topography or rotational motions, are not known with sufficient accuracy. Because surface waves and internal waves are often more energetic than turbulent eddies by several orders of magnitude, a slight uncertainty in estimating the principal axes of the wave-induced velocity field can easily swamp the desired turbulence-induced component of measured covariance.

Recently, Trowbridge [1998] introduced a novel technique for removing surface wave 
contamination from shear stress estimates by differencing records of velocity components measured by pairs of spatially separated current meters. The technique is based on the assumption that the spatial scale of surface waves is long in comparison with the correlation scale of the near-bottom turbulence. Essentially, Trowbridge assumed that that the waveinduced velocities at the two sensors are equal and can be canceled by subtraction.

Here, alternative differencing strategies are considered and a new adaptive filtering technique is introduced to further reduce wave contamination of measured covariances. The adaptive filtering technique represents a generalization of Trowbridge's differencing technique in that the basic assumption concerning the wave motions is made less stringent by allowing the wave motions at two locations separated in space to be coherent rather than equal. The extended technique allows useful flux estimates to be made in conditions of higher wave energy and admits a wider latitude in the placement of sensors. Importantly, the technique can be applied to a vertical array of sensors, which is the most practical configuration for field experiments.

In this paper, we consider the effects of surface and internal waves on estimates of the vertical turbulent fluxes of heat and horizontal momentum. We limit the scope to nearbottom flows and to methods for removing wave-induced contributions to measured fluxes. We present the differencing and filtering techniques for removing wave bias from turbulent flux estimates and we present observational results that show how well the techniques work in practice. The remainder of the paper is organized into the following sections: a theoretical description of the wave bias problem and a presentation of techniques for removing wave bias (Section 3.2), a description of a set of measurements used to test the proposed techniques (Section 3.3), a presentation and discussion of the results (Sections 5.4 and 5.5), and a 
summary and conclusions (Section 5.6).

\subsection{Theoretical Framework}

\subsubsection{Overview}

A two-dimensional (2-D) model of the velocity field of the bottom boundary layer on the continental shelf in the presence of wave motions is developed to demonstrate the wave bias problem and to provide a framework for the presentation of techniques for removing wave bias. Although the general problem is three-dimensional (3-D), a 2-D analysis in which the direction of the desired turbulent shear stress, the wave motions, and the components of the instrument coordinate system lie in a plane, captures the important features of the wave bias problem in a simple manner. The analysis presented here is a simplification of that presented by Trowbridge [1998] in that it is $2-\mathrm{D}$, although it has been expanded to include the effects of waves on heat flux estimates and it relaxes assumptions about near-equality of wave-induced motions at the spatial scales of interest.

The analysis relies on several assumptions. The most fundamental assumption is that the ratio of the spatial coherence scale of the wave-induced fluctuations to the spatial coherence scale of the turbulence-induced fluctuations is large, so that a spatial separation exists at which the wave-induced fluctuations are coherent and the turbulence-induced fluctuations are incoherent. Also, we require that the wave- and turbulence-induced fluctuations are incoherent with one another and that the statistical properties of the waves and turbulence are stationary. For the purpose of illustration, we assume that the velocity sensors have perfect response, that all components of the turbulent covariance tensor have equal 
magnitudes (which is a good assumption for boundary layer flows [e.g., Tennekes and Lumley, 1972]), that the waves are small-amplitude and narrow-banded in frequency, and that stratification is due to a uniform vertical temperature gradient.

The model (Figure 3.1 ) has a position vector, $\mathbf{x}=[x, z]$. The horizontal component lies in the common direction of the wave propagation and the desired shear stress and the vertical component is defined positive upwards with $z=0$ at the bed. In the model, the velocity vector $\mathbf{u}=[u, w]$ is composed of contributions from mean current, waves, and turbulence: $\mathbf{u}=\overrightarrow{\mathbf{u}}+\tilde{\mathbf{u}}+\mathbf{u}^{\prime}$, where an overbar represents a mean value and tilde and prime denote wave and turbulent fluctuations, respectively. Similarly, the temperature is denoted by $T=\bar{T}+\tilde{T}+T^{\prime}$. The wave-induced component of the fluctuations can include contributions due to both surface and internal waves, although we are not concerned here with any possible interactions between the two. The model quantities of interest with this notation, the turbulent shear stress and turbulent vertical heat flux, are $-\overline{\rho u^{\prime} w^{\prime}}$ and $\rho c_{p} \overline{T^{\prime} w^{\prime}}$, respectively, where $\rho$ is density and $c_{p}$ is heat capacity.

An estimate of $\overline{u^{\prime} w^{\prime}}$ using the velocity $\mathbf{U}=[U, W]$ measured in instrument coordinates that are rotated a small angle, $\theta$, from the model coordinate system (Figure 3.1 ) to first order in $\theta$ is

$$
\operatorname{cov}(U, W)=\overline{u^{\prime} w^{\prime}}+\overline{\tilde{u} \tilde{w}}+\theta\left[\left(\overline{w^{\prime 2}}-\overline{u^{\prime 2}}\right)+\left(\overline{\tilde{w}^{2}}-\overline{\tilde{u}^{2}}\right)\right]
$$

Likewise, an estimate of $\overline{T^{\prime} w^{\prime}}$ to first order in $\theta$ is

$$
\operatorname{cov}(T, W)=\overline{T^{\prime} w^{\prime}}+\overline{\tilde{T} \tilde{w}}+\theta\left(\overline{T^{\prime} u^{\prime}}+\overline{\tilde{T} \tilde{u}}\right)
$$

The first term on the right side of (3.1) and (3.2) is the desired vertical turbulent flux of 


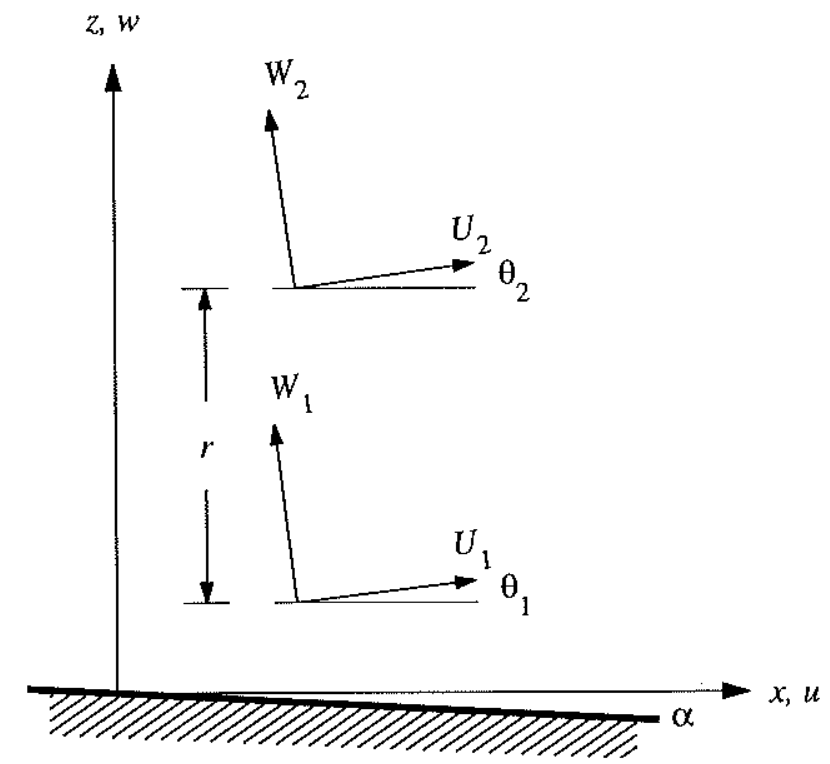

Figure 3.1: Schematic diagram illustrating the geometry of the 2-D bottom boundary layer model. The boundary layer is measured with two instruments whose velocity components $\left(U_{1}, W_{1}\right)$ and $\left(U_{2}, W_{2}\right)$ are oriented at small angles $\theta_{1}$ and $\theta_{2}$, respectively, to the model coordinate system $(u, w)$. The sea bed slopes at a small angle, $\alpha$ 
horizontal momentum or heat, respectively. The second term is a 'real' wave bias, representing vertical transport by waves. The third and fourth terms are 'apparent' turbulenceand wave- induced biases arising from the orientation of the instrument coordinate system. The 'apparent' turbulence bias is the well-known instrument levelling error [Pond, 1968].

For the case of gentle bottom slopes, small rotation angles, and irrotational waves, Trowbridge [1998] demonstrated that the turbulence bias in (3.1) is small but that the wave biases can be an order of magnitude larger than the desired turbulent momentum flux. Observations in the atmospheric boundary layer indicate that the components of the heat flux vector have the same order of magnitude [Kaimal et al., 1972], indicating that the turbulence bias in (3.2) is small compared to the desired covariance if $\theta$ is small. We can estimate the 'real' wave bias in (3.2) as follows. For the case of a uniform vertical temperature gradient and small-amplitude motions, the wave-induced temperature fluctuations arise from advection of the vertical temperature gradient and, for progressive waves, may be estimated as

$$
\tilde{T}=-z \frac{d \bar{T}}{d z} \frac{\tilde{u}}{c}
$$

where $c$ is the wave phase speed. $\overline{T^{\prime} w^{\prime}}$ may be roughly estimated as $-\kappa z u_{*} d \bar{T} / d z$ [e.g. Businger et al., 1971], where $u_{*}$ is the bottom friction velocity and $\kappa(=0.4)$ is von Karman's constant. With these estimates, the ratio of wave-induced bias to turbulent heat flux is

$$
\frac{\overline{\tilde{T} \tilde{w}}}{\overline{\overline{T^{\prime} w^{\prime}}}}=O\left(\frac{\theta \overline{\tilde{T} \tilde{u}}}{\overline{\overline{T^{\prime} w^{\prime}}}}\right)=O\left(\frac{\theta \overline{\tilde{u}^{2}}}{u_{*} c}\right)
$$

indicating that for near-bottom values typical of the outer shelf for surface waves $\left(\overline{\tilde{u}^{2}}=\right.$ $0.01 \mathrm{~m}^{2} \mathrm{~s}^{-2}, c=20 \mathrm{~m} / \mathrm{s}, u_{*}=0.01 \mathrm{~m} / \mathrm{s}$, and $\theta=0.01$ ) the wave-induced bias is three orders 
of magnitude smaller than the turbulent heat flux.

If the measured velocity vector can be rotated into the principal axes of the waveinduced velocity field, the wave biases are removed. In practice, one is unlikely to know the orientation of the principle coordinates of the wave-induced velocity field to sufficient accuracy to remove wave bias by rotation. The objective of the present analysis is thus to consider alternative techniques for removing the 'real' and 'apparent' wave biases from estimates of the turbulent covariances $\overline{u^{\prime} w^{\prime}}$ and $\overline{T^{\prime} w^{\prime}}$ in (3.1) and (3.2).

\subsubsection{Differencing Strategies}

Trowbridge [1998] showed that one can reduce the wave bias in estimates of momentum flux to an acceptable level by differencing measurements obtained from two sensors separated by a distance large in comparison to the correlation scale of the turbulence but small in comparison to the inverse wavenumber of the waves, with the critical assumptions that the correlation scale of the waves is much greater than the correlation scale of the turbulence and that the waves and turbulence are uncorrelated. Here, we rely on the same basic concept and assumptions, but consider a slightly different approach in which only one of the measurements comprising the covariance is differenced. This approach is beneficial because the resulting covariance estimate corresponds to the position of a single sensor, rather than an average of the covariances at each sensor, and two estimates are available for each sensor. The resulting turbulence estimates are not independent, but the waves are removed with essentially independent information, so that the redundant estimates provide a check on the technique.

In the following, a parenthesized subscript is used to identify the position where a 
particular measurement is obtained. For example, $u_{(1)}$ is the horizontal velocity measured at the location of the first sensor. The operation of differencing between two sensors is represented by an upper-case delta sign and the operation of averaging between two sensors is represented by angular brackets. For example, $\Delta u$ and $\langle u\rangle$ are the difference and average, respectively, of the horizontal velocities measured by a pair of sensors. We focus here on the differencing of velocity measurements.

We consider three estimates of $\overline{u^{\prime} w^{\prime}}$ given measurements at a pair of sensors: $\frac{1}{2} \operatorname{cov}(\Delta U, \Delta W)$, $\operatorname{cov}\left(\Delta U, W_{(1)}\right)$, and $\operatorname{cov}\left(U_{(1)}, \Delta W\right)$. With the assumptions that wave- and turbulenceinduced velocities are uncorrelated and that the turbulence is uncorrelated at the two locations, the three estimates of $\overline{u^{\prime} w^{\prime}}$ can be written to first order in $\theta$

$$
\begin{aligned}
\frac{1}{2} \operatorname{cov}(\Delta U, \Delta W) & =\left\langle\overline{u^{\prime} w^{\prime}}\right\rangle+\left\langle\theta\left(\overline{w^{\prime 2}}-\overline{u^{\prime 2}}\right)\right\rangle+\frac{1}{2} \operatorname{cov}(\Delta \tilde{U}, \Delta \tilde{W}) \\
\operatorname{cov}\left(\Delta U, W_{(1)}\right) & =\overline{u_{(1)}^{\prime} w_{(1)}^{\prime}}+\theta_{(1)}\left(\overline{w_{(1)}^{\prime 2}}-\overline{u_{(1)}^{\prime 2}}\right)+\operatorname{cov}\left(\Delta \tilde{U}, \tilde{W}_{1}\right)
\end{aligned}
$$

and

$$
\operatorname{cov}\left(U_{(1)}, \Delta W\right)=\overline{u_{(1)}^{\prime} w_{(1)}^{\prime}}+\theta_{(1)}\left(\overline{w_{(1)}^{\prime 2}}-\overline{u_{(1)}^{\prime 2}}\right)+\operatorname{cov}\left(\tilde{U}_{1}, \Delta \tilde{W}\right)
$$

The first term on the right side of (3.5) is an average of the turbulence-induced covariance at each of the sensors and the first term on the right side of (3.6) and (3.7) is the desired turbulence-induced covariance at the location of the first sensor. The second term on the right side of (3.5)-(3.7) is a small 'apparent' turbulence bias. The wave component of the 
three estimates may be written to first order in $\theta$

$$
\begin{aligned}
\frac{1}{2} \operatorname{cov}(\Delta \tilde{U}, \Delta \tilde{W}) & =\frac{1}{2} \overline{\Delta \tilde{u} \Delta \tilde{w}}+\frac{1}{2}\langle\theta\rangle\left(\overline{\Delta \tilde{w}^{2}}-\overline{\Delta \tilde{u}^{2}}\right)+\frac{1}{4} \Delta \theta\left(\Delta \overline{\tilde{w}^{2}}-\Delta \overline{\tilde{u}^{2}}\right) \\
\operatorname{cov}\left(\Delta \tilde{U}, \tilde{W}_{(1)}\right) & =\overline{\tilde{w}_{(1)} \Delta \tilde{u}}+\theta_{(1)}\left(\overline{\tilde{w}_{(1)} \Delta \tilde{w}}-\overline{\tilde{u}_{(1)} \Delta \tilde{u}}\right)+\Delta \theta\left(\overline{\tilde{w}_{(1)}^{2}}-\overline{\tilde{w}_{(1)} \Delta \tilde{w}}\right),
\end{aligned}
$$

and

$$
\operatorname{cov}\left(\tilde{U}_{(1)}, \Delta \tilde{W}\right)=\overline{\tilde{u}_{(1)} \Delta \tilde{w}}+\theta_{(1)}\left(\overline{\tilde{w}_{(1)} \Delta \tilde{w}}-\overline{\tilde{u}_{(1)} \Delta \tilde{u}}\right)-\Delta \theta\left(\overline{\tilde{u}_{(1)}^{2}}-\overline{\tilde{u}_{(1)} \Delta \tilde{u}}\right) \cdot(3
$$

Wave contributions to the measured covariance arise from differences in wave-induced velocity between the two locations and differences in alignment between the two sensors.

At this point, we introduce approximations in an effort to illustrate the magnitudes of the terms in (3.8)-(3.10), restricting the scope of the analysis to the measurement of turbulence in the presence of surface waves with a pair of vertically separated sensors. Differenced quantities are represented with first-order expansions. For example, $\Delta u \simeq r \partial \tilde{u} / \partial z$, where $r$ is the separation between the sensors and $\partial \tilde{u} / \partial z$ is understood to be evaluated at a location between the two sensors for the estimate $\frac{1}{2} \operatorname{cov}(\Delta \tilde{U}, \Delta \tilde{W})$ and at the location of the first sensor for the estimates $\operatorname{cov}\left(\Delta U, W_{(1)}\right)$ and $\operatorname{cov}\left(U_{(1)}, \Delta W\right)$. Also, we assume that $k z \ll 1$, where $k$ is the wavenumber of the waves, which allows the hyperbolic functions describing the vertical variation of wave-induced velocity to be represented with first-order expansions. With these assumptions, (3.8)-(3.10) can be approximated as

$$
\frac{1}{2} \operatorname{cov}(\Delta \tilde{U}, \Delta \tilde{W}) \simeq \frac{r^{2}}{2 z^{2}}\left[k^{2} z^{2}\langle\overline{\tilde{u} \tilde{w}}\rangle+\langle\theta\rangle\left(\overline{\left\langle\tilde{w}^{2}\right\rangle}-k^{4} z^{4} \overline{\left\langle\tilde{u}^{2}\right\rangle}\right)+\Delta \theta\left(\overline{\left\langle\tilde{w}^{2}\right\rangle}-k^{4} z^{4}\langle\overline{\bar{u}} \overline{2}(3)]\right] 1\right)
$$




$$
\operatorname{cov}\left(\Delta \tilde{U}, \tilde{W}_{(1)}\right) \simeq \frac{r}{z}\left[k^{2} z^{2} \overline{\tilde{u}_{(1)} \tilde{w}_{(1)}}+\theta_{(1)}\left(\overline{\tilde{w}_{(1)}^{2}}-k^{2} z^{2} \overline{\tilde{u}_{(1)}^{2}}\right)+\Delta \theta \overline{\tilde{w}_{(1)}^{2}}\left(\frac{z}{r}+1\right)\right]
$$

and

$$
\operatorname{cov}\left(\tilde{U}_{(1)}, \Delta \tilde{W}\right) \simeq \frac{r}{z}\left[\overline{\tilde{u}_{(1)} \tilde{w}_{(1)}}+\theta_{(1)}\left(\overline{\tilde{w}_{(1)}^{2}}-k^{2} z^{2} \overline{\tilde{u}_{(1)}^{2}}\right)-\Delta \theta \overline{\tilde{u}_{(1)}^{2}}\left(k^{2} z^{2}+\frac{z}{r}\right)\right]
$$

For near-bottom measurements, we expect that terms like $\overline{\tilde{u} \tilde{w}}, \theta \overline{\tilde{u}^{2}}$, and $\Delta \theta \overline{\tilde{u}^{2}}$ are of comparable magnitude and much larger than terms like $\theta \overline{\tilde{w}^{2}}$ and $\Delta \theta \overline{\tilde{w}^{2}}$. All of the large terms on the right side of (3.11) and (3.12) have been reduced by at least the factor $k^{2} r z$, while two of the large terms in (3.13) have only been reduced by the factor $r / z$, which is constrained by the assumptions of the technique to be of order one, as seen below. It is obvious that the stress estimate $\frac{1}{2} \operatorname{cov}(\Delta \tilde{U}, \Delta \tilde{W})$ has approximately a factor of two reduction in wave bias at the cost of averaging the turbulent covariances between the two locations. Note that for near-bottom measurements, the 'real' wave bias is roughly equal in the estimates $\frac{1}{2} \operatorname{cov}(\Delta \tilde{U}, \Delta \tilde{W})$ and $\operatorname{cov}\left(\Delta \tilde{U}, \tilde{W}_{(1)}\right)$ while the reduction in 'apparent' wave bias resulting from the rotation of horizontal velocity into the vertical is better for $\frac{1}{2} \operatorname{cov}(\Delta \tilde{U}, \Delta \tilde{W})$ by an additional factor of $k^{2} r z$.

In order for these differencing techniques to work in practice, the sensor separation must be made large relative to the correlation length scale of the turbulence so that turbulence cross-correlation terms between the sensors that were neglected in (3.5) - (3.7), which are $\overline{u_{(1)}^{\prime} u_{(2)}^{\prime}}, \overline{u_{(1)}^{\prime} w_{(2)}^{\prime}}, \overline{w_{(1)}^{\prime} u_{(2)}^{\prime}}$, are small. It is easily shown that all of the cross correlation terms except $\overline{u_{(1)}^{\prime} w_{(2)}^{\prime}}$ are multiplied by $\theta$, and therefore with the assumption that the elements of the turbulent correlation function tensor have the same order of magnitude, we only 
need to ensure that $\overline{u_{(1)}^{\prime} w_{(2)}^{\prime}}$ is small in comparison with $\overline{u_{(1)}^{\prime} w_{(1)}^{\prime}}$. We have established an empirical guideline for meeting this condition by considering the off-diagonal component of the turbulent correlation function tensor obtained in an estuarine bottom boundary layer. Trowbridge et al. [in press] reported the deployment in August 1995 of a quadrapod on the bed of the Hudson River adjacent to Manhattan, New York, for two weeks with a vertical array of five BASS current meters (see Section 3.3) at heights of $0.3,0.6,1.2,2.1$, and $2.4 \mathrm{~m}$ above the bottom in a wave-free turbulent boundary layer. The instruments were sampled in bursts of 3.28 minutes at $6.25 \mathrm{~Hz}$. To evaluate the importance of $\overline{u_{(1)}^{\prime} w_{(2)}^{\prime}}$, we calculated representative cross-correlation functions $R_{u w}=\overline{u(z) w(z+r)}$ by taking the average of $R_{u w}$ from individual bursts over a period of five days (Figure 3.2). The cross-correlation functions indicate that the ratio of the vertical separation to the height of the lower sensor $r / z$ must be greater than approximately five for the turbulence to be considered uncorrelated, i.e. less than one-tenth of the value at zero separation. In this particular environment, the cross-correlation function decreases more rapidly as $z$ increases, possibly because the scale of the eddies is limited by factors other than height above bottom [Trowbridge et al., in press].

\subsubsection{Further Reduction in Wave Bias with Adaptive Filtering}

Failure of the differencing technique described in Section (3.2.2) occurs under conditions of high wave energy if differences in amplitude or phase of the wave-induced motions between the two locations cause the difference terms $\Delta \tilde{u}$ or $\Delta \tilde{w}$ appearing in (3.8)-(3.10) to be significant. When this problem occurs, a solution is to minimize the difference terms with linear filtration techniques. Assuming that the wave-induced fluctuations are completely spatially 


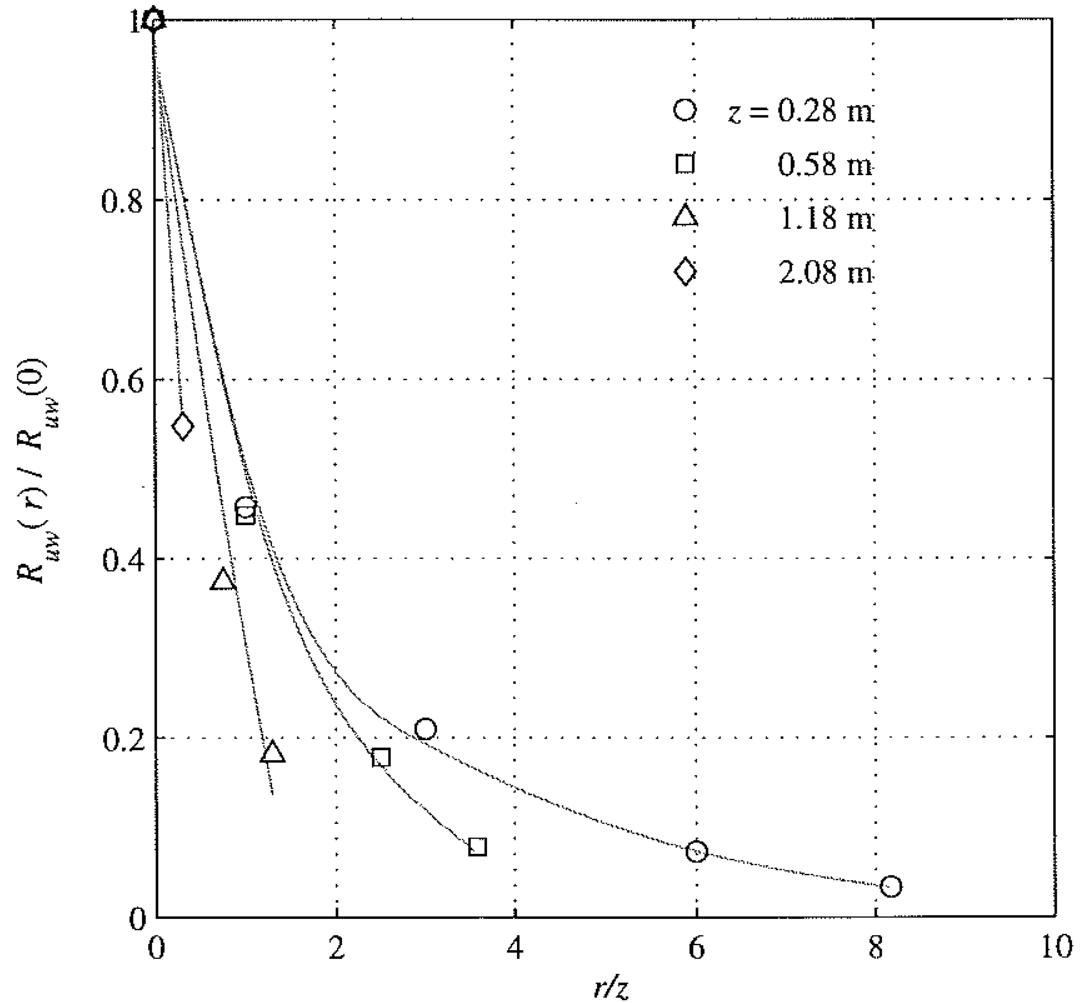

Figure 3.2: Estimates of the normalized cross-correlation function $R_{u w}(r) / R_{u w}(0)$, where $R_{u w}(r)=\overline{u(z) w(z+r)}$, for positive vertical separations $r$ estimated from measurements obtained in the Hudson River Estuary at four heights above the bottom. Each curve represents an average derived from 7203.28 minute bursts of data. 
coherent, least-squares filtering can be used to estimate the coherent component of velocity at one position with velocity measurements at the second position. Thus, the assumption behind the simple differencing technique used earlier, that the wave-induced motions at the two sensors are essentially identical, is replaced by the assumption that the wave-induced motions at the two sensors are essentially perfectly coherent. The assumption that the turbulence is spatially uncorrelated is replaced by the assumption that the turbulence is spatially incoherent.

Here, the analysis is no longer limited to vertical separations. We begin by using the assumption that the wave motions at the two positions are coherent to establish a linear functional relationship between the wave-induced fluctuations measured at position (1) and wave-induced fluctuations measured at position (2). For example, the wave-induced horizontal velocities are assumed to be related by

$$
\tilde{U}_{(1)}(t)=\int_{-\infty}^{\infty} h\left(t^{\prime}\right) \tilde{U}_{(2)}\left(t-t^{\prime}\right) d t^{\prime}
$$

Here, $t$ is time and $h(t)$ is a filter that represents the spatial transfer function between the wave-induced fluctuations at the two locations. In effect, (3.14) states that if $\tilde{U}_{(1)}$ and $\tilde{U}_{(2)}$ are perfectly coherent, then $\tilde{U}_{(1)}$ is completely predictable from $\tilde{U}_{(2)}$. The goal of this analysis, then, is to use the total measured velocities to estimate the transfer functions as well as possible. A more nearly wave-free estimate of horizontal velocity at position (1) than $\Delta U=U_{(1)}-U_{(2)}$ is then $\Delta \hat{U}=U_{(1)}-\hat{U}_{(1)}$ where

$$
\hat{U}_{(1)}(t)=\int_{-\infty}^{\infty} \hat{h}\left(t^{\prime}\right) U_{(2)}\left(t-t^{\prime}\right) d t^{\prime}
$$


is the estimated wave-induced velocity at position (1) using estimates of the transfer function, $\hat{h}(t)$, and the measured velocities at position (2). The estimated wave velocity $\hat{U}_{(1)}$ contains a turbulence component, but it is of no consequence so long as the assumption that the turbulence is spatially incoherent is valid. If the estimates cov $\left(\Delta U, W_{(1)}\right)$ and $\operatorname{cov}\left(U_{(1)}, \Delta W\right)$ are replaced by $\operatorname{cov}\left(\Delta \hat{U}, W_{(1)}\right)$ and $\operatorname{cov}\left(U_{(1)}, \Delta \hat{W}\right)$, respectively, the problematic difference terms appearing in (3.9) and (3.10) are minimized to the extent that the model assumptions are valid.

The filter weights in (3.14) are estimated by finding the ordinary least-squares solution of a transversal filter model [e.g., Haykin, 1996] that has has been modified to a non-causal form for our post-processing purposes:

$$
\mathbf{A h}=\mathbf{U}_{(1)}
$$

Here, $\mathbf{A}$ is an $M \times N$ windowed data matrix of velocity at position (2), where $M$ is the number of data points and $N$ is the number of filter weights ( $N$ must be odd for the filter to be symmetric), $\mathbf{h}$ is a vector of filter weights, and $\mathbf{U}_{(1)}$ is a vector of position (1) velocity. The $m$-th row of $\mathrm{A}$ is $\left[u\left(i-\frac{N-1}{2}\right), \ldots, u(i), \ldots, u\left(i+\frac{N-1}{2}\right)\right]$, in which the notation $u(i)$ is temporarily understood to mean the $i$-th discrete sample of $u$. The solution is

$$
\hat{\mathbf{h}}=\left(\mathbf{A}^{T} \mathbf{A}\right)^{-1} \mathbf{A}^{T} \mathbf{U}_{(1)}
$$

and estimates of the wave-induced velocity at position (1), $\hat{\mathbf{U}}_{(1)}$ are found by convolving 
the measured velocity record with the estimated filter weights

$$
\hat{\mathbf{U}}_{(1)}=\mathbf{A} \hat{\mathbf{h}} .
$$

In practice, we have encountered some complications in applying the filtering technique described above (to be discussed below), but, in general, we have found that with the adjustment of only two significant parameters, the number of data points $M$ and the number of filter weights $N$, robust estimates of the wave spatial transfer functions are obtainable.

\subsection{Measurements and Analysis}

The techniques described in Section 3.2 are tested on a set of measurements collected as part of the Coastal Mixing and Optics (CMO) experiment during 1996-97. One component of the field experiment consisted of the deployment of a bottom tripod equipped with current meters in order to study the turbulence dynamics of the coastal bottom boundary layer. The techniques are applied to vertically separated pairs roughly satisfying the empirical constraints discussed in Section 3.2.2. Three sets of turbulent shear stress and vertical heat flux estimates were made: raw covariance, covariance with differencing, and covariance with differencing and adaptive filtering.

For the purposes of this paper, we limit our attention to a six-week long deployment of the bottom tripod which began August 17, 1996. The tripod was deployed at a depth of approximately $70 \mathrm{~m}$ on the New England Shelf. The 'SuperBASS' tripod deployed during the CMO experiment [Fredericks et al., 1999] was outfitted with a vertical array of BASS current meters [Williams et al., 1987]. The BASS sensor measures the three-dimensional 
velocity vector by determining the differential travel-time of acoustic pulses traveling in opposite direction along four $15-\mathrm{cm}$ acoustic axes. The BASS electronics were recently modified to measure the absolute travel-time of acoustic pulses [Trivett, 1991, Shaw et al., 1996], allowing the measurement of sound speed in addition to fluid velocity. The vertical BASS array consisted of seven sensors at heights of $0.4,0.7,1.1,2.2,3.3,5.4$, and $7.0 \mathrm{~m}$ above the bed. The sensors were sampled at $1.2 \mathrm{~Hz}$ in 27 minute bursts for a total of 2060 samples per burst. The bursts were taken in two-hour cycles consisting of 3 half-hour periods during which the instruments were sampled followed by one half-hour period during which the instruments were idle.

Velocity and sound speed measurements from the BASS array were used to produce estimates of turbulent shear stress and vertical heat flux at the lower six measurement levels. For the purposes of this paper, sound speed fluctuations were converted to temperature fluctuations by assuming the existence of a linear relationship with a constant estimated as $\partial T / \partial C=0.5 \mathrm{deg} \mathrm{C} / \mathrm{m} / \mathrm{s}$ from the equation of state [MacKenzie, 1981] at a reference salinity of $32 \mathrm{psu}$, which is the average salinity during the first tripod deployment. All flux estimates were derived from individual $27 \mathrm{~min}$ bursts of $1.2 \mathrm{~Hz}$ data. Approximately satisfying the empirical constraint $r>5 z$, the sensors were paired as follows for the purposes of differencing: $0.38 \mathrm{~m}$ and $2.20 \mathrm{~m}, 0.74 \mathrm{~m}$ and $3.30 \mathrm{~m}$, and $1.10 \mathrm{~m}$ and $5.40 \mathrm{~m}$.

In order to test the techniques described in Section 3.2 , we computed three sets of $\overline{u^{\prime} w^{\prime}}$ estimates in an order of increasing sophistication to remove wave contamination: a 'raw' estimate, $\operatorname{cov}(U, W)$, two 'differenced' estimates, $\operatorname{cov}(\Delta U, W)$ and $\operatorname{cov}(U, \Delta W)$, and two 'filtered' estimates, $\operatorname{cov}(\Delta \hat{U}, W)$ and $\operatorname{cov}(U, \Delta \hat{W})$. An analogous set of $\overline{T^{\prime} w^{\prime}}$ covariances were also computed. Flux estimates were obtained by integrating the cospectrum of $u(t)$ 
and $w(t)$, denoted $C o_{u w}$, and the cospectrum of $T(t)$ and $w(t)$, denoted $C o_{T w}$. All spectral and cospectral calculations were carried out in a coordinate system aligned with the burstaveraged flow and resulting shear stress estimates were then rotated into a coordinate system oriented in the along- and cross-shelf directions.

We obtained estimated wave-induced velocity records with an expanded form of the transversal filter model (3.16) in which all three components of velocity measured at the second sensor were used as inputs, resulting in a $N \times 3 M$ data matrix $\mathbf{A}$. We chose to include all three velocity components in $\mathbf{A}$ because doing so increases the rank of $\mathbf{A}$ by approximately a factor of three whilie have an insignificant effect on the number of degrees of freedom of the regression so long as $M \gg N$. Filtered temperature records were obtained by applying the original model (3.16) directly, with $T$ replacing $U$. The filter weights were calculated with (3.17) and applied to the measured data according to (3.18). The length of the filter was $N=11$, corresponding to a window length of $9.2 \mathrm{~s}$, which is about half of the typical wave period at the CMO site, and resulted in a total of 33 filter weights for each estimated component of wave-induced velocity fluctuations and 11 filter weights for estimated wave-induced temperature fluctuations. New filters were calculated for each burst, so that the filtering technique was block adaptive to slowly changing wave conditions. As a result, there were $M-3 N=2027$ degrees of freedom in the velocity regression and $M-N=2049$ degrees of freedom in the temperature regression. 


\subsection{Results}

\subsubsection{Conditions at the CMO Site}

Before proceeding to a test of the proposed techniques for removing wave bias, it is worthwhile to briefly describe the conditions at the CMO field site during the first deployment. Here, we describe the temporal variability of burst statistics and we present spectra representative of conditions of energetic surface waves and conditions of energetic internal waves.

Time series of near-bottom mean currents at the CMO site (Figure 3.3a) are usually dominated by a rotary semi-diurnal tide with an amplitude of approximately $0.1 \mathrm{~m} / \mathrm{s}$. Subtidal events consist of predominantly westward flows with velocities up to $0.2 \mathrm{~m} / \mathrm{s}$. Time series of mean sound speed measured by the top and bottom BASS sensors (Figure 3.3b) indicate that the bottom boundary layer at the CMO site is usually well-mixed in sound speed. Occasionally, however, near-bottom stratification is strong, resulting in sound speed differences between 0.38 and $5.50 \mathrm{~m}$ above the bed of up to $10 \mathrm{~m} / \mathrm{s}$, which roughly corresponds to $5 \mathrm{degC}$. Time series of the standard deviation of horizontal velocity, $\sqrt{\sigma_{u}^{2}+\sigma_{v}^{2}}$ (Figure 3.3c), contain contributions from surface waves, from internal waves, and, to a much lesser extent, from turbulence. Surface waves generated by the close passage of Hurricane Edouard, a Nor'easter, and Hurricane Hortense are visible as peaks in $\sqrt{\sigma_{u}^{2}+\sigma_{v}^{2}}$ centered on days 246,251 , and 258 , respectively. The intermittent peaks of short duration are associated with internal waves with periods of the order of 5-15 minutes, as determined from the inspection of individual burst time series (an example of which is presented below).

Representative spectra of longitudinal and vertical velocity fluctuations, $S_{u u}$ and $S_{w w}$, during conditions of strong surface waves (Figure 3.4a) contain an energetic surface wave 

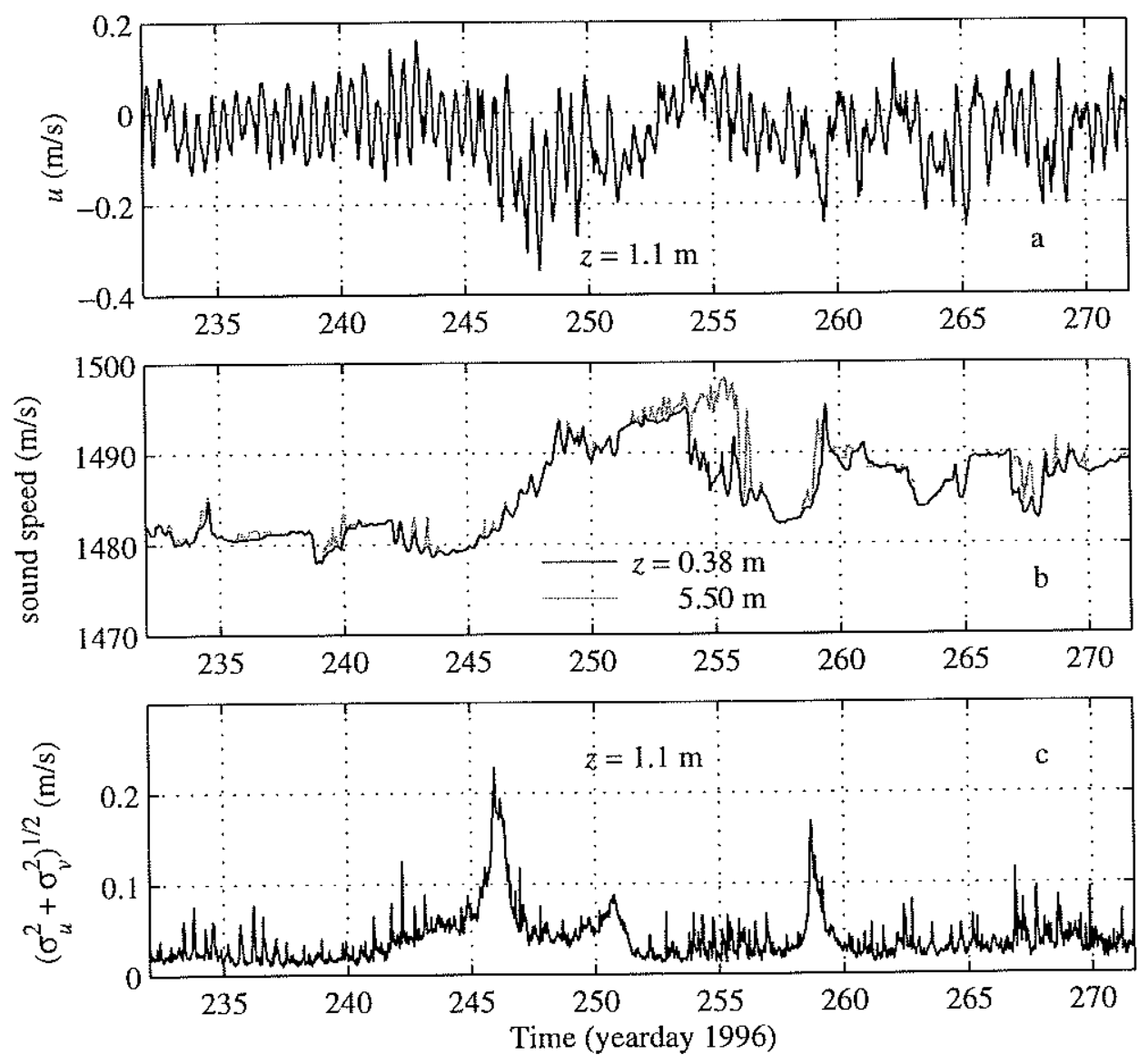

Figure 3.3: Time series of burst-averaged along-shelf current (a), burst-averaged sound speed (b), and burst standard deviations of horizontal velocity, $\sqrt{\sigma_{u}^{2}+\sigma_{v}^{2}}$, (c) measured by the BASS array during the first deployment of the CMO experiment. Statistics are from 27 minute bursts. The coordinate system is oriented so that eastward flow is positive. 
peak centered at $0.07 \mathrm{~Hz}$, corresponding to a period of $15 \mathrm{~s}$, a wavelength greater than 300 $\mathrm{m}$, and a phase speed of $20 \mathrm{~m} / \mathrm{s}$. The spectral levels of the $S_{u u}$ wave peak are more than two orders of magnitude larger than the underlying stress-carrying eddies. The corresponding spectrum of temperature fluctuations, $S_{T T}$ (Figure $3.4 \mathrm{~b}$ ), contains a small peak at the dominant surface wave frequency, consistent with the notion that the ratio $\overline{\tilde{T}^{2}} / \overline{T^{2}}$ is small compared to the ratio $\overline{\tilde{u}^{2}} / \overline{u^{I 2}}$ that is embodied in the estimate (3.3).

Representative spectra of longitudinal and vertical velocity fluctuations during conditions of strong internal waves (Figure 3.5a) contain a low-frequency 'hump' in $S_{u u}$ below $0.002 \mathrm{~Hz}$. Above $0.002 \mathrm{~Hz}$, the spectral levels of the horizontal velocity fluctuations fall off rapidly, giving the spectrum a concave upward shape. For this case, $S_{T T}$ contains energetic low-frequency variability (Figure $3.5 \mathrm{~b}$ ), but in contrast to $S_{u u}$, the temperature spectral levels do not fall off rapidly above $0.002 \mathrm{~Hz}$.

\subsubsection{Shear Stress}

We begin by presenting an example of the estimated wave transfer functions between an elevation of $5.4 \mathrm{~m}$ and $1.1 \mathrm{~m}$ above the bed, positions (2) and (1), respectively, during energetic surface wave conditions (Figure 3.6) that demonstrates that the filters are physically meaningful. For clarity, we use a notation $h_{i j}$, where $i$ denotes the component of velocity that is the output of the filter and $j$ denotes the component of velocity that is the input to the filter. The estimation of $\tilde{U}_{(1)}$ is dominated by $h_{u u}$ (Figure 3.6a), which has a maximum at zero lag, as expected for vertically separated instruments, and decreases with increasing lag with a zero-crossing near the fourth lag, or about $3.3 \mathrm{~s}$, which is roughly consistent with a dominant surface wave period of $15 \mathrm{~s}$. The weights of $h_{u v}$ and $h_{u w}$ are near-zero, 

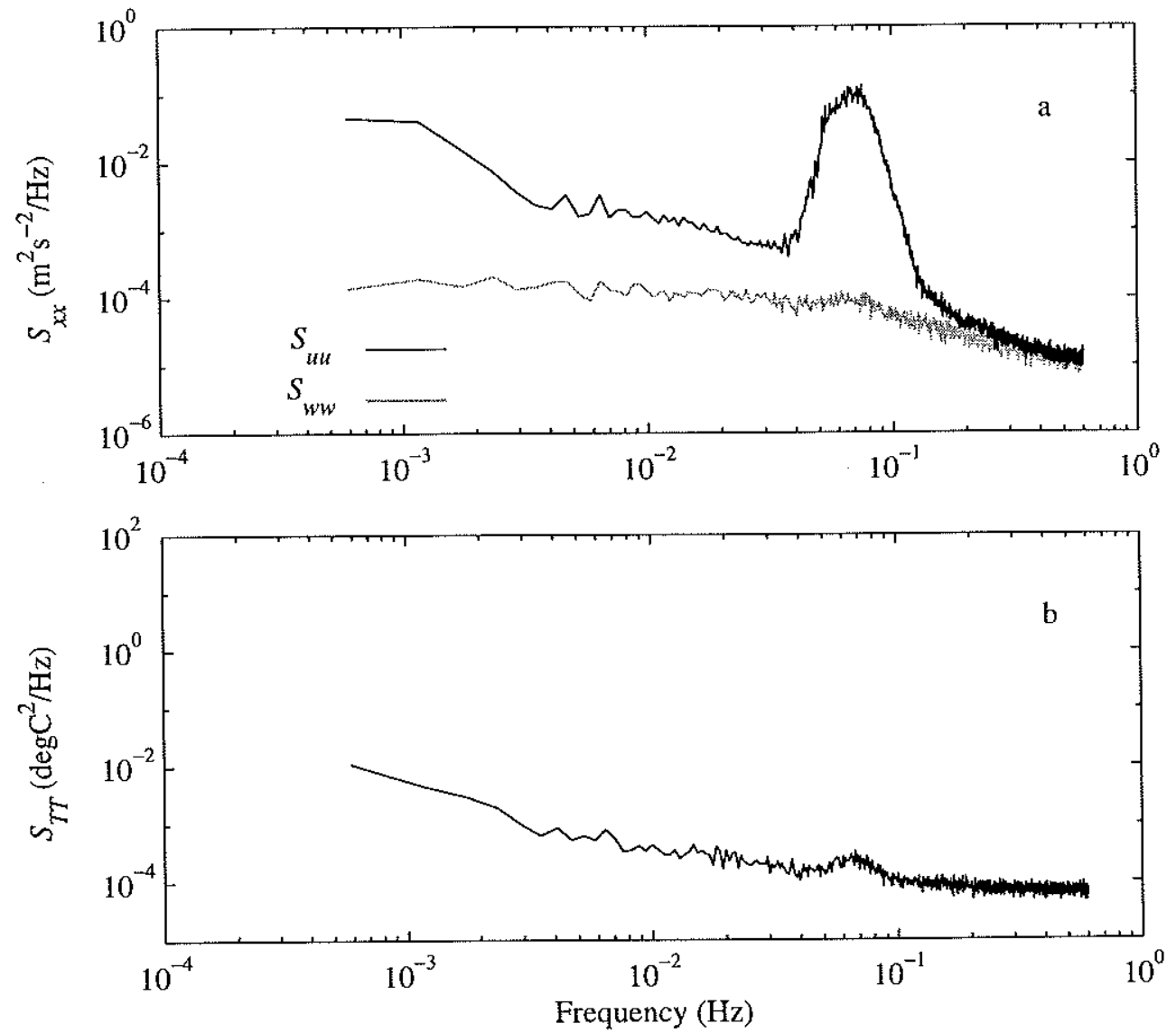

Figure 3.4: Averaged spectra of longitudinal and vertical velocity fluctuations (a) and temperature fluctuations (b) from BASS measurements at $0.70 \mathrm{~m}$ above the bed when energetic surface waves were present. The spectral densities plotted are average values from 49 bursts when the rms velocity in the surface wave peak was greater than $0.04 \mathrm{~m} / \mathrm{s}$. Rms surface wave velocity was estimated by integrating the area below the observed wave peak, $0.04 \mathrm{~Hz} \leq f \leq 0.2 \mathrm{~Hz}$, in the spectrum of horizontal velocity fluctuations. 

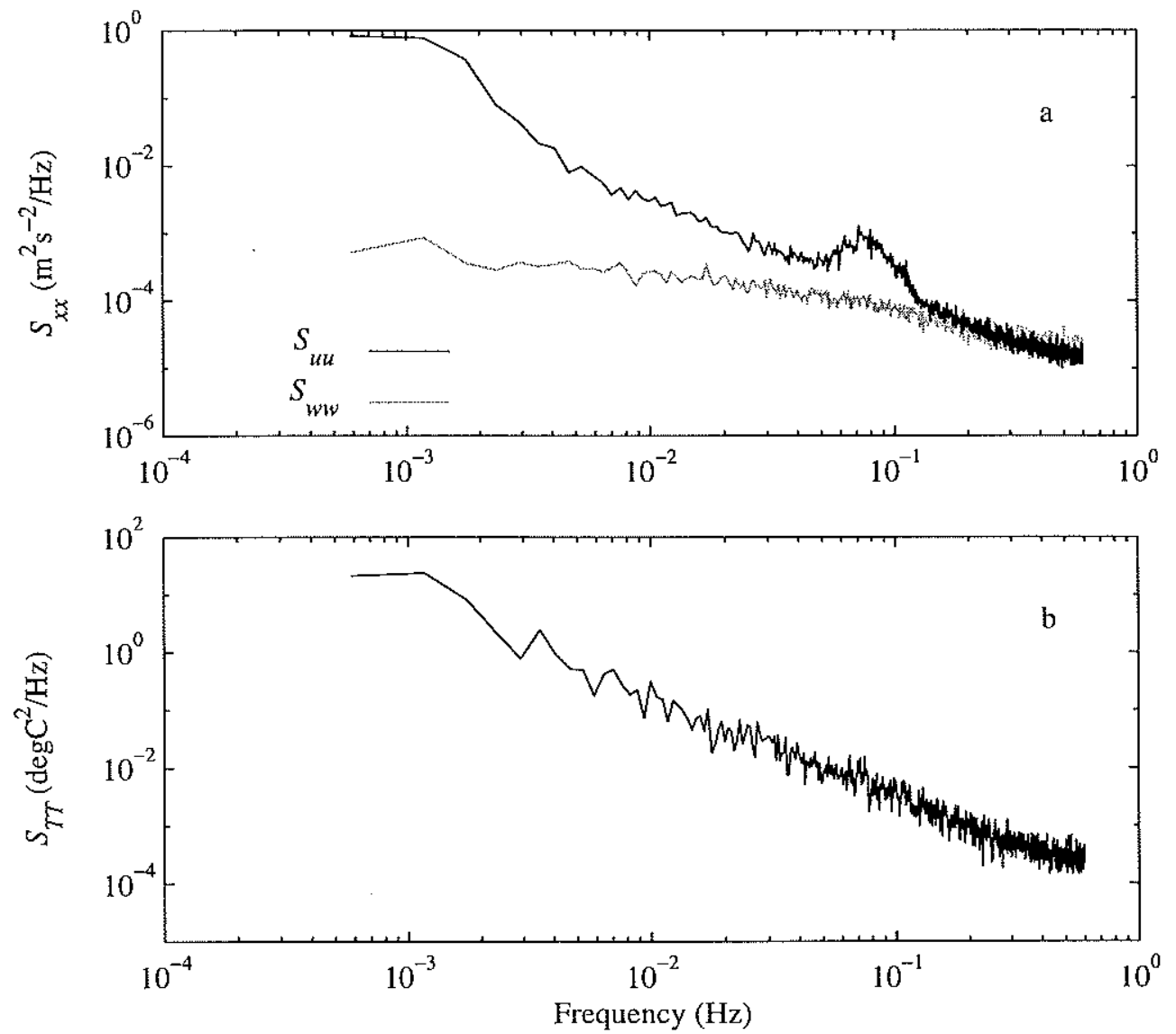

Figure 3.5: Average burst spectra of longitudinal and vertical velocity fluctuations (a) and temperature fluctuations (b) from BASS measurements at $0.70 \mathrm{~m}$ above the bed when energetic internal waves were present. The spectral densities plotted are average values from 49 bursts when the rms horizontal velocity minus the rms surface wave velocity was greater than $0.025 \mathrm{~m} / \mathrm{s}$. 
indicating that $V_{(2)}$ and $W_{(2)}$ are not important inputs in the estimation of $\tilde{U}_{(1)}$, although it is reassuring that they have been assigned near-zero values. In contrast, the estimation of $\tilde{W}_{(1)}$ is not completely dominated by $h_{w w}$ (Figure $3.6 \mathrm{~b}$ ), and the weights of $h_{u v}$ and $h_{u w}$ are significant with skew-symmetric peaks near the third lag, indicating that $U_{(2)}$ and $V_{(2)}$ are coherent with $\tilde{W}_{(1)}$ but are approximately in quadrature, as expected.

It is instructive to consider the cospectra that are integrated to yield the three sets of shear stress estimates described in Section 3.3, because wave-induced contributions can be recognized as deviations from the form expected in typical boundary layer flows. For reference, we present empirical forms for $C o_{u w}$ and the running integral of $C o_{u w}, \int_{0}^{f} C o_{u w}\left(f^{\prime}\right) d f^{\prime}$, which is known as an ogive curve (Figure 3.7a), that were determined from measurements in the wall region of the atmospheric boundary layer [Kaimal et al., 1972] and have been shown to be consistent with cospectra obtained in the wall region of shallow water, tidal bottom boundary layers [Soulsby, 1977]. The energetic eddies responsible for transmitting stress lie approximately in the range of apparent non-dimensional wavenumber $0.1<2 \pi f z / V<10$ with a peak near $2 \pi f z / V=1$, in which $V$ is the burst-averaged along-stream velocity and $2 \pi f / V$ is the apparent wavenumber of the turbulent eddies advected past a point sensor by $V$. When energetic waves are present, this form of Taylor's hypothesis is inadequate because advection of eddies by the wave motions is important [Lumley and Terray, 1983] and the actual wavenumber distribution is different than that given by $2 \pi f / V$. Because cospectral estimates are noisy, we choose to present the results in the form of ogive curves, which are essentially low-pass filtered cospectra, in which the features of interest are clearly observable.

As an example of the effect of moderately energetic surface waves on $C o_{u w}$, we present a 

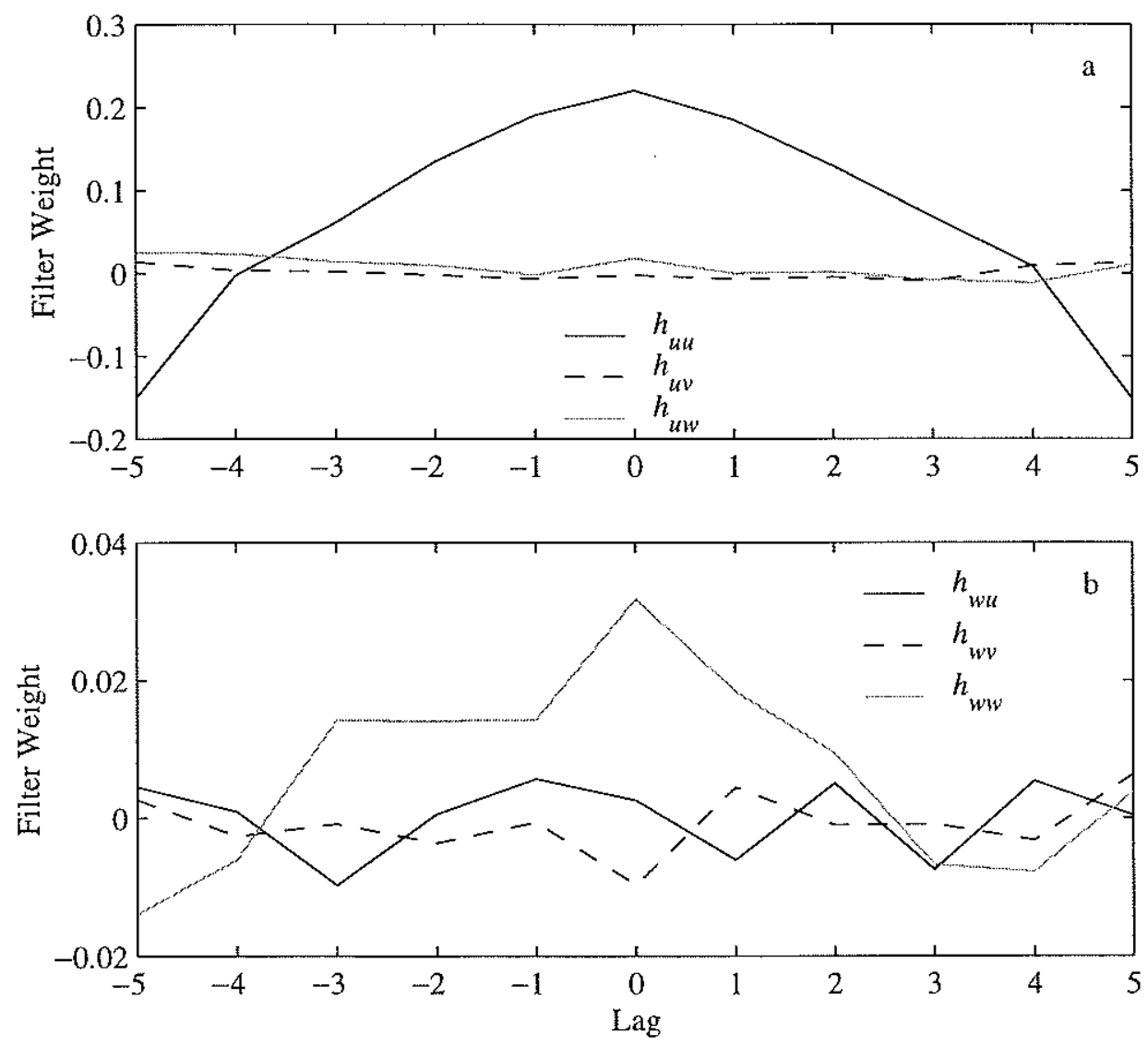

Figure 3.6: Example of wave transfer functions between an elevation of 5.4 and $1.1 \mathrm{~m}$ above the bottom. In panel (a), $h_{u u}, h_{u v}$, and $h_{u w}$ are the filter weights used to estimate $\tilde{U}_{(1)}$ given measurements of $U_{(2)}, V_{(2)}$, and $W_{(2)}$. In panel (b), $h_{w u}, h_{w v}$, and $h_{w w}$ are the filter weights used to estimate $\tilde{W}_{(1)}$ with the same measurements as in (a). The filter weights plotted are average values from 49 bursts when the rms surface wave velocity was greater than $0.04 \mathrm{~m} / \mathrm{s}$. 

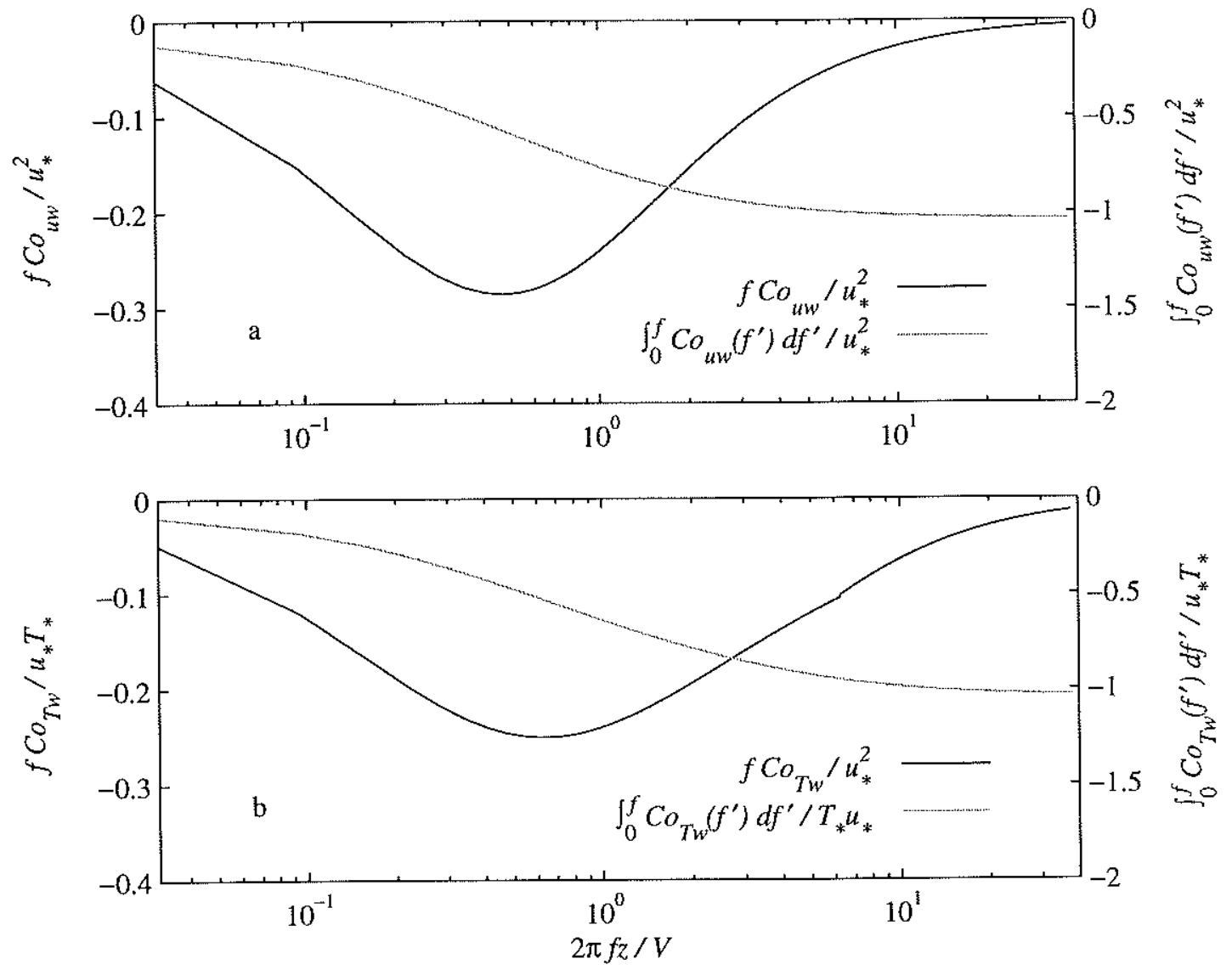

Figure 3.7: Empirical energy-preserving cospectrum and running integral of cospectrum of $u$ and $w(\mathrm{a})$ and of $T$ and $w(\mathrm{~b})$ as determined from measurements during neutral conditions in the atmospheric boundary layer [Kaimal et al., 1972]. The cospectra are normalized by $u_{*}=\left|\overline{u^{\prime} w^{\prime}}\right|^{1 / 2}$ and $T_{*}=-\overline{T^{\prime} w^{\prime}} / u_{*}$. 
spectrum of horizontal velocity and cospectral ogive curves at $0.70 \mathrm{~m}$ above the bed, position (1), for a burst in which the surface wave rms velocity equals $0.07 \mathrm{~m} / \mathrm{s}$ (Figure 3.8 ). The surface wave spectral peak (Figure 3.8a) is nearly collocated with the expected maximum in turbulence cospectral density (Figure 3.7a). The 'raw' covariance estimate has a large contribution at the frequency of the surface waves (Figure $3.8 \mathrm{~b}$ ) which is anomalous for boundary layer turbulence (Figure $3.7 \mathrm{a}$ ) and is attributable to wave contamination. Of the two 'differenced' estimates, $\operatorname{cov}\left(\Delta U, W_{(1)}\right)$ contains little or no surface wave contamination, while $\operatorname{cov}\left(U_{(1)}, \Delta W\right)$ contains a surface wave contribution of magnitude roughly equal to the 'raw' estimate (Figure 3.8c), which is consistent with expectations of Section 3.2.2. Neither of the 'filtered' estimates, $\operatorname{cov}\left(\Delta \hat{U}, W_{(1)}\right)$ or $\operatorname{cov}\left(U_{(1)}, \Delta \hat{W}\right)$, contains a net contribution to the shear stress from surface waves (Figure 3.8d). There is some contamination of $C o_{U \Delta \hat{W}}$; however, the filtering has compensated in a manner that yields no net contribution to $\operatorname{cov}\left(\Delta \hat{U}, W_{(1)}\right)$

As a further test of the techniques in removing surface wave contamination, we present an example of the effect of highly energetic surface waves on $C o_{u w}$, in which the surface wave $\mathrm{rms}$ velocity equals $0.12 \mathrm{~m} / \mathrm{s}$ at $0.70 \mathrm{~m}$ above the bottom (Figure 3.9 ). As in the example for moderate surface wave energy, the estimates $\operatorname{cov}\left(U_{(1)}, W_{(1)}\right)$ and $\operatorname{cov}\left(U_{(1)}, \Delta W\right)$ fail to remove wave contamination (Figure $3.9 \mathrm{~b}$ and $\mathrm{c}$ ) and the estimates $\operatorname{cov}\left(\Delta \hat{U}, W_{(1)}\right)$ and $\operatorname{cov}\left(U_{(1)}, \Delta \hat{W}\right)$ succeed at removing surface wave contamination (Figure $3.9 \mathrm{~d}$ ). In contrast, the 'differenced' estimate $\operatorname{cov}\left(\Delta U, W_{(1)}\right)$ also fails to remove surface wave contamination (Figure $3.9 \mathrm{c}$ ) indicating that the filtering technique is neccessary to remove wave contamination when highly energetic surface waves are present.

An example burst time series of longitudinal and vertical velocity, at $1.10 \mathrm{~m}$ above the 

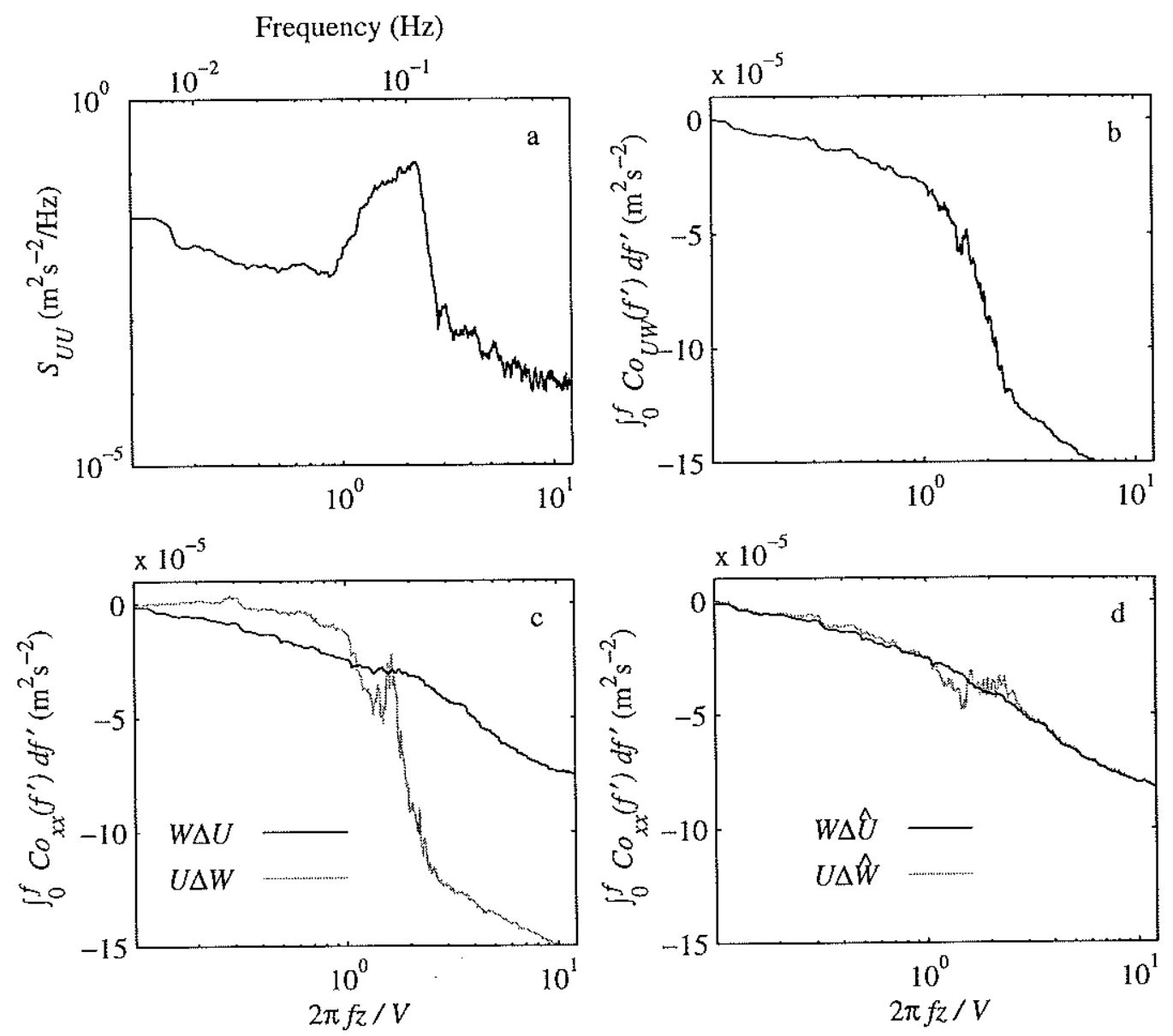

Figure 3.8: Estimates of $\overline{u^{\prime} w^{\prime}}$ under moderately energetic surface wave conditions (surface wave rms velocity $=0.07 \mathrm{~m} / \mathrm{s}$ ) from BASS measurements at $0.70 \mathrm{~m}$ above the bed taken on day 246 as the energy of the surface waves forced by Hurricane Edouard diminished. The spectrum of the longitudinal velocity fluctuations, smoothed with a 21-point boxcar filter, is given in panel (a). In panels (b-d) ogive curves for three sets of stress estimates are presented: 'raw' (b), 'differenced' (c) and 'filtered' (d). 

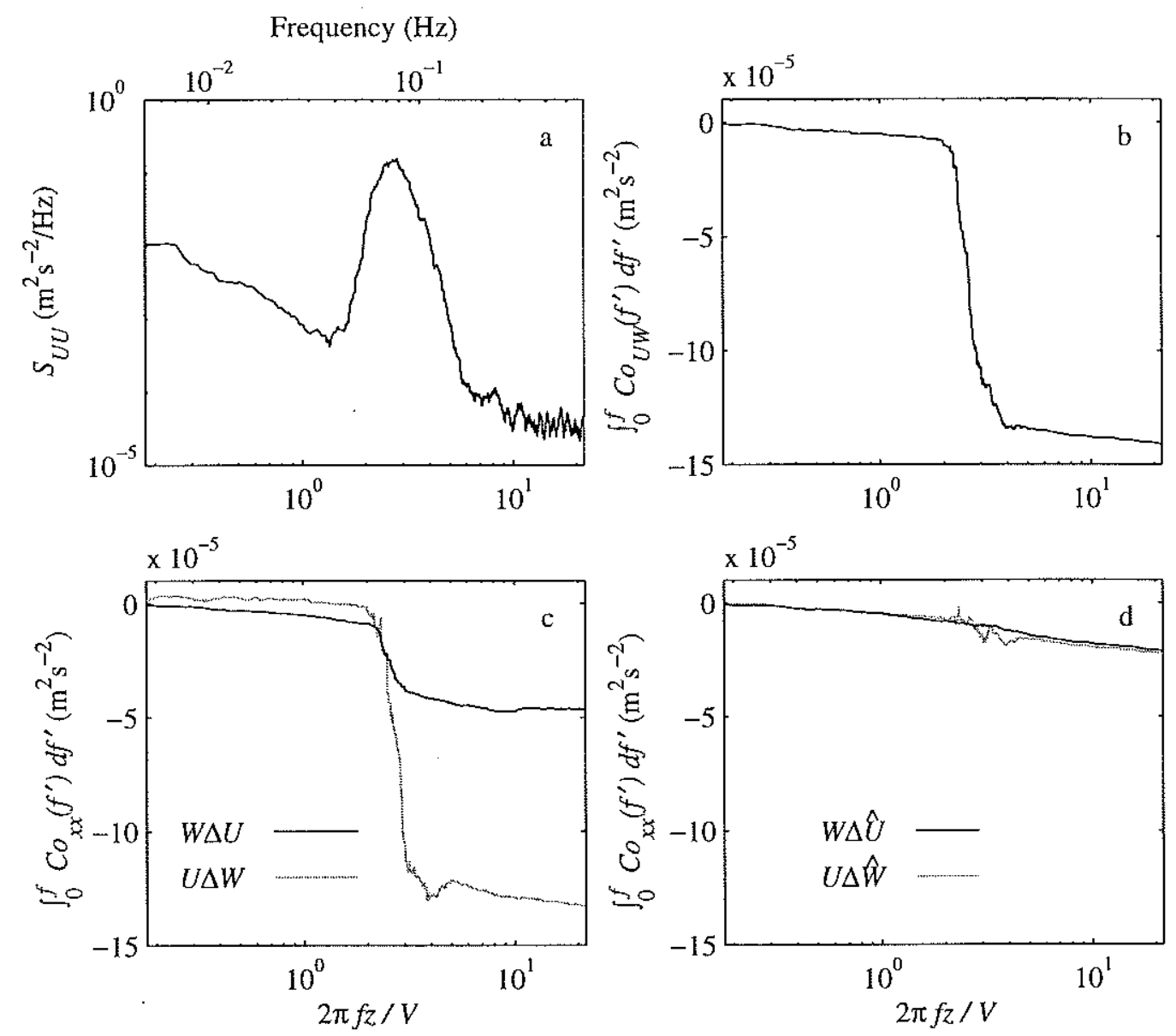

Figure 3.9: Estimates of $\overline{u^{\prime} w^{\prime}}$ under highly energetic wave conditions (surface wave rms velocity $=0.12 \mathrm{~m} / \mathrm{s}$ ) from BASS measurements at $0.70 \mathrm{~m}$ above the bed taken on day 246 as surface wave energy forced by Hurricane Edouard peaked. The spectrum of the longitudinal velocity fluctuations, smoothed with a 21-point boxcar filter, is given in panel (a). In panels (b-d) ogive curves for three sets of stress estimates are presented: 'raw' (b), 'differenced' (c) and 'filtered' (d). 
bottom, captures the passage of an energetic internal wave, with near-bottom velocities greater than $0.1 \mathrm{~m} / \mathrm{s}$ and a duration of approximately $5 \mathrm{~min}$ (Figure 3.10a). The velocity record of the passage of the internal wave is accompanied by a similar event in temperature (see below). The internal wave made a large low-frequency contribution to the 'raw' cospectrum below $2 \pi f z / V=0.02$ and although the 'filtered' estimate is arguably better than the 'raw' estimate, in contrast to the case of surface waves, the proposed techniques are not successful at removing the observed wave bias (Figure $3.10 \mathrm{~b}$ ), which is not too surprising because of the long duration of the internal wave compared to period of surface waves and the length of the burst and because of the intermittent nature of the internal wave. There is also a small surface wave bias visible in the ogive curve of $C o_{\Delta \hat{U} W}$, suggesting that the use of constant filter weights during a burst with non-stationary internal wave properties degrades the ability of the filtering technique to remove surface wave bias. In practice, the range of the internal wave contamination is nearly out of the expected non-dimensional wavenumber range of the energy-containing eddies (Figure 3.7a) and the unwanted internal wave bias can be removed by windowing $\mathrm{Co}_{u w}$.

A comparison of $\overline{u^{\prime} w^{\prime}}$ estimates from the entire six-week long first deployment using $\operatorname{cov}(\Delta \hat{U}, W)$ as a standard demonstrates that the 'raw' shear stress estimates $\operatorname{cov}(U, W)$ are contaminated with surface wave-induced covariance during the energetic surface wave events (Figure 3.11a). As anticipated in Section 3.2.2, the 'differenced' shear stress estimate $\operatorname{cov}(\Delta U, W)$ is mostly free of surface wave contamination during the high-energy wave events, while $\operatorname{cov}(U, \Delta W)$ is arguably worse than $\operatorname{cov}(U, W)$ (Figure $3.11 \mathrm{~b}$ and $\mathrm{c}$ ). The two 'filtered' estimates agree very well (Figure 3.11d). These results indicate that the three estimates $\operatorname{cov}(\Delta U, W), \operatorname{cov}(\Delta \hat{U}, W)$, and $\operatorname{cov}(U, \Delta \hat{W})$ are successful at removing surface 

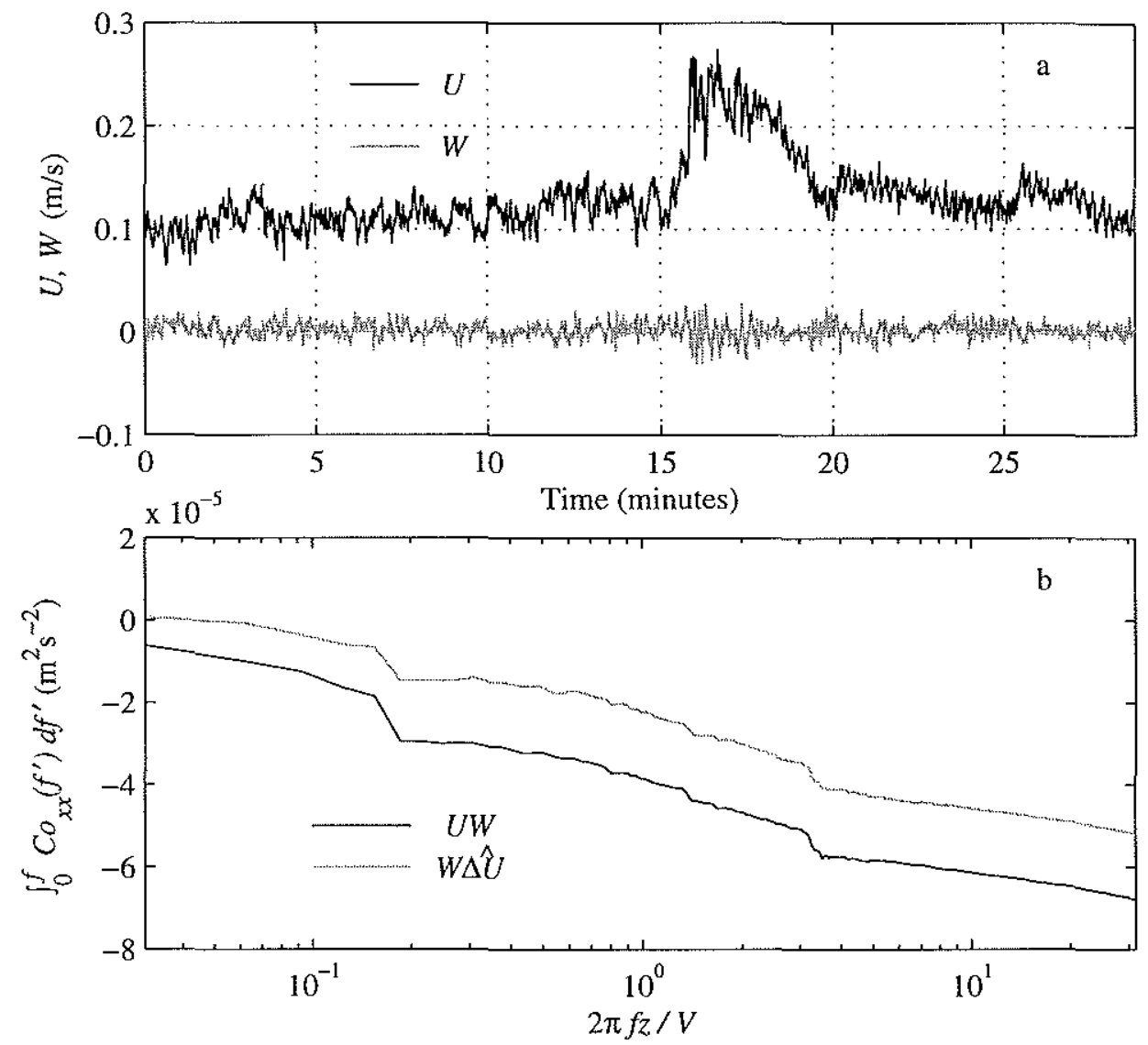

Figure 3.10: Estimates of $\overline{u^{\prime} w^{\prime}}$ under energetic internal wave conditions from a single burst of BASS measurements at $1.10 \mathrm{~m}$ above the bed taken on day 253 during a period of strong stratification. Burst time series of longitudinal and vertical velocity are given in panel (a). In panel (b), ogive curves for $\operatorname{cov}\left(U_{(1)}, W_{(1)}\right)$ and $\operatorname{cov}\left(\Delta \hat{U}, W_{(1)}\right)$ are presented. 
wave bias when it exists and, as shown by comparison with the 'raw' estimate, the successful techniques do not degrade the shear stress estimates when energetic surface waves are absent.

\subsubsection{Heat Flux}

As in the case of shear stress, we consider the cospectra that are integrated to yield the three sets of $\overline{T^{\prime} w^{\prime}}$ estimates described in section (3.3), because wave-induced contributions to heat flux are also observable as deviations from the expected form of $C o_{T w}$ in typical boundary layer flows. Empirical forms for $C o_{T w}$ and the running integral of $C o_{T w}, \int_{0}^{f} C o_{u w}\left(f^{\prime}\right) d f^{\prime}$, (Figure 3.7b) from Kaimal et al. [1972] are similar to those for shear stress, except that the range of heat-flux carrying eddies is shifted to higher $2 \pi f z / V$ in comparison to the range of stress-carrying eddies (Figure 3.7a).

For the most part, the bottom boundary layer is well-mixed during periods of strong surface wave activity. However, near-bottom stratification existed during Hurricane Hortense (Figure 3.3b), which resulted in temperature fluctuations produced by surface waves. As an example of the effect of these wave-induced temperature fluctuations on $C o_{T w}$, we present a temperature spectrum and cospectral ogive curves at $3.3 \mathrm{~m}$ above the bed, position (1) in this case, for a burst in which the rms wave-induced temperature was 0.01 $\operatorname{degC}$ (Figure 3.12). In this particular example, the surface wave peak in the temperature spectrum (Figure 3.12a) is located at the upper end of the expected range of the heat flux carrying eddies (Figure 3.7b). The 'raw' estimate contains an anomalous contribution at the dominant surface wave frequency (Figure $3.12 \mathrm{~b}$ ). The anomaly is visible in both of the 'differenced' estimates (Figure 3.12c). And only in the 'filtered' estimate $\operatorname{cov}\left(T_{(1)}, \Delta \hat{W}\right)$ is 

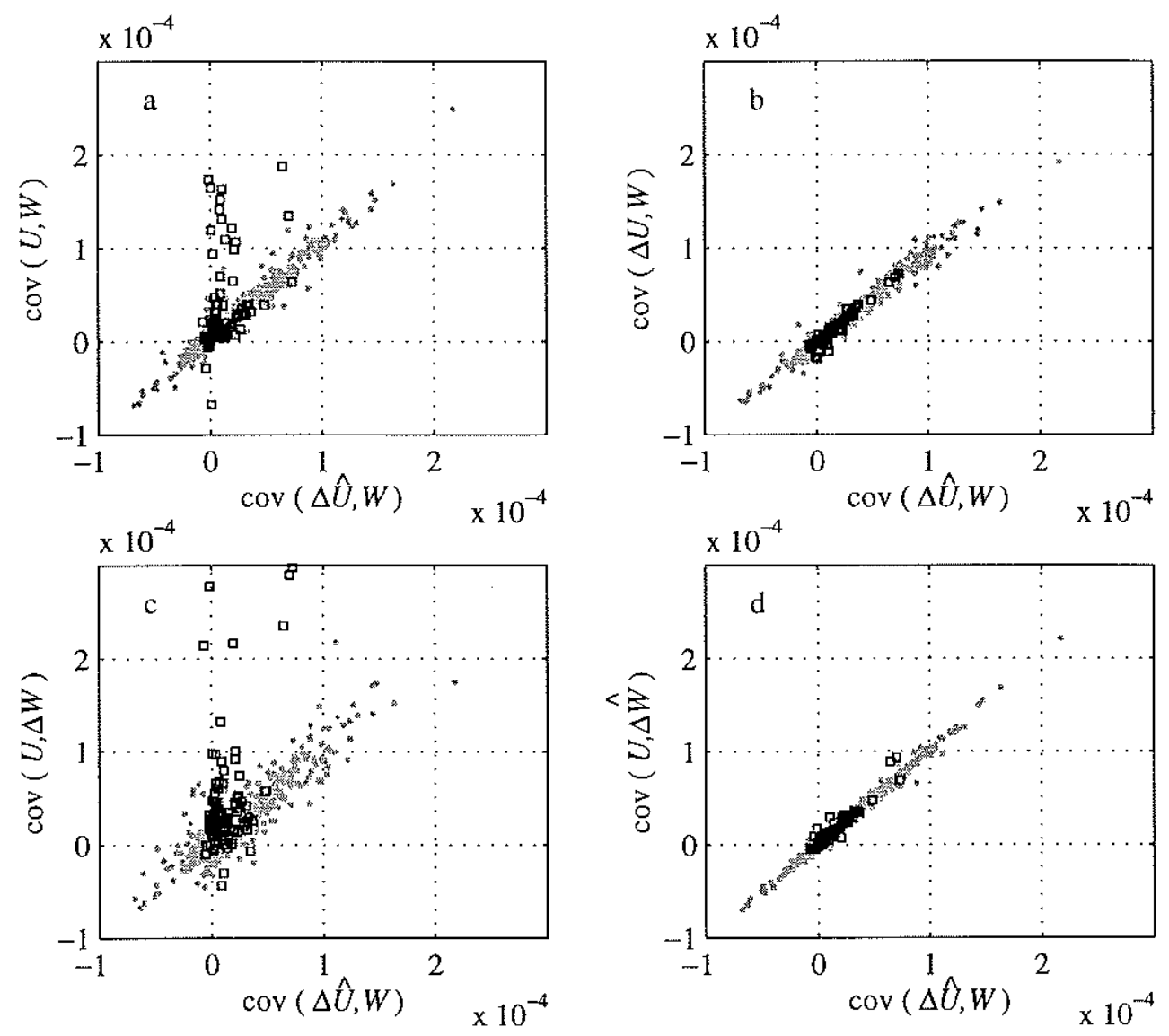

Figure 3.11: Comparison of estimates of $\overline{u^{\prime} w^{\prime}}$ in the along-shelf direction computed from BASS measurements at $0.70 \mathrm{~m}$ above the bed. $\operatorname{cov}(\Delta \hat{U}, W)$ is taken as a standard against which the other estimates are compared: $\operatorname{cov}(U, W)(\mathrm{a}), \operatorname{cov}(\Delta U, W)(\mathrm{b}), \operatorname{cov}(U, \Delta W)(\mathrm{c})$, and $\operatorname{cov}(U, \Delta \hat{W})(\mathrm{d})$. Bursts with surface wave $\mathrm{rms}>0.04 \mathrm{~m} / \mathrm{s}$ are plotted as open squares. The units on all axes are $\mathrm{m}^{2} \mathrm{~s}^{-2}$. 
the observed bias removed (Figure 3.12c). The heat flux carrying eddies in this example are located in a higher range of $2 \pi f z / V$ than expected, which is probably caused by stable stratification limiting the size of eddies (compare with Figure 3.7b). The pronounced effects of surface waves on estimates of heat flux contradict the theoretical scale analysis presented in Section 3.2.1.

The burst containing an internal wave presented in Figure 3.10 is here considered in terms of the effect of the internal wave on estimates of heat flux. The time series of sound speed from $1.1 \mathrm{~m}$ above the bed is nearly constant until disturbed by the passage of the internal wave 16 min into the burst (Figure $3.13 \mathrm{a}$ ). The internal wave made large contributions to 'raw' estimate and the contamination is not eliminated in the 'filtered' estimate (Figure $3.13 \mathrm{~b}$ ) as in the case of shear stress. In fact, both estimates result in a positive heat flux, which is counter-gradient for the stably stratified bottom boundary layer. Note that the sound speed record (Figure 3.13a) is clearly inconsistent with the implicit assumption of stationarity of the statistical properties of waves within individual bursts. Unlike $C o_{u w}, C o_{T w}$ is contaminated well into the expected range of heat flux-carrying eddies, above $2 \pi f z / V=1$ (Figure $3.7 \mathrm{~b}$ ), so that the $C o_{T w}$ cannot be simply windowed to remove internal wave bias without a loss of turbulent flux.

\subsection{Discussion}

The results of Section 5.4 confirm several of the theoretical predictions of Section 3.2. The success of the shear stress estimate $-\rho \operatorname{cov}(\Delta U, W)$ at removing surface wave bias, together with the failure of the estimate $-\rho \operatorname{cov}(U, \Delta W)$, demonstrates the importance of taking 


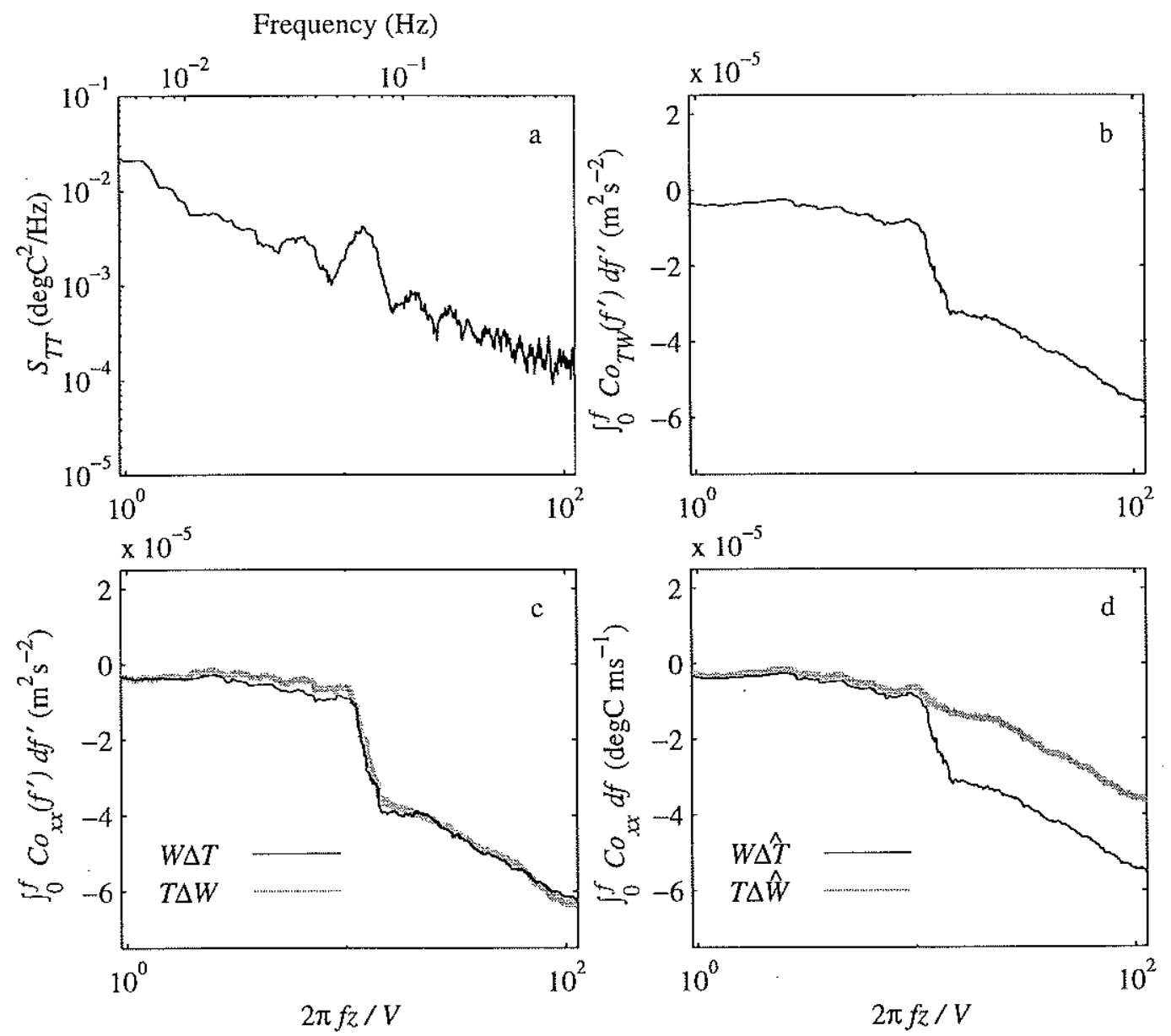

Figure 3.12: Estimates of $\overline{T^{\prime} w^{\prime}}$ under energetic wave conditions and stratification from BASS measurements at $3.3 \mathrm{~m}$ above the bed taken on day 258 during Hurricane Hortense. The spectrum of the temperature fluctuations is given in panel (a). In panels (b-d), ogive curves for three sets of heat flux estimates are presented: 'raw' (b), 'differenced' (c) and 'filtered' (d). 

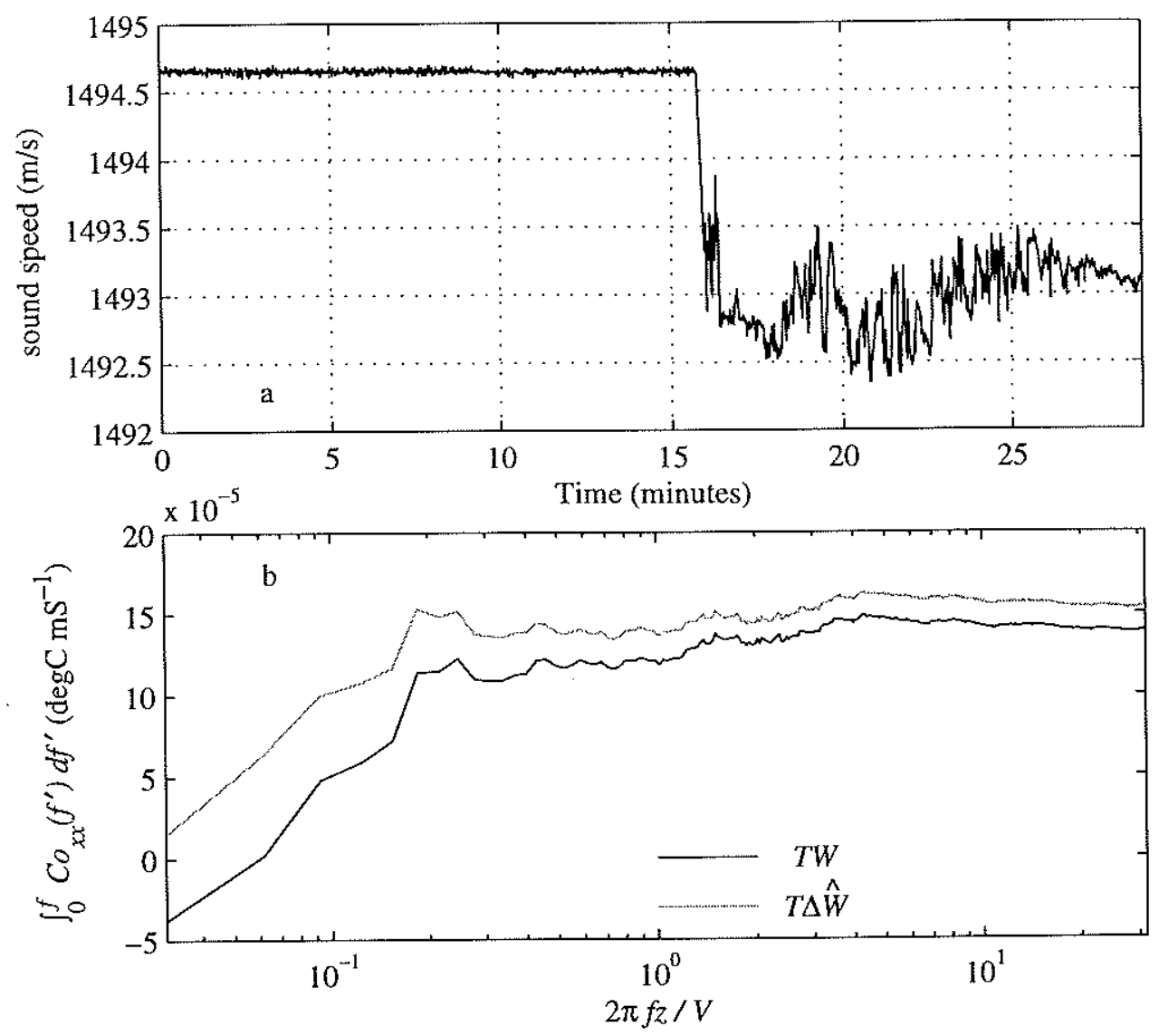

Figure 3.13: Estimates under energetic internal wave conditions from a single burst of BASS measurements at $1.10 \mathrm{~m}$ above the bed taken on day 252 during a period of strong stratification. Burst time series of sound speed is given in panel (a). In panel (b), ogive curves for two heat flux estimates are presented in which the operations are performed on the vertical velocity component. 
advantage of the near-bottom properties of small-amplitude, irrotational surface waves, for which $\tilde{u}$ is approximately independent of $z$ and $\tilde{w}$ increases linearly with $z$ for $k z \ll 1$. The estimate $-\rho \operatorname{cov}(\Delta U, W)$ is predicted to be more effective at reducing wave bias by a factor of $k^{2} z^{2}$, which is approximately $10^{-4}$ for near-bottom measurements at the CMO site with typical $15 \mathrm{~s}$ swell. It is curious, then, why the estimate $-\rho \operatorname{cov}(\Delta U, W)$ fails at the height of energetic surface wave conditions forced by hurricane Edouard (Figure 3.9c). The failure is likely due to either surface waves with higher than usual frequency surface waves reaching the bottom with non-negligible velocities (Figure 3.9a) that encroach on the assumption that $k z<<1$ or local flow disturbances that are produced by the presence of the waves. As noted in Section 5.4, the contamination of $C o_{T w}$ by surface waves (Figure $3.12 \mathrm{~b}$ ) is larger than expected from the estimate (3.4), suggesting that the wall-layer estimate for heat flux used in Section 3.2.1 is not appropriate. The failure of the estimate $\rho c_{p} \operatorname{cov}(\Delta T, W)$ is probably caused by a lack of temperature correlation due to a non-uniform vertical temperature gradient. Because the bottom at the site is smooth and fairly flat and the surface waves are weakly non-linear, we expect that the surface wave contamination was due largely to errors in the levelling of the instruments. The case of internal waves is more complicated and will be discussed later.

In general, the results indicate that wave bias can be reduced by accounting for spatial differences in wave-induced velocity fluctuations with empirically derived transfer functions. In particular, the success of the estimate $-\rho \operatorname{cov}(U, \Delta \hat{W})$ (figure 3.11d) demonstrates that the filtering technique is capable of accounting for the vertical variation of $\hat{w}$ that causes the estimate $-\rho \operatorname{cov}(U, \Delta W)$ to fail (Figure 3.11c). The capability of the filtering technique is further demonstrated by the success of $-\rho \operatorname{cov}(\Delta \hat{U}, W)$ and $-\rho \operatorname{cov}(U, \Delta \hat{W})$ when near- 
bottom surface wave motions were largest (Figure 3.9d) and the estimate $-\rho \operatorname{cov}(\Delta \hat{U}, W)$ failed (figure 3.9c).

In practice, the adaptive filtering technique is insensitive to the exact values chosen for the number of points in the least-squares problem, $M$, or the number of filter weights, $N$, so long as $M \gg N$. A useful rule of thumb is to use a filter length such that $N d t$ equal to a significant fraction of the wave period and to use a number of data points such that $M d t$ is much greater than the time scale of the largest eddies. The filtering technique requires that the turbulence is spatially incoherent at separations $r$, a stronger statement than requiring it to be uncorrelated; however, if turbulence is uncorrelated, it is almost certainly incoherent because eddies quickly lose their identity in turbulent flow. We have found that including the full velocity vector in the prediction of each component of waveinduced velocity yields better stress estimates during demanding wave conditions than if only one velocity component is used for prediction. We have attempted to use a fully recursive filtering technique [e.g., Haykin, 1996] in which the filter weights are continuously updated throughout each burst during periods of internal wave activity, but we found that turbulent covariance was lost because, on short enough time scales, large eddies can be spatially coherent.

None of the proposed techniques were completely successful at removing internal wave bias from estimates of shear stress or heat flux although the internal wave bias for stress estimates can usually be easily removed by high-pass filtering. The 'differenced' stress estimates most likely failed because the near-bottom variation of internal wave-induced velocity was not small, as in the case for the horizontal motions induced by surface waves. The near-bottom structure of internal wave induced- motion is complicated by the fact 
that the height of the internal wave boundary layer is not small relative to the measuring heights, that the slow phase speed of internal waves increases potential for non-linear effects, that rotational effects may be important, and that the periods of the internal waves are of comparable length to the period of measurement. Non-linear effects are especially likely for internal wave-induced temperature fluctuations because the vertical displacements caused by the passage of large internal waves are large enough to advect the density interfaces that tend to develop at the top of the mixed layer (see Figure 3.13a) a considerable distance, making the term $\tilde{w} d \tilde{T} / d z$ large and invalidating the estimate (3.3). This non-linear effect is demonstrated in the extreme example of the time series in Figure $3.13 \mathrm{a}$ and in the spectrum and cospectrum presented in Figures $3.5 \mathrm{~b}$ and $3.13 \mathrm{~b}$ where the effect of internal waves on temperature is apparently transferred to higher frequencies. The 'filtered' estimates fail because non-linearity invalidates the assumptions of the linear filtration techniques and because the intermittency of the internal waves within bursts violates the assumption of stationarity of wave properties. In light of the complicated nature of the observed internal waves it is not clear how much of the internal wave contribution to measured covariances is 'real' or 'apparent'. This is a question we hope to pursue in future work.

From the viewpoint of experimental design, the proposed techniques are valuable because they can be used to obtain surface wave-free flux estimates with a vertical array of sensors, which allows the vertical structure of bottom boundary layer turbulence to be studied. In particular, the technique of differencing a single component of velocity results in a flux estimate at the height of a single sensor as opposed an estimate that is spatially averaged. Without single differencing, it would be difficult to obtain flux estimates at a wide range of heights while satisfying the condition $r>5 z$. We expect that the 'differenced' estimate 
$-\rho \operatorname{cov}(\Delta U, W)$ is adequate for the removal of surface waves from stress estimates under all but the most demanding conditions on the outer shelf. For more energetic conditions, the 'filtered' estimate $-\rho \operatorname{cov}(\Delta \hat{U}, W)$ can be used. To obtain surface wave-free heat flux estimates, we expect that the estimate $\rho c_{p} \operatorname{cov}(T, \Delta \hat{W})$ is neccessary because wave-induced temperature fluctuations are not coherent if $d \bar{T} / d z$ is strongly inhomogeneous. In practice, it is best to remove internal wave energy by high-pass filtering before the filter weights are calculated, because the presence of non-stationary internal waves can degrade the estimation of surface wave-induced motions.

\subsection{Summary and Conclusions}

This paper has presented a theoretical analysis and an oceanic test of techniques to estimate near-bottom turbulence-induced fluxes in the presence of energetic waves motions with current meter arrays. A new adaptive filtering technique has been introduced to minimize the contributions of wave motions to measured covariances. The technique requires two sensors separated in space and requires that the coherency scale of wave motions be much larger than the coherency scale of turbulent motions. The techniques were applied to a sixweek long set of coastal bottom boundary layer observations that include three energetic surface wave events and a number of energetic internal wave events.

Results from the oceanic test indicate the following conclusions. The proposed technique succeeds at removing surface wave contamination from shear stress and heat flux estimates using pairs of sensors separated in the vertical dimension by a distance of approximately five times the height of the lower sensor, even during the close passage of hurricanes, but 
fails at removing internal wave contamination from shear stress and heat flux estimates. The presence of internal waves does not pose a significant problem for estimating turbulent shear stress because contamination caused by internal waves is limited to frequencies lower then the stress carrying eddies. In contrast, the presence of internal waves does pose a problem for estimating turbulent heat flux because the contamination extends in the range of the heat flux carrying eddies. The internal wave case is complicated by the facts that the internal waves are highly intermittent, that the internal wave period is comparable to the period of measurement, that the height of the internal wave boundary layer is of the order of the height of measurement and, specifically for heat flux estimates, that nonlinear effects are large. 


\section{Bibliography}

K. F. Bowden and L. A. Fairbairn. A determination of the frictional forces in a tidal current. Proc. Roy. Soc. A, 214:371-391, 1952.

K. F. Bowden and L. A. Fairbairn. Measurements of turbulent fluctuations and reynold's stresses in a tidal current. Proc. Roy. Soc. A, 237:422-438, 1956.

K. F. Bowden, L. A. Fairbairn., and P. Hughes. The distribution of shearing stresses in a tidal current. Geophys. J. R. Astron. Soc., 2:288-305, 1959.

J. A. Businger, J. C. Wyngaard, Y. Izumi, and E. F. Bradley. Flux-profile relationships in the atmospheric surface layer. J. Atmos. Sci, 28:181-189, 1971.

H. Charnock. Tidal friction from currents near the seabed. Geophys. J. R. Astron. Soc., 2: $215-221,1959$.

J. J. Fredericks, J. H. Trowbridge, A. J. Williams, G. Voulgaris, and W. J. Shaw. Fluid mechanical measurements within the bottom boundary layer during coastal mixing and optics. Technical report, Woods Hole Oceanographic Institution, 1999.

W. D. Grant and O. S. Madsen. The continental-shelf bottom boundary layer. Ann. Rev. Fluid Mech., 18:265-305, 1986. 
W. D. Grant, A. J. Williams, and S. M. Glenn. Bottom stress estimates and their prediction on the northern california continental shelf during code-1: The importance of wavecurrent interaction. J. Phys. Oceanogr., 12:506-527, 1984.

M. O. Green and I. N. McCave. Seabed drag coefficient under tidal currents in the eastern irish sea. J. Geophys. Res., 100:16,057-16,069, 1995.

T. F. Gross and A. R. M. Nowell. Mean flow and turbulence scaling in a tidal boundary layer. Cont. Shelf Res., 2:109-126, 1983.

T. F. Gross and A. R. M. Nowell. Spectral scaling in a tidal boundary layer. J. Phys. Oceanogr., 15:496-508, 1985.

S. Haykin. Adaptive Filter Theory. Prentice Hall, 3rd edition, 1996.

A. D. Heathershaw. The turbulent structure of the bottom boundary layer in a tidal current. Geophys. J. R. Astr. Soc., 58:395-430, 1979.

D. A. Huntley and D. G. Hazen. Seabed stresses in combined wave and steady flow conditions on the nova scotia continental shelf: Field measurements and predictions. J. Phys. Oceanogr., 18:347-362, 1988.

J. C. Kaimal, J. C. Wyngaard, Y. Izumi, and Cote O. R. Spectral characteristics of surfacelayer turbulence. Quart. J. R. Met. Soc., 98:563-589, 1972.

R. G. Lueck and Y. Lu. The logarirhmic layer in a tidal channel. Cont. Shelf Res., 17: $1785-1801,1997$. 
J. L. Lumley and E. A. Terray. Kinematics of turbulence convected by a random wave field. J. Phys. Oceanog., 13:2000-2007, 1983.

K. V. MacKenzie. Nine-term equation for sound speed in the oceans. J. Acoust. Soc. Am., $70: 807-812,1981$.

S. R. McLean and J. D. Smith. Turbulence measurements in the boundary layer over a sand wave field. J. Geophys. Res., 84:7791-7807, 1979.

A. S. Monin and A. M. Yaglom. Statistical Fluid Mecahnics. MIT Press, Cambridge, Mass., 1971.

S. Pond. Some effects of buoy motion on measurements of wind speed and stress. $J$. Geophys. Res., 73:507-512, 1968.

W. J. Shaw, A. J. Williams, and J. H. Trowbridge. Measurement of turbulent sound speed fluctuations with an acoustic current meter. In Oceans96. MTS/IEEE, 1996.

R. L. Soulsby. Similarity scaling of turbulence spectra in marine and atmospheric boundary layers. J. Phys. Oceanog., 7:934-937, 1977.

R. W. Sternberg. Friction factors in tidal channels with differing bed roughness. Mar. Geol., 6:243-260, 1968.

H. Tennekes and J. L. Lumley. A First Course in Turbulence. MIT Press, Cambridge, Mass., 1972.

D. A. Trivett. Diffuse Flow from Hydrothermal Vents. PhD thesis, WHOI/MIT, 1991. 
J. H. Trowbridge. On a technique for measurement of turbulent shear stress in the presence of surface waves. J. Atm. and Ocean. Tech., 15:290-298, 1998.

J. H. Trowbridge, W. R. Geyer, M. M. Bowen, and A. J. Williams. Near-bottom turbulence mesurements in a partially mixed estuary: Turbulent energy balance, velocity structure, and along-channel momentum balance. J. Phys. Oceanogr., in press.

D. C. Twichell, C. E. McClellan, and B. Butman. Morphology and processes associated with the accumulation of the fine-grained sediment deposit on the southern new england shelf. J. Sed. Pet., 51:269-280, 1981.

A. J. Williams, J. S. Tochko, R. L. Koehler, W. D. Grant, Gross T. F., and C. V. R Dunn. Measurement of turbulence in the oceanic bottom boundary layer with an acoustic current meter array. J. Atm. and Ocean. Tech., 4:312-327, 1987. 


\title{
Chapter 4
}

\section{The Budgets of Turbulent Kinetic Energy and Scalar Variance in the Continental Shelf Bottom Boundary Layer}

\author{
William J. Shaw, John H. Trowbridge and Albert J. Williams III \\ (This chapter represents a manucript that is to be submitted to The Journal of Geo- \\ physical Research.)
}

\begin{abstract}
The turbulent kinetic energy (TKE) equation and the turbulent scalar variance (TSV) equation describe the energetics of turbulent velocity fluctuations and the variance of the fluctuations of a scalar contaminant, respectively. Here, a simplified TKE budget, in which production is balanced by dissipation and buoyancy flux, and a simplified TSV balance, in which production equals dissipation, are tested. The calculations are based on sixweek-long records of velocity and sound speed measured between 0.7 and $5.4 \mathrm{~m}$ above bottom on the New England shelf. Estimates of the required fluxes and gradients are direct, while estimates of dissipation rates are indirectly obtained from inertial subrange spectra. Estimates of dissipation rates require consideration of spatial filtering due to a finite sensor volume, temporal aliasing, and a finite instrument noise floor. In the TKE budget, production and dissipation are moderately correlated $\left(r^{2}=0.33\right.$ at $0.9 \mathrm{~m}$ above the bottom and 0.38 at $3.8 \mathrm{~m}$ above the bottom) and roughly consistent in magnitude (regression coefficient $=0.79 \times \div 1.10$ at $0.9 \mathrm{~m}$ above bottom and $0.90 \times \div 1.19$ at $3.8 \mathrm{~m}$ above bottom). Buoyancy flux is not a significant component of the TKE budget at 0.9 $\mathrm{m}$ above the bottom except during a few events, while at $3.8 \mathrm{~m}$ above the bottom the flux Richardson number maintains a roughly constant value of 0.2 . In the TSV budget, production and dissipation are well-correlated $\left(r^{2}=0.60\right.$ at $0.9 \mathrm{~m}$ above the bottom and 0.55 at $3.8 \mathrm{~m}$ above the bottom); however, dissipation is $2.49 \times \div 1.33$ times production
\end{abstract}


at $0.9 \mathrm{~m}$ above the bottom while dissipation is $0.44 \times \div 1.45$ times dissipation at $3.8 \mathrm{~m}$ above the bottom. The imbalance between production and dissipation in the TSV budget is likely due to the divergence of the vertical flux of sound speed variance, a quantity that is difficult to estimate, although the measurements indicate that this term has the correct sign and roughly the magnitude required to close the budget. The absence of a critical flux Richardson number at $0.9 \mathrm{~m}$ above the bottom and the imbalance of the TSV budget at both heights imply inaccuracies in the use of the Osborn and Osborn-Cox models to infer turbulent diffusivities from dissipation rate estimates.

\subsection{Introduction}

The turbulent kinetic energy (TKE) equation and the turbulent scalar variance (TSV) equation describe the energetics of turbulent velocity fluctuations and the variance of fluctuations of a scalar contaminant, respectively. The TKE and TSV budgets are useful for gaining insights about the the dynamics of the turbulent fluctuations; in particular, they are important in the development of turbulence models. From an observational perspective, the equations provide a framework for evaluating and interpreting turbulence measurements.

For a boundary layer flow whose statistical properties are steady and horizontally homogeneous and in which molecular transport in neglected, the TKE equation [e.g., Tennekes and Lumley, 1972] reduces to

$$
-\overline{u^{\prime} w^{\prime}} \frac{\partial u}{\partial z}-\overline{v^{\prime} w^{\prime}} \frac{\partial v}{\partial z}=\frac{\partial}{\partial z}\left(\frac{1}{\rho_{0}} \overline{w^{\prime} p}+\overline{q^{2} w^{\prime}}\right)+\frac{g}{\rho_{0}} \overline{\rho^{\prime} w^{\prime}}+\epsilon
$$

Here, $z$ is the vertical coordinate, $\mathbf{u}=[u, v, 0]$ is the Reynolds-averaged velocity vector, $\mathbf{u}^{\prime}=\left[u^{\prime}, v^{\prime}, w^{\prime}\right]$ is the turbulent velocity vector, $\rho_{0}$ is a reference density, $\rho^{\prime}$ is the turbulent density fluctuation, $g$ is the gravitational acceleration, $q=\frac{1}{2}\left(u^{\prime 2}+v^{\prime 2}+w^{\prime 2}\right)$ is the kinetic energy of the turbulence per unit mass, $\epsilon$ is the molecular dissipation of TKE and an overbar 
represents a Reynolds average. The term on the left side of (4.1) represents the production of TKE by the working of mean flow against the Reynolds stresses. The first term on the right side of (4.1) represents the divergence of a flux due to turbulent pressure-velocity interactions and advection of turbulent kinetic energy by $w^{\prime}$, respectively. The second term is the buoyancy flux and, for stable stratification, represents a loss of turbulent kinetic energy to potential energy. The dissipation term, $\epsilon$, represents a loss of turbulent kinetic energy to the internal energy of the fluid by molecular processes.

With the same assumptions, the TSV equation [e.g., Tennekes and Lumley, 1972] simplifies to

$$
-\overline{c^{\prime} w^{\prime}} \frac{\partial c}{\partial z}=\frac{\partial}{\partial z}\left(\frac{1}{2} \overline{w^{\prime} c^{\prime 2}}\right)+N
$$

Here, $c$ is the Reynolds average of the scalar quantity of interest, $c^{\prime}$ is the corresponding turbulent component and $N$ is the molecular destruction of $\frac{1}{2} c^{2}$. The term on the left side of (4.2) represents the production of turbulent scalar variance from the mean scalar field. The first term on the right side of (4.2) represents the divergence of a flux due to the advection of turbulent scalar variance by $w^{\prime}$. The dissipation term, $N$, represents the destruction of turbulent scalar variance by irreversible molecular processes. The form taken by $N$ is dependent on the quantity considered. Within, we will be analyzing sound speed fluctuations so that $N$ is dependent on the derivatives of temperature and salinity in a complicated way. In the oceanographic microstructure literature, the quantity $\chi=2 N$ is often used. Here, we will follow the convention used in the atmospheric boundary layer literature and work with $N$.

Despite the fundamental importance of the TKE budget in describing the dynamics of 
boundary layer turbulence, there have only been a handful of attempts to try to measure the terms in the TKE budget in the coastal ocean bottom boundary layer. To date, with the exception of a single study, only the simplest TKE balance, in which production is balanced locally by dissipation, has been tested. Gross and Nowell [1985] estimated production and dissipation in a tidal channel and found that the two were in agreement within the estimated error of the measurements. Recently, Trowbridge et al. [in press] and Sanford and Lien [1999] reported estimates of production and dissipation obtained in a tidal estuary and a tidal channel, respectively. Both Trowbridge et al. and Sanford and Lien found that production was balanced by dissipation near the bottom, but Sanford and Lien found that dissipation exceeded production at mid-depth in the tidal channel. Although these studies represent significant achievements, only the simplest TKE balance was tested and, significantly, the buoyancy flux term was not measured. In a unique study of an estuarine bottom boundary layer, Kawanisi and Yokosi [1994] estimated production, dissipation and, by measuring the salt flux with collocated current meters and conductivity probes, buoyancy flux. Kawanisi and Yokosi report large imbalances in the TKE budget that were attributed to vertical turbulent diffusion. All of the above studies were limited to environments with strong tidal flows and, except for the study of Sanford and Lien, to measurements within a few meters of the bottom. In the oceanic bottom boundary layer, the range of heights above bottom in which production is in balance which dissipation and the related question of the significance of the buoyancy flux term are unresolved to date. In particular, the existence of a critical flux Richardson number [e.g., Ellison, 1957], which is fundamental, for example, to the Osborn model [Osborn, 1980] for estimating momentum fluxes from measurements of the dissipation rate of TKE, has not been evaluated. 
A fair amount of attention has been focused on obtaining direct measurements of scalar fluxes in the oceans, including measurements of heat flux in the oceanic thermocline [Moum, 1990, Yamazaki and Osborn, 1990, Fleury and Lueck, 1994], in a tidal channel [Gargett and Moum, 1995] and, with a unique set of measurements, in the boundary layer below drifting pack ice [McPhee, 1992]. To date there have been no definitive tests of the assumption that production and dissipation of scalar quantities are in local balance, although the assumed balance of production and dissipation is fundamental, for example, in the Osborn-Cox model [Osborn and Cox, 1972] for estimating the turbulent diffusivity of scalar quantities. There have been no reported comparisons of any of the terms in scalar variance budgets in the oceanic bottom boundary layer.

In contrast to the oceanic bottom boundary layer, the terms in both the TKE and TSV budgets have been carefully measured in the atmospheric boundary layer [e.g. Wyngaard and Cote, 1971] and the resulting insight has proved valuable in formulating turbulence models. In the wall-layer (surface layer) of the atmospheric boundary layer for stable conditions, it is generally agreed that the TKE budget is balanced locally by production, buoyancy flux, and dissipation and that the TSV budget is balanced locally by production and dissipation, which, for example, has led to simplifications in second-order turbulence closure models [Wyngaard, 1992].

It is clear that our knowledge of bottom boundary layer turbulence dynamics is limited in part by the paucity of measurements of the quantities in the TKE and TSV budgets. The purpose of the present paper is to test local forms of (4.1) and (4.2), in which the flux divergence terms are neglected, with measurements obtained in the bottom boundary layer of the New England shelf. The data set is unique in that records of a scalar quantity, sound 
speed, were obtained in addition to fluid velocity, an approach that is common in atmospheric boundary layer studies [Larsen et al., 1993, Kaimal and Gaynor, 1991] but which has been attempted only once in the ocean [Trivett, 1991]. In addition, the measurements were obtained with a vertical array of sensors up to a height of seven meters above the bottom, a span that encompasses a significant fraction of the boundary layer. Production terms are estimated with direct measurements of the turbulent fluxes and mean gradients. Buoyancy flux is measured semi-directly using an empirical sound speed density relationship. Dissipation terms are estimated indirectly using Kolmogorov's inertial subrange theory.

The remainder of the paper is organized into the following sections: a description of the methods used to estimate the terms in the TKE and TSV budgets (Section 5.3), a presentation and discussion of the results (Sections 5.4 and 5.5), and a summary and conclusions (Section 5.6).

\subsection{Methods}

\subsubsection{Measurements}

The measurements described here were collected as part of the Coastal Mixing and Optics (CMO) experiment during 1996-97. One component of the field experiment consisted of the deployment of a bottom tripod equipped with current meters in order to study the turbulence dynamics of the coastal bottom boundary layer.

The bottom tripod component of the experiment consisted of five deployments spanning August 1996 to August 1997. For the purposes of this paper, we shall limit our attention to the first, six-week long deployment, which began August 17, 1996. The CMO field site 
is located approximately $100 \mathrm{~km}$ south of Martha's Vineyard, MA on a broad section of the New England shelf known as the 'Mud Patch'. The tripod was deployed close the the central mooring of the CMO field site (Figure 4.1) at a depth of approximately $70 \mathrm{~m}$. The 'Mud Patch', as its name suggests, is a relatively low energy environment, with tidal amplitudes small in comparison to those on Georges Bank [Twichell et al., 1981], which is upstream of the area. The featureless bed at the site is composed of fine-grained sediment [Twichell et al., 1981], implying that effect of the bottom on the boundary layer dynamics is straightforward and can be represented with a small, constant roughness length.

The bottom tripod deployed during the CMO experiment [Fredericks et al., 1999] was outfitted with a vertical array of Benthic Acoustic Stress Sensor (BASS) instruments [Williams et al., 1987], a horizontal array of acoustic Doppler velocimeters (ADV)'s, and a pair of conductivity and temperature cells at the elevation of the top and bottom BASS sensors (Figure 4.2). The vertical BASS array, on which the analysis of the present paper is focused, consisted of seven sensors at heights of $0.4,0.7,1.1,2.2,3.3,5.4$, and $7.0 \mathrm{~m}$ above the bed. The BASS sensors were sampled at $1.2 \mathrm{~Hz}$ in 27 minute bursts for a total of 2060 samples per burst. The bursts were taken in two-hour cycles consisting of 3 half-hour periods during which the instruments were sampled followed by one half-hour period during which the instruments were idle.

The BASS sensor determines the three-dimensional velocity vector by measuring the differential travel-time of acoustic pulses traveling in opposite direction along four $15-\mathrm{cm}$ acoustic axes, that will be denoted as paths A, B, C and D (Figure 4.3). Paths A and C and paths $\mathrm{B}$ and $\mathrm{D}$ define two perpendicular planes, the intersection of which lies in the vertical direction. Within each plane, the two paths are perpendicular to each other and oriented 


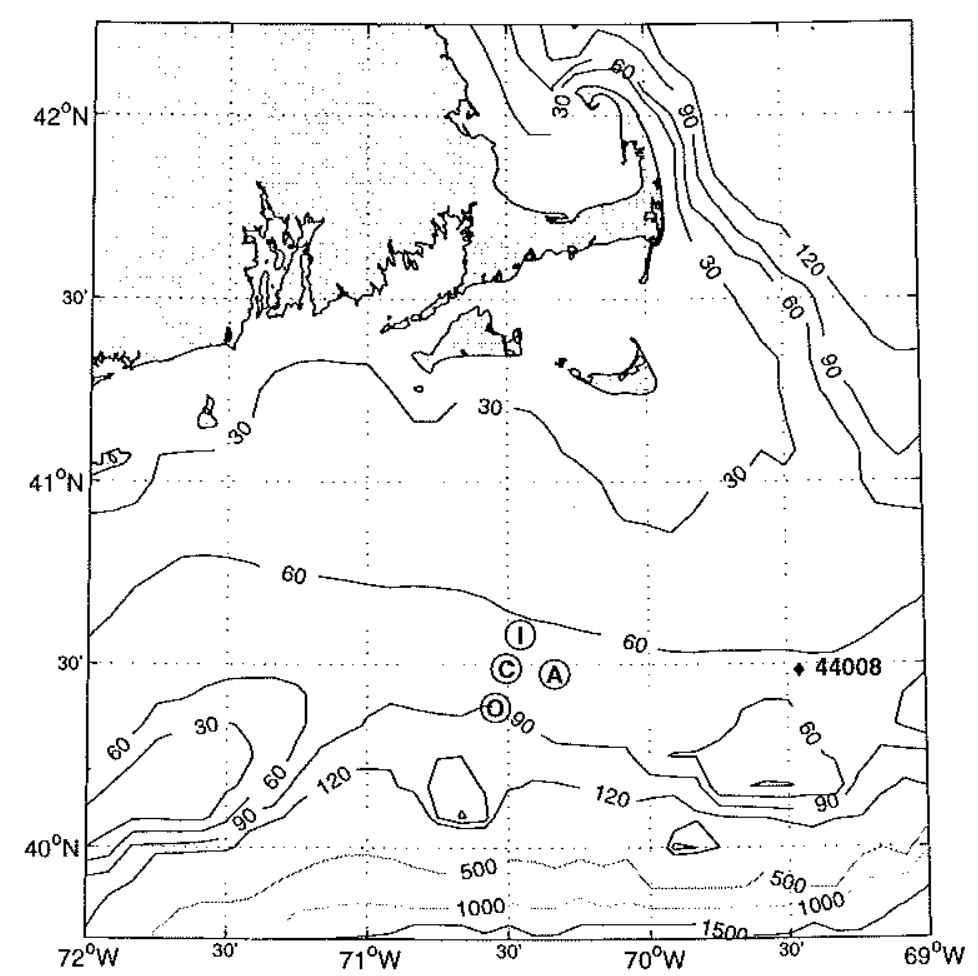

Figure 4.1: Map of the CMO experiment site. Water depth is contoured in meters. The site is approximately $100 \mathrm{~km}$ south of Martha's Vineyard, Massachusetts on a portion of the New England Shelf known as the 'Mud Patch'. Along with the bottom tripod, an array of four moorings were deployed. The tripod was within $200 \mathrm{~m}$ of the central mooring $(\mathrm{C})$, in $70 \mathrm{~m}$ of water. $\mathrm{I}, \mathrm{O}$, and $\mathrm{A}$ represent the locations of in-shore, off-shore, and along-shore moorings, respectively. 


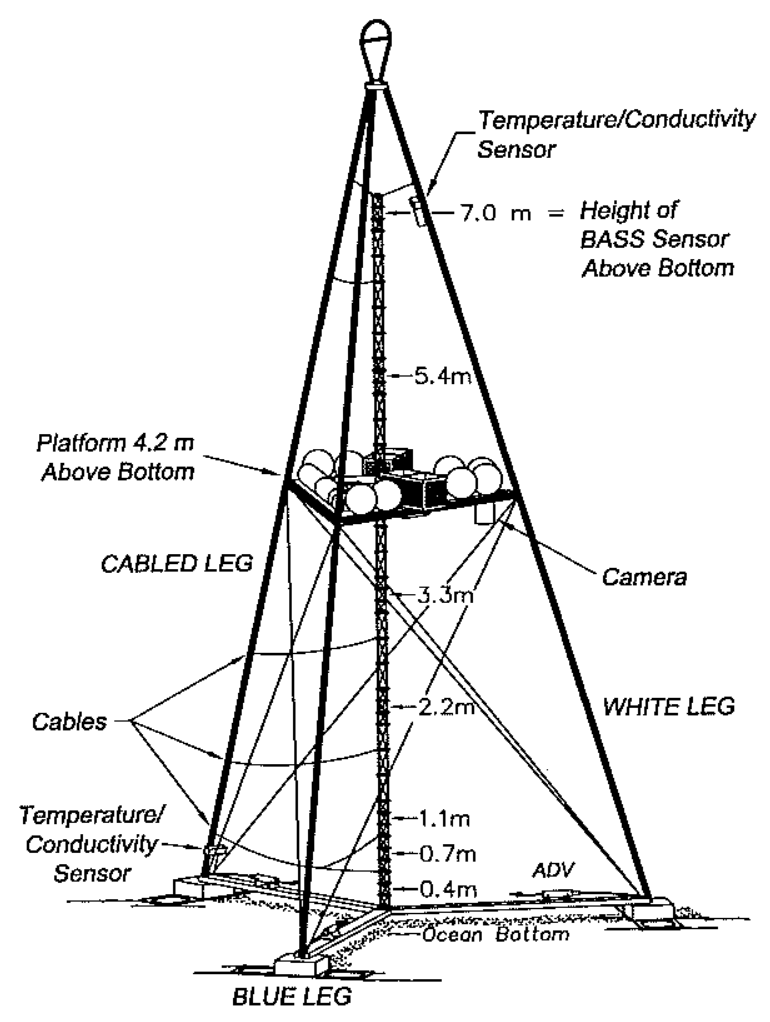

Figure 4.2: Scale drawing of the bottom tripod deployed as a part of the CMO experiment. The tripod was $8 \mathrm{~m}$ tall, containing a vertical array of BASS current meters, a horizontal array of acoustic Doppler velocimeters, and a pair of temperature and conductivity sensors. The heights of the BASS sensors above the bottom are marked. 


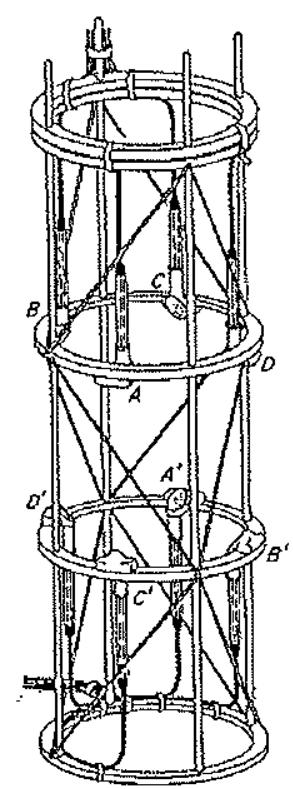

PERSPECTIVE VIEW

Figure 4.3: A scale drawing of a single BASS sensor. Transducers on the upper ring (A, B, $\mathrm{C}$, and $\mathrm{D})$ form acoustic axes with transducers mounted opposite to them on the lower ring $\left(A^{\prime}, B^{\prime}, C^{\prime}\right.$ and $\left.D^{\prime}\right)$. Individual BASS sensors are stacked into a tower to form a vertical array

45 degrees from the vertical direction. The BASS electronics have been recently modified to determine the speed of sound by averaging the absolute travel time of acoustic pulses traveling in opposite directions along an acoustic path [Trivett, 1991, Shaw et al., 1996]. During the CMO deployments, absolute travel-time was measured along path C, yielding a single estimate of sound speed.

Another component of the field study was the deployment of an array of moorings that measured velocity, temperature, and conductivity throughout the water column [Galbraith et al., 1997]. The temperature and conductivity recorded by the bottom-most sensors of the 
central mooring (Figure 4.1), which was located approximately two hundred meters from the SuperBASS tripod [Fredericks et al., 1999], were used to supplement the temperature and conductivity measurements obtained on the tripod.

\subsubsection{Flow Disturbances and Instrument Problems}

In the course of the analysis, we encountered some anomalous measurements that we attributed to instrumentation problems and flow disturbances caused by the structural elements of the tripod. The most troublesome flow disturbance was caused by turbulent wakes, formed in the lee of the tripod legs, that passed through the volumes of the BASS sensors for certain directions of the mean flow. We identified the presence of leg wakes in the sensor volumes by using a statistic based on the variance of the accelerations of the horizontal components of velocity within bursts, $\left(\overline{u_{t}^{2}}+\overline{v_{t}^{2}}\right)$, where a subscript $t$ denotes a time derivative. If $\left(\overline{u_{t}^{2}}+\overline{v_{t}^{2}}\right)$ at each height is normalized by the minimum value of the statistic measured for the burst in the array and plotted as function of flow direction (Figure 4.4), the bursts contaminated by wakes are clearly visible as peaks in the flow directions in which the tripod legs are upstream. The problem was most significant at the upper sensor, where the distance between the sensors and the legs was smallest, and was less severe for the lower sensors. The upper-most sensor, at $7 \mathrm{~m}$ above the bed, was so badly contaminated by wakes that we discarded all measurements from it. For the other sensors, we defined a threshold value for the normalized acceleration, a numerical value of 4 , and burst records that exceeded the threshold were discarded.

In addition to tripod leg flow disturbance, we judged that the Reynolds-averaged velcity measurements from two BASS sensors were faulty due to drifts in zero offset. Offset drift 

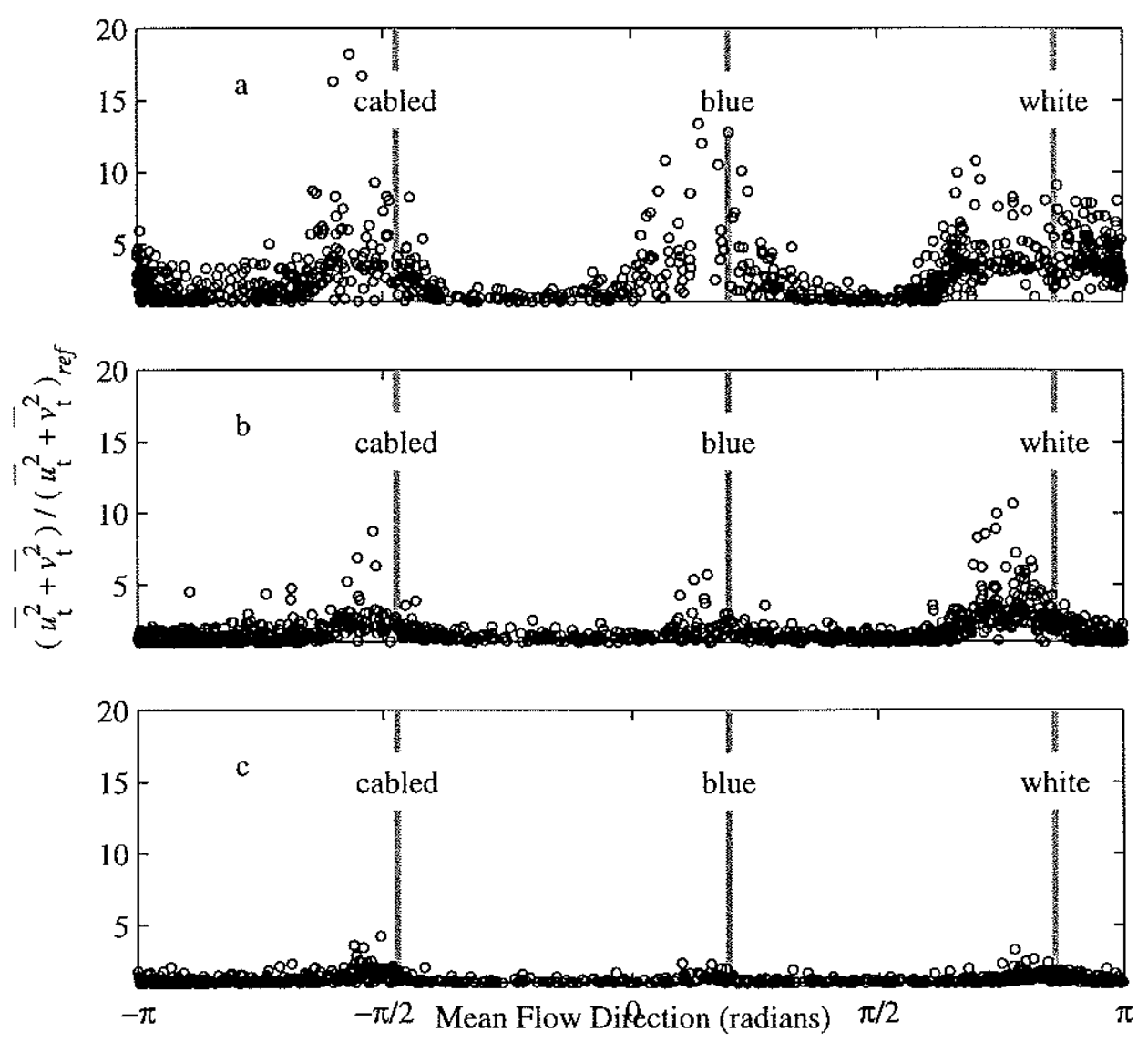

Figure 4.4: Scatter plot of the ratio of the variance of horizontal accelerations to the minimum variance of horizontal acceleration, taken to be a reference, as a function of the angle of the mean flow at the upper three BASS sensors: 7.0 (a), 5.4 (b) and 3.3 (c) $\mathrm{m}$ above bottom. The solid vertical lines indicate the orientation of the tripod legs. 
in the lowest sensor, $0.4 \mathrm{~m}$ above the bottom, was observed in a comparison of redundant estimates of vertical velocity. The sensor at $3.3 \mathrm{~m}$ above the bottom was judged to have drifted based on a comparison of shear estimates at neighboring heights.

Based on these considerations, we discarded all measurements from the top BASS sensor at Reynolds-averaged velocity measurements at heights of 0.4 and $3.3 \mathrm{~m}$ above bottom. We were left with six sensors that recorded turbulent fluctuations reliably at heights of $0.4,0.7$, $1.1,2.2,3.3$, and $5.4 \mathrm{~m}$ and four sensors that recorded burst-averaged velocity and sound speed reliably at heights of $0.7,1.1,2.2$ and $5.0 \mathrm{~m}$.

\subsubsection{Dissipation Rate Estimates}

We obtained dissipation rate estimates by fitting theoretical models to measured inertial subrange spectra. The models include the effects of spatial filtering by finite sensor volume, temporal aliasing, and a noise floor. We used the well-known description of spatial averaging by a line-averaging sensor [e.g., Kaimal et al., 1968] to describe the response of the BASS acoustic paths. The level of the noise floor and the dissipation rate are estimated as part of a two parameter model fitting procedure. We tested a method to correct for the effect of advection of turbulent eddies by surface waves on estimates of $\epsilon$ and $N$ [Lumley and Terray, 1983], but the near-bottom surface wave orbital velocities at the site were generally much smaller than the steady current speeds and we found the corrections to be insignificant.

Spectral processing was performed on each half-hour burst. Burst time series were broken into segments of length $N=248$ points, overlapping by $N / 2$. The segments were windowed with a Hanning window and averaged periodograms, $S_{A A}, S_{B B}, S_{C C}, S_{D D}$ and $S_{c c}$, were computed with approximately 25 degrees of freedom. The measured quantities 
were computed as two-sided power spectra, defined, for example, as

$$
\int_{-\infty}^{\infty} S_{A A}(\omega) d \omega=\overline{u_{A}^{\prime 2}}
$$

where $\omega$ is angular frequency and $u_{A}$ is the component of velocity in the direction of acoustic path A.

We determined the extent of frequencies to which we applied an inertial subrange model by requiring that $\omega>4 \omega_{0}$ and $\omega>2 \pi U / z$, where $z$ is the elevation above the bed of the sensor, $U$ is the magnitude of the mean current and $\omega_{0}$ the dominant surface wave frequency, estimated as the ratio of the first to zeroth moments of the spectra below the observed wave peak. The first condition is required so that the estimates are not affected by surface wave variance and the second condition is the low-frequency limit of the inertial subrange observed in the atmospheric boundary layer [e.g., Kaimal et al., 1972]. On the high-frequency side, we were limited by the Nyquist frequency, $\omega_{N}$, or by requiring that $\omega L / U<5$, where $L$ is the length of the acoustic path, so that the spatial filtering correction was not greater than approximately an order of magnitude.

The frequency range meeting the above restrictions was assumed to correspond to the inertial subrange, and models of the four velocity spectra (along each path) and the single sound speed spectrum were computed at each BASS sensor for each burst. Batchelor [1951] has presented expressions for the one-dimensional wavenumber spectra of isotropic turbulence in the longitudinal and transverse directions. Using the general form for the spectrum tensor of an isotropic velocity field [Batchelor, 1951] and the form of the energy spectrum in the inertial subrange [Tennekes and Lumley, 1972], it can be shown that the 
wavenumber spectrum of the component of inertial subrange velocity fluctuations oriented at an arbitrary angle to the direction of the mean flow is

$$
S_{\eta \eta}\left(k_{1}\right)=\frac{9}{55}\left(\frac{4-\cos ^{2} \theta}{3}\right) \alpha \epsilon^{2 / 3} k_{1}^{-5 / 3}
$$

where $S_{\eta \eta}\left(k_{1}\right)$ is the spectrum as a function of longitudinal wavenumber, $k_{1}$, of the $\eta$ component of velocity fluctuations which are oriented at an angle $\theta$ from the direction of the mean flow, $\alpha$ is the empirical Kolmogorov constant for velocity, and the convention of no summation over Greek indices in employed. The corresponding model of the spectra of scalar contaminants [e.g., Tennekes and Lumley, 1972] is

$$
S_{c}\left(k_{1}\right)=\frac{3}{5} \beta N \epsilon^{-1 / 3} k_{1}^{-5 / 3}
$$

where $S_{c}\left(k_{1}\right)$ is the longitudinal wavenumber spectrum of a scalar contaminant and $\beta$ is the empirical Kolmogorov constant for scalars.

Measured frequency spectra were related to wavenumber spectra via the frozen turbulence hypothesis, $k_{1}=\omega / U$. In addition, the measured frequency spectra are subject to the effects of a non-ideal sensor mentioned above. Accounting for these effects, the system of equations to be solved for $\epsilon$ and the velocity spectral noise floor are

$$
S_{\eta \eta}(\omega)=A_{\eta}\left[\omega^{-5 / 3} T_{\eta \eta}\left(\frac{\omega L}{U}, \theta\right)+\left(2 \omega_{N}-\omega\right)^{-5 / 3} T_{\eta \eta}\left(\frac{\left(2 \omega_{N}-\omega\right) L}{U}, \theta\right)\right]+B_{\eta}
$$

Here, $A_{\eta}=\frac{9}{55}\left(\frac{4-\cos ^{2} \theta}{3}\right) \alpha \epsilon^{2 / 3} U^{2 / 3}, B_{\eta}$ is the noise floor of the $S_{\eta \eta}$ spectrum and $T_{\eta \eta}$ is the vector quantity line-averaging transfer function [Kaimal et al., 1968], which is dependent 
on the non-dimensional quantity $\omega L / U$ and $\theta$. The second term in the square brackets represents a single reflection about $\omega_{N}$ of the inertial subrange spectra and it is included as an approximation of temporal aliasing. The corresponding system of equations to be solved for $N$ and the sound speed spectral noise floor are

$$
S_{c}(\omega)=A_{c}\left[\omega^{-5 / 3} T_{c}\left(\frac{\omega L}{U}, \theta\right)+\left(2 \omega_{N}-\omega\right)^{-5 / 3} T_{c}\left(\frac{\left(2 \omega_{N}-\omega\right) L}{U}, \theta\right)\right]+B_{c}
$$

where $A_{c}=\frac{3}{5} \beta N \epsilon^{-1 / 3} U^{2 / 3}, B_{c}$ is the noise floor of the $S_{c}$ spectrum and $T_{c}$ is the scalar quantity line-averaging transfer function [Silverman, 1968], which is also dependent on $\omega L / V$ and $\theta$. Given the geometry of the BASS sensor with horizontal near-bottom burst-averaged velocities, $\theta$ varies between $\frac{\pi}{4}$ and $\frac{\pi}{2}$.

The unknown constants are determined from the least-squares solutions of the above equations, from which the dissipation rates are determined. For the estimation of $N$, we took the required value of $\epsilon$ as the average of the inertial subrange dissipation estimates from the four acoustic paths. In a review of atmospheric measurements in which dissipation estimates were directly measured or inferred from variance budgets, Hogstrom [1996] reports that the best estimates of the Kolmogorov constants are, in our notation, $\frac{18}{55} \alpha=0.51$ and $\frac{6}{5} \beta=0.8$, corresponding to $\alpha=1.56$ and $\beta=0.67$ which are the values used here.

In order to illustrate the inertial subrange dissipation estimates, we present example velocity spectra from acoustic paths $\mathrm{A}$ and $\mathrm{C}$ and an example sound speed spectrum from a single burst at $1.1 \mathrm{~m}$ above the bottom during a period of strong mixing of momentum and sound speed (Figure 4.5a). $S_{A A}$ and $S_{C C}$ contain an energetic surface wave peak centered about a frequency of $0.07 \mathrm{~Hz}$, corresponding to $15 \mathrm{sec}$ waves. Within the inertial subrange, 
$S_{A A}$ and $S_{C C}$ are predicted to be equal because they are oriented at the same angle to the mean flow. For $\omega z / U>2 \pi$, the two spectra are in close agreement, indicating that the turbulence is isotropic. The slope of the velocity spectra are somewhat greater than $-5 / 3$, which is likely the result of the effects of a finite noise floor and temporal aliasing. A fit to $S_{A A}$ based on (4.6) indicates that the shape of the measured spectrum can be modeled if sensor noise, spatial filtering and aliasing are considered. An example sound speed spectrum at the same time and location (Figure $4.5 \mathrm{~b}$ ) has a well-developed inertial subrange. As in the case of the velocity spectra, the slope of $S_{c}$ is greater than $-5 / 3$, but the spectrum is well described by (4.7). In contrast to this example, the spectrum of sound speed is flat at high frequencies when the boundary layer is well-mixed because $\mathrm{N}$ is close to zero (not shown).

\subsubsection{Flux Estimates}

Estimates of turbulent momentum and sound speed flux were obtained directly from records of velocity and sound speed from each burst by eddy correlation and the use of a linear filtration and differencing technique to remove contamination by surface waves [Shaw and Trowbridge, submitted] which requires the use of pairs of spatially separated sensors. Of the four BASS sensors that obtained reliable turbulence measurements, sensors 2 and 5 were paired together and sensors 3 and 6 were paired together. Although the surface waves had a negligible effect on the dissipation rate estimates, they did cause significant contamination of Reynolds stress and sound speed flux estimates, which was successfully removed by the application of the filtering and differencing technique [Shaw and Trowbridge, submitted].

As noted by Shaw and Trowbridge [submitted], the contamination of heat flux estimates 

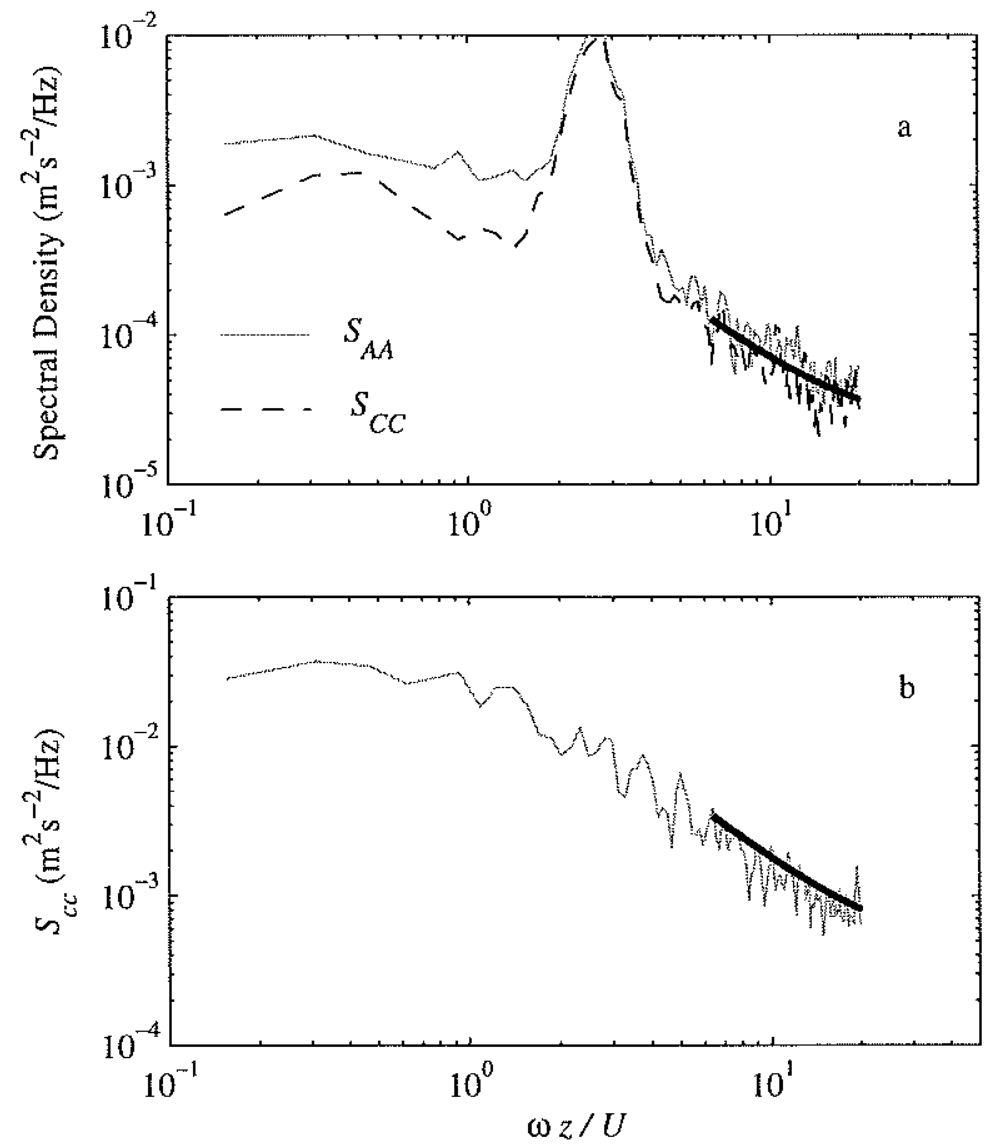

Figure 4.5: Example spectra of velocity fluctuations (a) and sound speed (b) from a single burst at $1.1 \mathrm{~m}$ above the bottom during a period of strong mixing of momentum and sound speed. The thick solid lines represent model fits to the measured spectra. In (a), a single model is fit to $S_{A A}$. These example spectra are from a single half hour burst obtained on day 259, a period of large dissipation rates of TKE and TSV. 
by internal wave-induced velocities is a lingering problem. The symptom of internal wave contamination is noisy estimates of $\overline{c^{J} w^{\prime}}$ that are often counter-gradient. A simple procedure that produces reasonable results is to use the estimate $\overline{c^{\prime} w^{\prime}}=\overline{c_{t}^{\prime} w_{t}^{\prime}} /<\omega^{2}>$, where a subscript $t$ denotes time differentiation and $\left\langle\omega^{2}\right\rangle$, defined by

$$
<\omega^{2}>=\left(\frac{2 \pi U}{z}\right)^{2} \frac{\int_{-\infty}^{\infty} k_{1}^{2} S_{c w}(\omega) d \omega}{\int_{-\infty}^{\infty} S_{c w}(\omega) d \omega}
$$

is the second moment about $k_{1}=0$ of $S_{c w}$, the cospectrum between $c$ and $w$. This estimate of $\overline{c^{\prime} w^{\prime}}$ effectively filters the effect of internal waves which tend to influence the low-frequency part of the spectrum. Based on wall layer scaling, the ratio of integrals in (4.8) is expected to be a universal constant, i.e. the shape of the spectrum is invariant when scaled with $k_{1}$, and $\left\langle\omega^{2}\right\rangle$ is therefore expected to be proportional to $(U / z)^{2}$. In practice, scaling $\overline{c_{t}^{\prime} w_{t}^{\prime}}$ with $(U / z)^{-2}$ did not help to collapse the data when compared to $\overline{c^{\prime} w^{\prime}}$ during periods without internal waves. Instead, $\left\langle\omega^{2}\right\rangle$ was estimated as a function of $z$ only by directly regressing $\overline{c_{t}^{\prime} w_{t}^{\prime}}$ against $\overline{c^{\prime} w^{\prime}}$ for each sensor during periods that were free of internal wave contamination. We found that $\left\langle\omega^{2}\right\rangle$ was equal to between 1 and $1.5(\mathrm{rad} / \mathrm{s})^{2}$, depending on the height of the measurements. The failure of wall scaling is likely due to the importance of turbulence length scales in addition to the height above bottom.

The presence of internal waves is detectable in a time series of the burst standard deviation of horizontal velocity as intermittent periods of large variance (Figure 4.6a). The corresponding time series of $\overline{c^{\prime} w^{\prime}}$ and the estimate of the sound speed flux obtained with $\overline{c_{t}^{\prime} w_{t}^{\prime}}$ and the empirical correction $\left\langle\omega^{2}\right\rangle$ (Figure 4.6b) indicate that when internal waves are absent (e.g., days 245-250) the two estimates are nearly equal and that when internal 
waves are present (e.g., days 253-257) $\overline{c^{\prime} w^{\prime}}$ is severely contaminated, often taking the sign opposite to that expected for stable stratification, while $\overline{c_{t}^{\prime} w_{t}^{\prime}} /<\omega^{2}>$ appears to be free of internal wave contamination (Figure 4.6). Because the differentiation technique succeeds at removing internal wave contamination, $\overline{c_{t}^{\prime} w_{t}^{\prime}} /<\omega^{2}>$ was used as the sound speed flux estimate.

To estimate the buoyancy flux term, we converted the measured sound speed fluctuations into density fluctuations using an empirical linear relationship. We estimated the constant $\frac{\partial \rho}{\partial c}$ as $\frac{\Delta \rho / \Delta z}{\Delta c / \Delta z}$, where the $\Delta$ sign notation means a vertical difference, for each half hour burst. Density differences were estimated using temperature and conductivity measurements obtained on the CMO central mooring (Figure 4.1) which were judged to be more reliable than those obtained from the temperature and conductivity sensors mounted on the bottom tripod. In particular, the bottom conductivity sensor on the tripod was believed to have been fouled by an accumulation of sediment.

From the four BASS sensors that reliably recorded mean and fluctuating quantities, we calculated two sets of production, dissipation and buoyancy flux estimates at $0.9 \mathrm{~m}$ above the bed, from the records obtained by sensors two and three, and at $3.8 \mathrm{~m}$, from the records obtained by sensors four and six. The estimates were made using a vertical average of flux and dissipation rate estimates at the two pairs of sensors and a 2-point difference to approximate the vertical gradients. In a modest attempt to reduce random variability, the flux, gradient, and dissipation rate estimates from the two-hourly cycles of three consecutive bursts were averaged in time. The production terms were computed after the temporal averaging. 

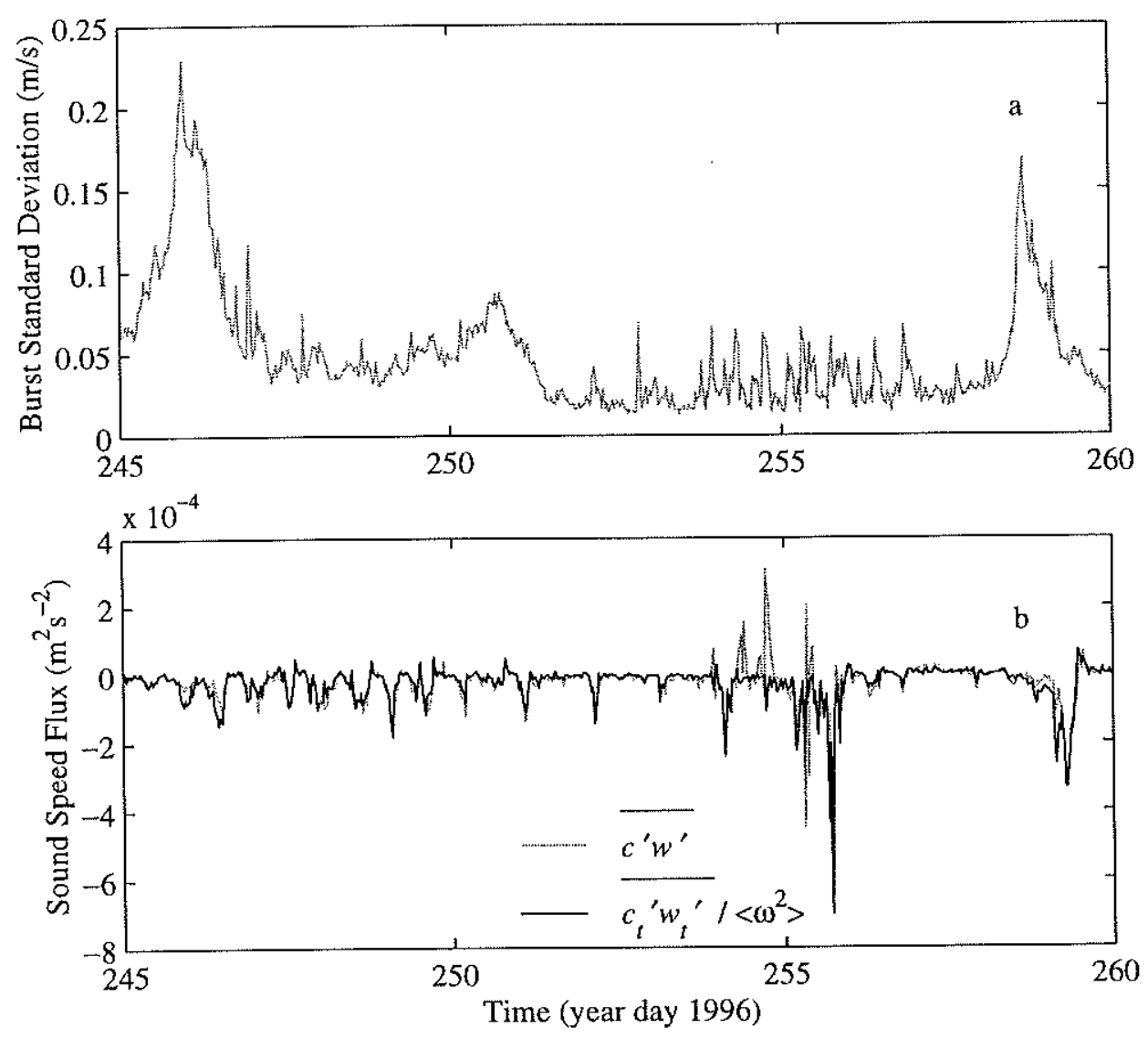

Figure 4.6: Time series of the standard deviation of horizontal velocity fluctuations and $\sigma_{1}$ (a) and the heat flux estimates $\overline{c_{t}^{\prime} w_{t}^{\prime}}$ and $\overline{c^{\prime} w^{\prime}}$ (b) at an elevation of $1.1 \mathrm{~m}$ above the bed. During periods of internal wave activity, visible as high frequency events in the time series of the standard deviation of horizontal velocity, the estimate $\overline{c^{\prime} w^{\prime}}$ suffers from internal wave contamination apparent as counter-gradient (i.e., positive) values of sound speed flux, while the estimate $\left.\overline{c^{\prime} w^{\prime}} /<\omega^{2}\right\rangle$ is apparently free of internal wave contamination. 


\subsection{Results}

\subsubsection{Conditions at the CMO Site}

Before proceeding to a test of the simplified variance budgets, it is worthwhile to briefly describe the conditions at the CMO field site during the first deployment. As stated in Shaw and Trowbridge [submitted], time series of near-bottom mean currents at the CMO site (Figure 5.3a) are usually dominated by a rotary semi-diurnal tide with an amplitude of approximately $0.1 \mathrm{~m} / \mathrm{s}$. Sub-tidal events consist of predominantly westward flows with velocities up to $0.2 \mathrm{~m} / \mathrm{s}$. Time series of mean sound speed measured by the top and bottom BASS sensors (Figure 5.3b) indicate that the bottom boundary layer at the CMO site is usually well-mixed in sound speed. Occasionally, however, near-bottom stratification is strong, resulting in sound speed differences between 0.38 and $5.50 \mathrm{~m}$ above the bed of up to $10 \mathrm{~m} / \mathrm{s}$, which roughly corresponds to $5 \mathrm{degC}$. In particular, there are three events centered on days 255,259 , and 268 during which strong stratification is apparently advected through the measurement area.

\subsubsection{TKE budget}

Time series of the near-bottom estimates of production and dissipation of TKE (Figure 4.8a) contain tidal variability modulated by the strength of sub-tidal current, with peak values of approximately $4-5 \times 10^{-6} \mathrm{~W} / \mathrm{kg}$ during the strong westward sub-tidal flow of days $245-250$. A scatter plot of production vs. dissipation (Figure 4.8b) indicates that there is a noise floor in the dissipation estimates of approximately $10^{-7} \mathrm{~W} / \mathrm{kg}$, but that above the noise floor, the data points cluster about the one-to-one line. Quantitative comparisons are made in terms 

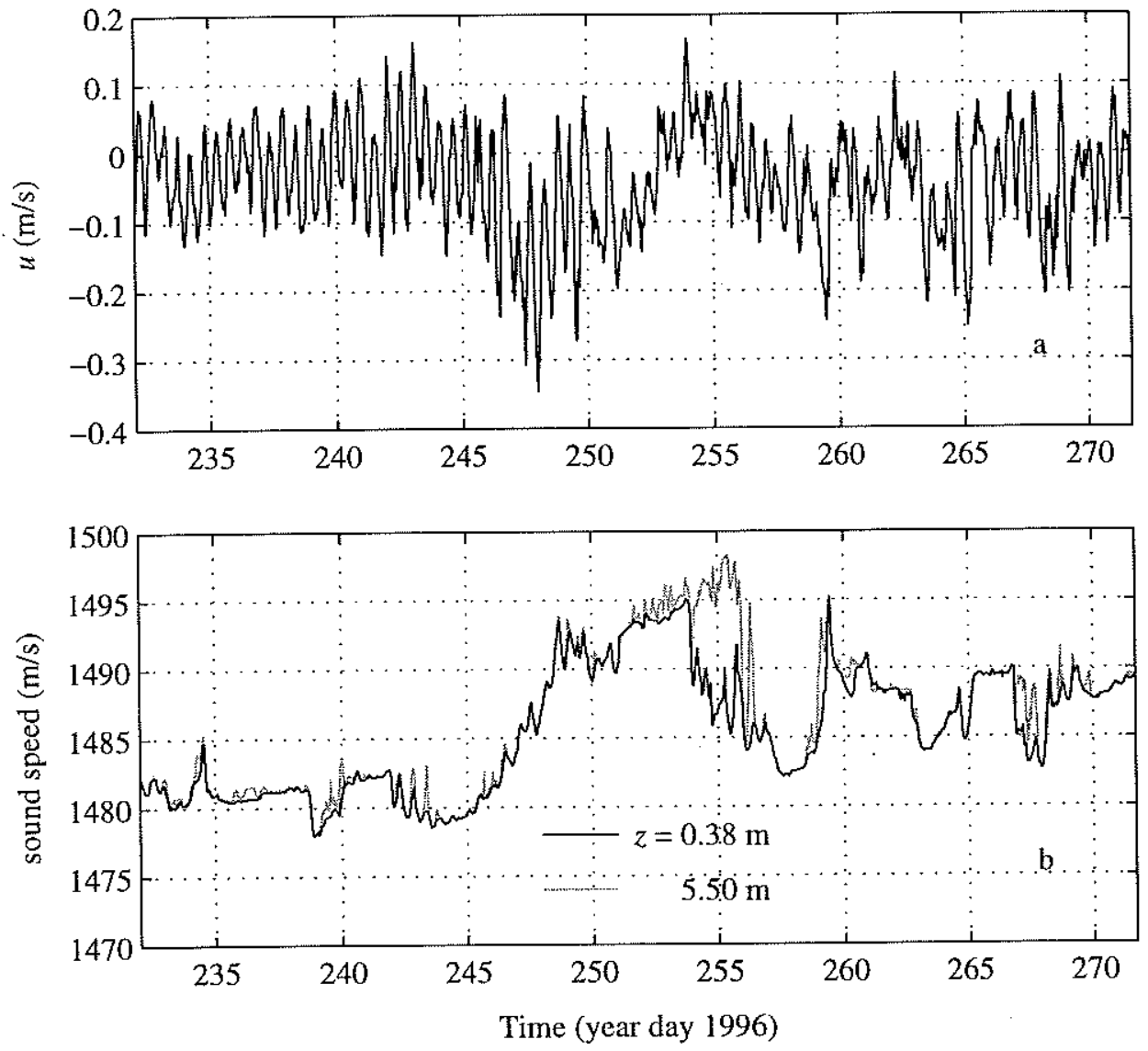

Figure 4.7: Time series of burst-averaged along-shelf current (a) and burst-averaged sound speed (b) measured by the BASS array during the first deployment of the CMO experiment. Statistics are from 27 minute bursts. The coordinate system is oriented so that eastward flow is positive. 
of log-transformed variables and, to avoid biasing statistics with data points that lie in the dissipation noise floor, comparisons are limited to values of production and dissipation greater than $10^{-7} \mathrm{~W} / \mathrm{kg}$. Production and dissipation are moderately correlated $\left(r^{2}=0.33\right.$, for 268 data points) and are roughly consistent in magnitude (regression coefficient $=0.79 \times$ $\div 1.10$ ). The buoyancy flux term is small compared to production and dissipation and including it in the budget does not quantitatively affect the correlation coefficient.

Time series of the upper estimates of production and dissipation (Figure 4.9a) contain a similar tidal variability except that the dissipation estimates are often significantly larger than the production estimates which is also evident in a scatter plot (Figure $4.9 \mathrm{~b}$ ). The anomalously large dissipation rate estimates are most likely the result of enhanced spectral levels at the upper sensors caused by tripod leg wakes. The dissipation estimates are obtained in a higher range of frequency than the flux estimates and are more likely to be affected by the small scale eddies in the leg wakes. The correlation between production and dissipation is similar to the near bottom estimates $\left(r^{2}=0.38\right.$, for 92 data points) and the magnitudes of the estimates are closer to agreement (regression coefficient $=0.90 \times \div 1.19$ ) than at the near-bottom position, although this is probably fortuitous, owing to the flow disturbance biases that increase the dissipation estimates. In comparison to the estimates at $0.9 \mathrm{~m}$ above the bottom, the buoyancy flux term is larger and relatively more significant in comparison to production. The vertical structure of the production estimates is qualitatively consistent with wall layer scaling, with larger values closer to the bed, as expected.

We can use the estimates of TKE production and buoyancy flux to test for the existence of a critical flux Richardson number. After interpolating through gaps in the time series caused by flow disturbances and low-pass filtering using the routine pl64 [Limeburner, 1985], 

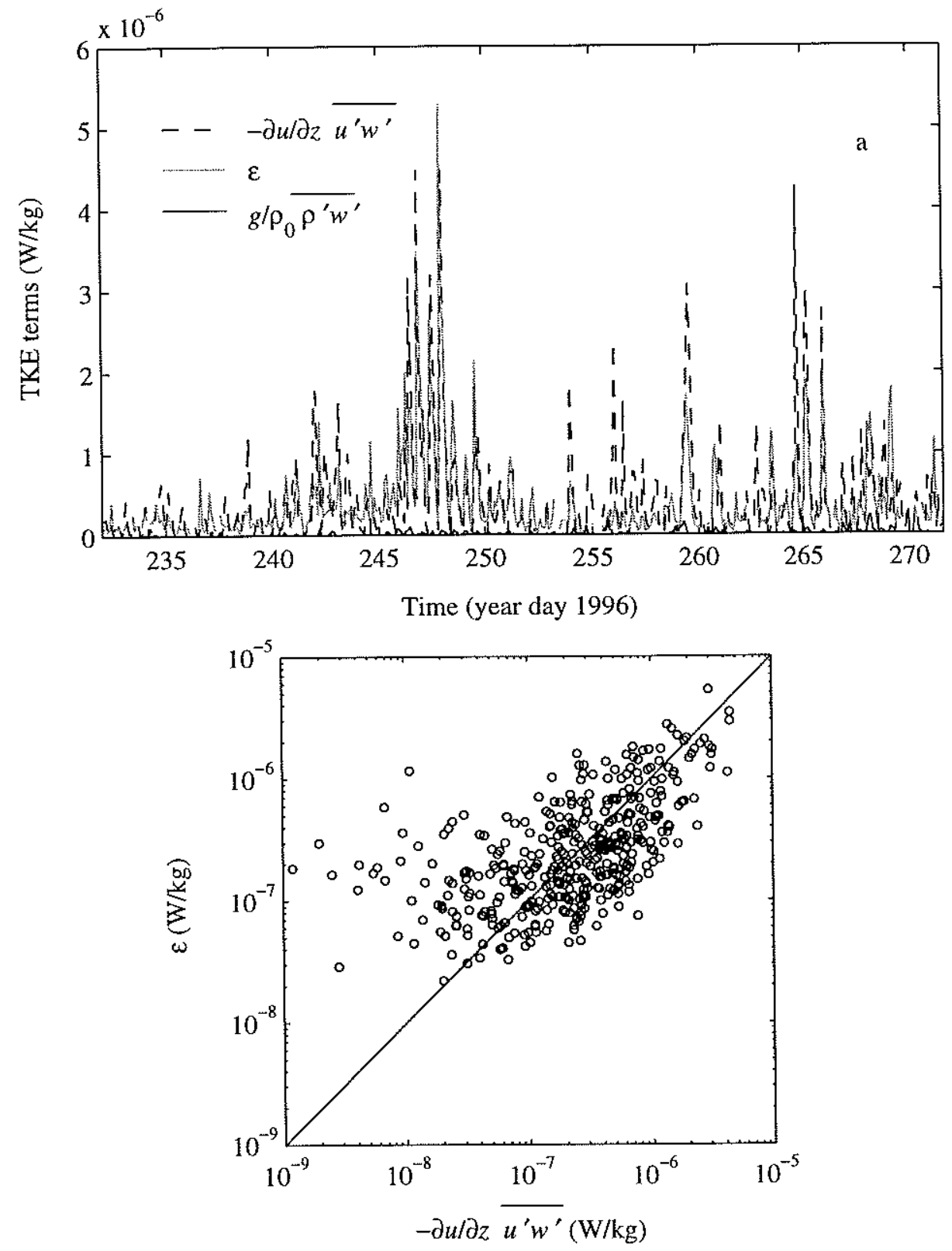

Figure 4.8: Time series (a) and scatter plot (b) of the production and dissipation terms in the TKE budget estimated at a height of $0.9 \mathrm{~m}$ above the bed. 

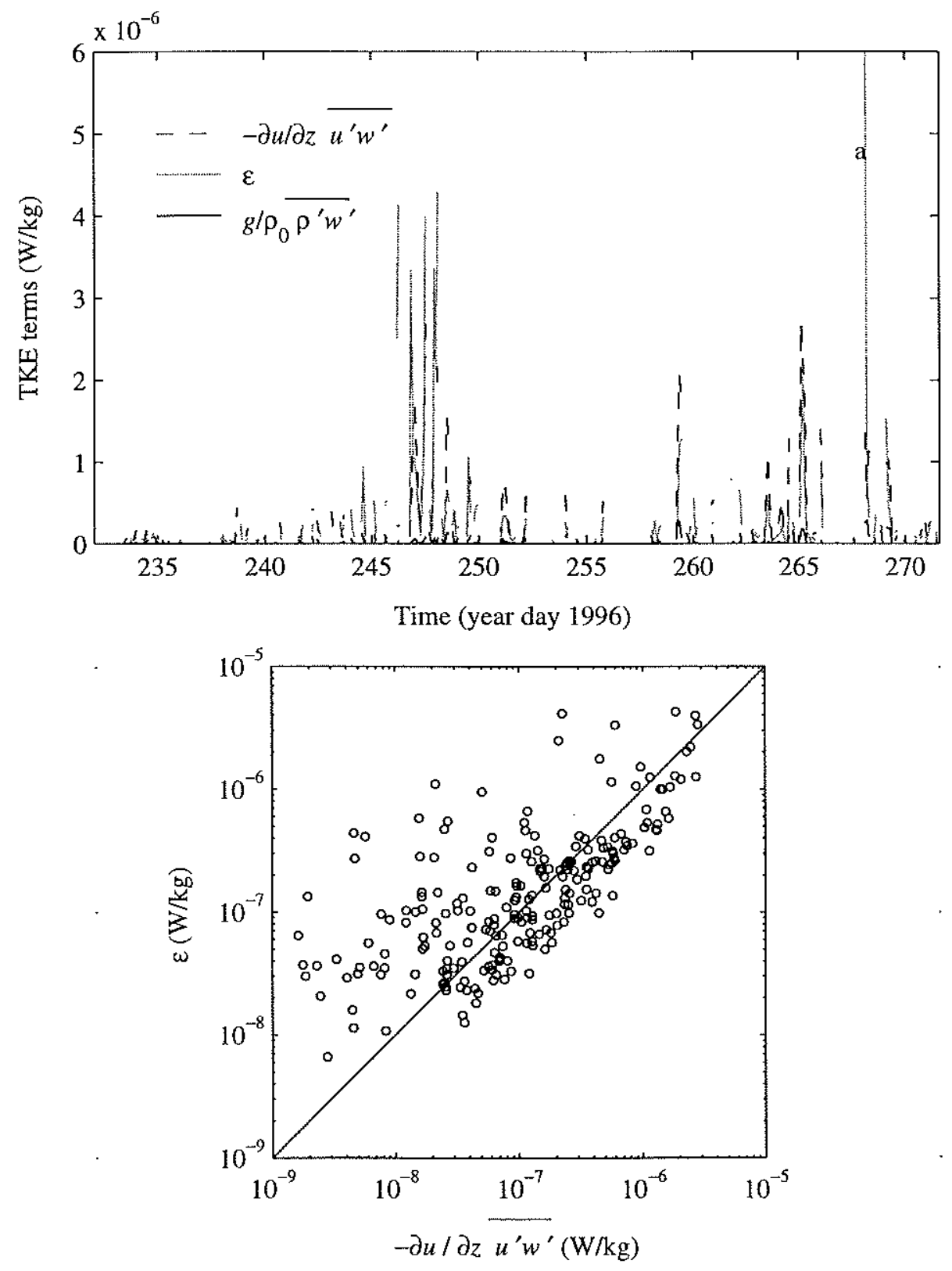

Figure 4.9: Time series (a) and scatter plot (b) of the production and dissipation terms in the TKE budget estimated at a height of $3.8 \mathrm{~m}$ above the bed. Gaps in the time series are the result of bursts discarded because of flow disturbance. 
time series of production and five times the buoyancy flux at the lower position (Figure $4.10 \mathrm{~b}$ ) indicates than the flux Richardson number only approaches the commonly cited critical value of 0.2 [e.g., Wyngaard, 1973] during a few brief periods, in particular, during the period of strong stratification centered on day 255 (Figure 5.3a); otherwise, the flux Richardson number is less than the critical value by upwards of an order of magnitude. For the majority of the time, therefore, the buoyancy flux is not providing a strong constraint on the turbulence dynamics at a height of $0.9 \mathrm{~m}$ above the bed. In contrast, the corresponding time series at the upper position shows that the flux Richardson number tends to maintain a critical value of 0.2 at sub-tidal scales, indicating that closer to the top of the boundary layer the buoyancy flux plays an important role in the turbulence dynamics by acting as a significant sink of TKE. Because of gaps in the time series caused by flow disturbances, it is not possible to evaluate if the flux Richardson number is constant on tidal time scales.

\subsubsection{TSV budget}

There is a large dynamic range in the estimates of sound speed production and dissipation. Time series of the near bottom estimates (Figure 4.12a) are dominated by three heavily stratified periods (Figure 5.3b) when the TSV terms attain peak values of greater than $10^{-4} \mathrm{~m}^{2} \mathrm{~s}^{-3}$. A scatter plot of dissipation vs productions indicates a similar noise floor exists in the $N$ estimates as observed in $\epsilon$ estimates, except that it is approximately an order of magnitude larger, which is not surprising considering the difficulty of making the absolute travel-time measurement [Shaw et al., 1996]. As in the case of the TKE budget, quantitative comparisons are made in terms of log-transformed variables and, to avoid biasing statistics with data points that lie in the dissipation noise floor, comparisons are limited to values 

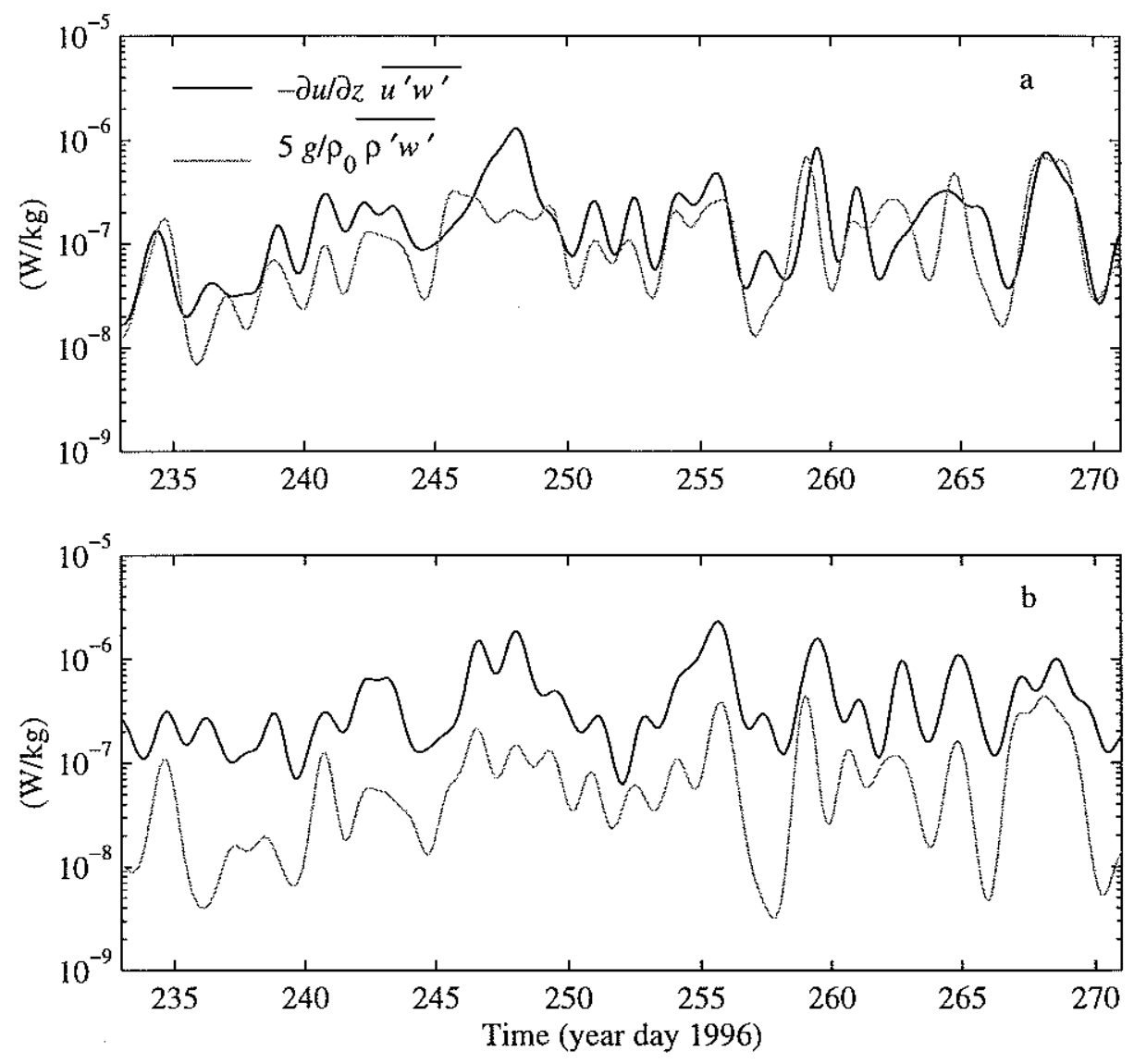

Figure 4.10: Time series of low-pass filtered production of TKE and five times buoyancy flux estimated at $3.8 \mathrm{~m}$ above the bottom (a) and $0.9 \mathrm{~m}$ above the bottom (b). 
of production and dissipation greater than $10^{-6} \mathrm{~m}^{2} \mathrm{~s}^{3}$. There is a good correlation between the production and dissipation $\left(r^{2}=0.60\right.$ for 40 data points). Surprisingly, dissipation is $2.49 \times \div 1.33$ times production above the noise floor.

Time series and a scatter plot of the upper estimates of sound speed production and dissipation (Figure 4.12) indicate that the magnitudes are, in general, larger than those obtained near the bottom. The estimates are very well correlated $\left(r^{2}=0.55\right.$, for 39 data points) and, in contrast to the estimates at $0.9 \mathrm{~m}$ above the bottom, dissipation is $0.45 \times$ $\div 1.44$ larger than production at $3.8 \mathrm{~m}$ above the bottom. A few data points corresponding to large values of dissipation fall well above the one-to-one line and may be indicative of flow disturbances affecting the $N$ estimates. The vertical structure of the production and dissipation estimates suggests that the TSV budget is not balanced locally and does not satisfy wall-layer scaling.

\subsection{Discussion}

The application of the inertial subrange technique to this data set contains an unavoidable difficulty associated with the presence of a noise level that is not small in comparison to the level of the inertial subrange spectra. We have found that the error function involved in the two-parameter estimation problem does not have a well-defined minimum and that small changes in the model can lead to large changes in the estimates of the noise floor and the constant that sets the level of the inertial subrange. For example, we observed a factor of two variability in the dissipation estimates as the theoretical models we employed were refined with particular sensitivity to the accuracy with which we described spatial 

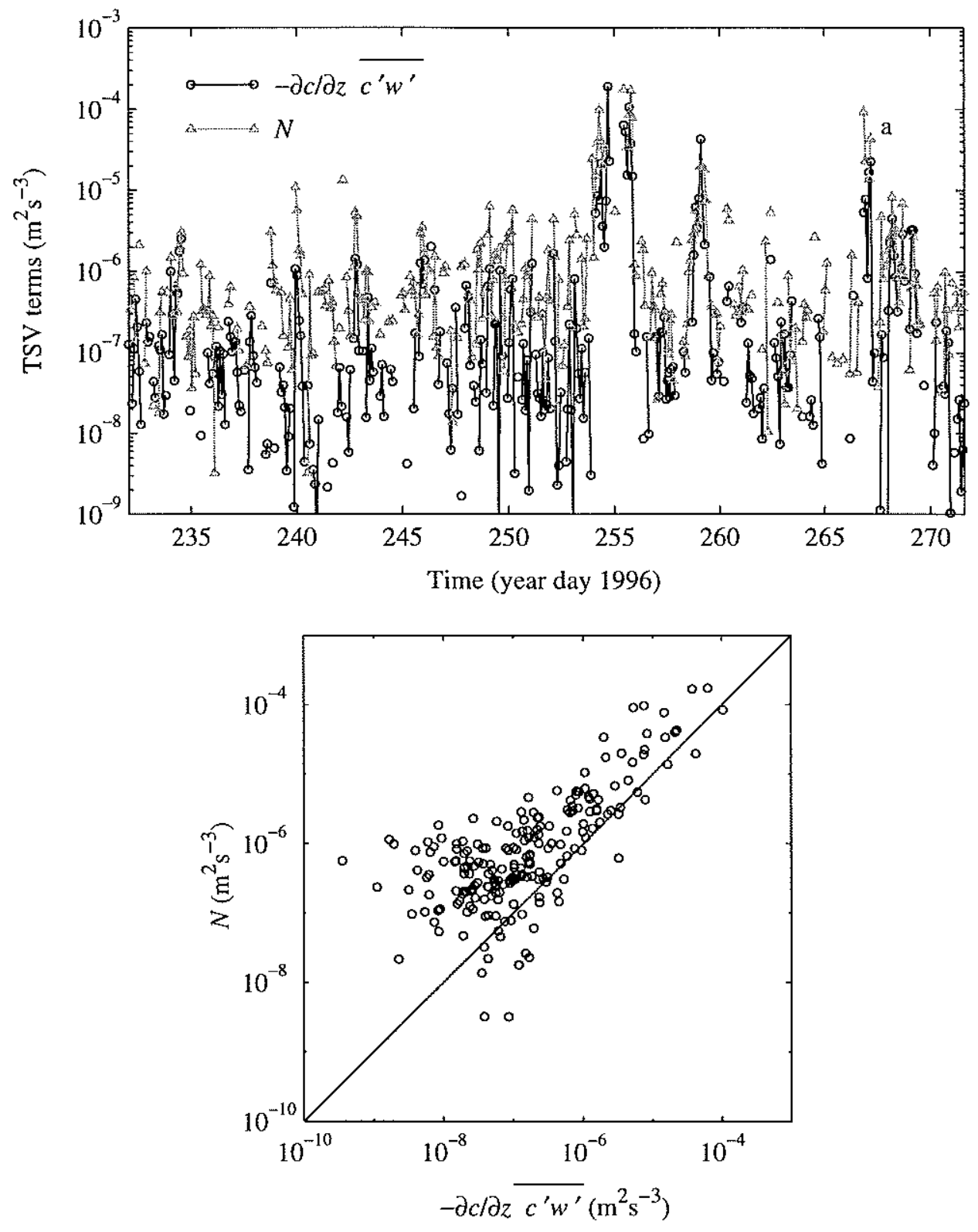

Figure 4.11: Time series (a) and scatter plot (b) of the production and dissipation terms in the TSV budget estimated at a height of $0.9 \mathrm{~m}$ above the bed. 

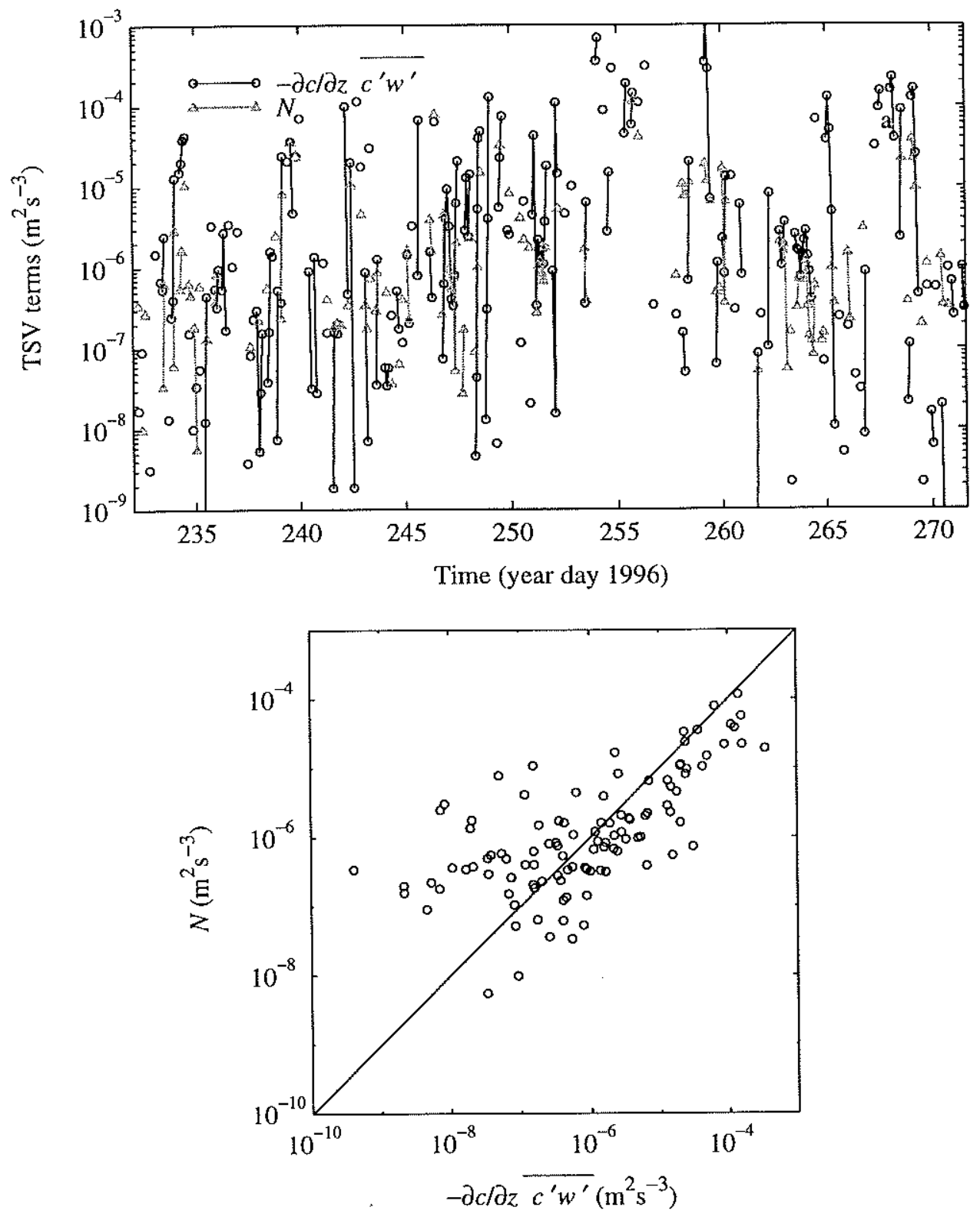

Figure 4.12: Time series (a) and scatter plot (b) of the production and dissipation terms in the TSV budget estimated at a height of $4.35 \mathrm{~m}$ above the bed. 
filtering. The fact that the dissipation estimates converged towards agreement with the production estimates is reassuring and suggests that the detailed modeling of the inertial subrange spectra is worthwhile, i.e. it leads to dissipation estimates that are closer to reality. Improved dissipation estimates in low energy environments will require low noise floor instruments with accurately known responses to resolve the inertial subrange unambiguously or the use of alternative techniques to estimate dissipation. In particular, it seems that direct measurements of dissipation rates would be useful.

The terms in the TKE and TSV equations are composed of estimates of mean vertical gradients, turbulent fluxes, and dissipation rates which are obtained from different frequency bands in the records of velocity and sound speed and are therefore subject to different measurement problems. Therefore the quantitative comparison of the terms in the TKE budget in Figures 7 and 8 and the TSV budget in Figures 9 and 10 are meaningful and the quantitatively consistent results indicate that the measurements obtained with the BASS sensor are reasonably accurate.

The imbalance of the TSV balance may be the result of physical processes rather than measurement problems. Based on the quantitative closure of the TKE budget to within 30 percent, it seems unlikely that the quantities in the TSV budget could suffer from measurement error of more than a factor of two, since sound speed fluctuations were measured with the same instrument and the estimates were obtained with similar processing techniques, with the caveats that sound speed spectra have higher noise floors and the sound speed fluxes were treated in a slightly different way. The most likely physical explanation for the observed TSV balance is the flux divergence term in (4.2), which has been neglected in the assumed local balances, is significant. The observations that production estimates are 
greater than dissipation estimates at the upper location and that the opposite is true at the lower position suggest that there is a divergence of flux at the position of the upper estimates and a convergence at the position of the near-bottom estimates. We also note that, contrary to wall-layer predictions, the production and dissipation estimates increase with height above the bottom. These results are in contrast to those obtained in the wall region of atmospheric boundary layer where the TSV budgets follow wall layer scaling and are in local balance. However, stratification in the atmospheric and oceanic bottom boundary layers results from different underlying processes. In the atmospheric case, thermal forcing from the boundary is dominant, while in the bottom boundary layer case, the source of buoyancy is likely to be due to horizontal advection or mixed layer entrainment. We note in particular, that in the boundary-forced case the regions of production of TKE and TSV are both concentrated near the wall, while in the case of mixed layer entrainment, the region of TSV production is likely to be concentrated in the upper part of the mixed layer where the gradients are large.

The divergence of sound speed flux is a difficult quantity to estimate, requiring differences of a third order quantity which is very susceptible to surface and internal wave contamination. We have attempted to estimate the turbulent flux of sound speed variance, $\overline{c^{\prime 2} w^{\prime}}$, using time derivatives in a manner analogous to the estimation of $\overline{c^{\prime} w^{\prime}}$ discussed in Section 4.2.4. The mean of the estimates of $\overline{c^{\prime 2} w^{\prime}}$ over the entire six-week long deployment has the sign of a downward flux of sound speed variance and the geometric mean of the $\overline{c^{\prime 2} w^{\prime}}$ estimates has the vertical structure required to produce a divergence at the upper two sensors and a convergence at the lower two sensors (Figure 4.13). Additionally, the vertical gradient of the mean vertical profile of $\overline{c^{\prime 2} w^{\prime}}$, approximately $10^{-6} \mathrm{~m}^{2} \mathrm{~s}^{-3}$ at $0.9 \mathrm{~m}$ above the 


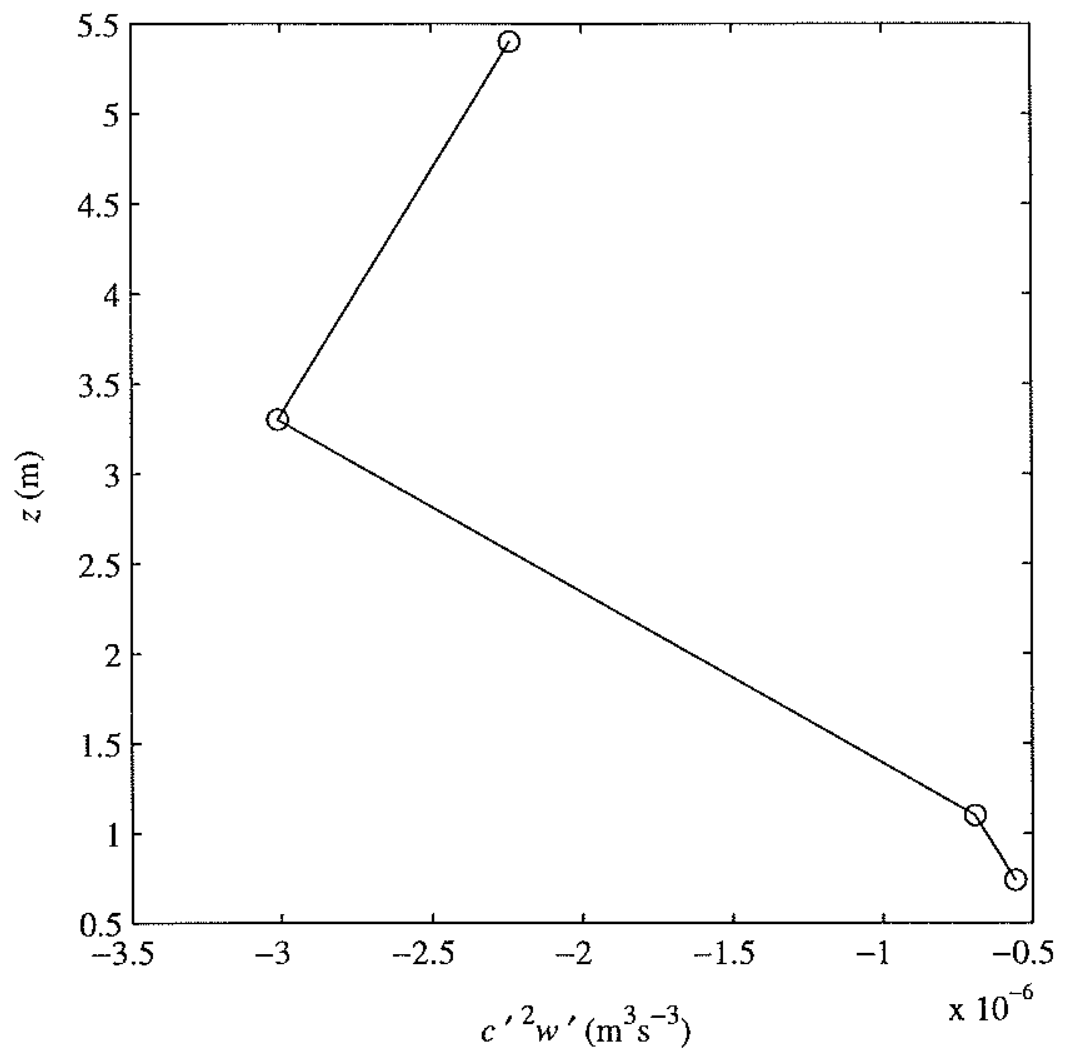

Figure 4.13: Mean vertical profile of the estimates of $\overline{c^{\prime 2} w^{\prime}}$ over the course of the six-week long first deployment at the heights of the four reliable BASS sensors.

bottom, has roughly the order of magnitude required to close the TSV budget.

In oceanic microstructure studies, directly measured dissipation rates are often used to infer turbulent diffusivities based on assumptions regarding the TKE and TSV budgets. The results of this analysis indicate that the assumptions are not always valid and may lead to systematic errors in the estimation of diffusivity. In the Osborn model [Osborn, 1980], the diffusivity of mass is inferred from measurements of $\epsilon$ assuming that the TKE budget is in a local balance between production, buoyancy flux and dissipation and that the flux Richardson number is maintained at a critical value. The present measurements support 
the notion of local balance but are not consistent with the assumption of a constant flux Richardson number, at least in a vicinity close to the boundary. In the Osborn-Cox model [Osborn and Cox, 1972], the diffusivity of scalar quantities is inferred from measurements of $N$ assuming that the TSV budget is balanced locally. The present measurement are not consistent with the assumption of a local balance in the TSV budget.

\subsection{Summary and Conclusions}

In this paper, a simplified TKE budget, in which production is balanced by dissipation and buoyancy flux, and a simplified TSV balance, in which production equals dissipation, have been tested. The calculations are based on six-week long records of velocity and sound speed measured between 0.7 and $5.4 \mathrm{~m}$ above bottom on the New England shelf. Estimates of the required fluxes and gradients are direct, while estimates of dissipation rates are indirectly. obtained from inertial subrange spectra. Estimates of dissipation rates require consideration of spatial filtering due to a finite sensor volume, temporal aliasing, and instrument noise.

The results of the analysis indicate the following conclusions. In the TKE budget, production and dissipation are correlated $\left(r^{2}=0.33\right.$ at $0.9 \mathrm{~m}$ above the bottom and 0.38 at $3.8 \mathrm{~m}$ above the bottom) and roughly consistent in magnitude (regression coefficient = $0.79 \times \div 1.10$ at $0.9 \mathrm{~m}$ above bottom and $0.90 \times \div 1.19$ at $3.8 \mathrm{~m}$ above bottom). Buoyancy flux is not a significant component of the TKE budget at $0.9 \mathrm{~m}$ above the bottom except during a few events, while at $3.8 \mathrm{~m}$ above the bottom the flux Richardson number maintains a roughly constant value of 0.2 . In the TSV budget, production and dissipation are well-correlated $\left(r^{2}=0.60\right.$ at $0.9 \mathrm{~m}$ above the bottom and 0.55 at $3.8 \mathrm{~m}$ above the bottom $)$, but dissipation is 
$2.49 \times \div 1.33$ times production at $0.9 \mathrm{~m}$ above the bottom while dissipation is $0.45 \times \div 1.44$ times dissipation at $3.8 \mathrm{~m}$ above the bottom. The imbalance between production and dissipation in the TSV budget is likely due to the divergence of the vertical flux of sound speed variance, a quantity that is difficult to estimate, although the measurements indicate that this term has the correct sign and roughly the magnitude required to close the budget. The absence of a critical flux Richardson number at $0.9 \mathrm{~m}$ above the bottom and the imbalance of the TSV budget at both heights imply inaccuracies in the use of the Osborn and Osborn-Cox models to infer turbulent diffusivities from direct dissipation rate estimates in the bottom boundary layer. 


\section{Bibliography}

G. K. Batchelor. The Theory of Homogeneous Turbulence. Cambridge University Press, London, 1951.

T. H. Ellison. Turbulent transport of heat and momentum from an infinite rough plate. $J$. Fluid Mech., 2:456-466, 1957.

M. Fleury and R. G. Lueck. Direct heat flux estimates using a towed vehicle. J. Phys. Oceanog., 24:801-818, 1994.

J. J. Fredericks, J. H. Trowbridge, A. J. Williams, G. Voulgaris, and W. J. Shaw. Fluid mechanical measurements within the bottom boundary layer during coastal mixing and optics. Technical report, Woods Hole Oceanographic Institution, 1999.

N. Galbraith, W. Ostrom, B. Way, S. Lentz, S. Anderson, M. Baumgartner, A. Plueddemann, and J. Edson. Coastal mixing and optics experiment: Mooring deployment cruise report. Technical report, WHOI, 1997.

A. E. Gargett and J. N. Moum. Mixing efficiencies in turbulent tidal fronts: Results from direct and indirect measurements of density flux. J. Phys. Oceanog., 25:2583-2608, 1995. 
T. F. Gross and A. R. M. Nowell. Spectral scaling in a tidal boundary layer. J. Phys. Oceanogr., 15:496-508, 1985.

U Hogstrom. Review of some basic characteristics of the atmospheric surface layer. Bound.Layer Meteor., 78:215-246, 1996.

J. C. Kaimal and J. E. Gaynor. Another look at sonic anemometry. Bound.-Layer Meteor., $56: 563-589,1991$.

J. C. Kaimal, J. C. Wyngaard, and D. A. Haugen. Deriving power spectra from a threecomponent sonic anemometer. J. Appl. Meteor., 7:827-837, 1968.

J. C. Kaimal, J. C. Wyngaard, Y. Izumi, and Cote O. R. Spectral characteristics of surfacelayer turbulence. Quart. J. R. Met. Soc., 98:563-589, 1972.

K. Kawanisi and S. Yokosi. Mean and turbulence characteristics in a tidal river. Est. Coast. Shelf Sci., 38:447-469, 1994.

S. E. Larsen, J. B. Edson, C. W. Fairall, and P. G. Mestayer. Measurement of temperature spectra by a sonic anemometer. J. Atmos. and Ocean. Tech., 10:345-354, 1993.

R. Limeburner. Code-2: Moored array and large-scale data report. Technical report, Woods Hole Oceanographic Institution, 1985.

J. L. Lumley and E. A. Terray. Kinematics of turbulence convected by a random wave field. J. Phys. Oceanog., 13:2000-2007, 1983.

M. G. McPhee. Turbulent heat flux in the upper ocean under sea ice. J. Geophys. Res., 97: $536-5379,1992$. 
J. N. Moum. The quest for $k_{\rho}$ - preliminary results from direct measurements of turbulent fluxes in the ocean. J. Phys. Oceanog., 20:1980-1984, 1990.

T. R. Osborn. Estimates of the local rate of vertical diffusion from dissipation measurements. J. Phys. Oceanogr., 10:83-89, 1980.

T. R. Osborn and C. S. Cox. Oceanic fine structure. Geophys. Fluid Dyn., 3:321-345, 1972.

T. B. Sanford and R.-C. Lien. Turbulent properties in a homogeneous tidal bottom boundary layer. J. Geophys. Res., 104:1245-1257, 1999.

W. J. Shaw and J. H. Trowbridge. The measurement of near-bottom turbulent fluxes in the presence of energetic wave motions. J. Atm. and Ocean. Tech., submitted.

W. J. Shaw, A. J. Williams, and J. H. Trowbridge. Measurement of turbulent sound speed fluctuations with an acoustic current meter. In Oceans96. MTS/IEEE, 1996.

B. A. Silverman. The effect of spatial filtering on spectrum estimation. J. Appl. Meteor., $7: 168-172,1968$.

H. Tennekes and J. L. Lumley. A First Course in Turbulence. MIT Press, Cambridge, Mass., 1972.

D. A. Trivett. Diffuse Flow from Hydrothermal Vents. PhD thesis, WHOI/MIT, 1991.

J. H. Trowbridge, W. R. Geyer, M. M. Bowen, and A. J. Williams. Near-bottom turbulence mesurements in a partially mixed estuary: Turbulent energy balance, velocity structure, and along-channel momentum balance. J. Phys. Oceanogr., in press. 
D. C. Twichell, C. E. McClellan, and B. Butman. Morphology and processes associated with the accumulation of the fine-grained sediment deposit on the southern new england shelf. J. Sed. Pet., 51:269-280, 1981.

A. J. Williams, J. S. Tochko, R. L. Koehler, W. D. Grant, Gross T. F., and C. V. R Dunn. Measurement of turbulence in the oceanic bottom boundary layer with an acoustic current meter array. J. Atm. and Ocean. Tech., 4:312-327, 1987.

J. C. Wyngaard. On surface layer turbulence. In D. A. Haugen, editor, Workshop on Micrometeorology, pages 101-149. Amer. Meteor. Soc., 1973.

J. C. Wyngaard. Atmospheric turbulence. Ann. Rev. Fluid Mech., 24:205-233, 1992.

J. C. Wyngaard and O. R. Cote. The budgets of turbulent kinetic energy and temperature variance in the atmospheric surface layer. J. Atmos. Sci, 28:190-201, 1971.

H. Yamazaki and T. Osborn. Dissipation estimates for stratified turbulence. J. Geophys. Res., 95:9379-9744, 1990. 


\title{
Chapter 5
}

\section{Flux-Profile Relationships in the Continental Shelf Bottom Boundary Layer}

\author{
William J. Shaw and John H. Trowbridge
}

(This chapter represents a manucript that is to be submitted to The Journal of Geophysical Research.)

\begin{abstract}
Dimensionless profiles of velocity and a scalar (speed of sound) are computed from direct measurement of the required fluxes and vertical gradients obtained at heights of 1 to $4 \mathrm{~m}$ above the bed in the bottom boundary layer (BBL) of the New England shelf. The results are presented in terms of a local similarity theory based on the height above the bed, the Monin-Obukhov (MO) length and the height of the mixed layer. The MO length is estimated from the measured fluxes and an empirical sound speed-density relationship and the height of the mixed layer is taken as the Zilitinkevich scale. The forcing at the site is predominantly tidal and the source of the buoyancy flux is horizontal advection of stratification, mixed layer entrainment and, during large storms, sediment resuspension. Within $1 \mathrm{~m}$ of the bottom, the Prandtl-Karman law of the wall is satisfied during nearly neutral conditions, and the dependence of the dimensionless shear on the MO stability parameter is quantitatively consistent with atmospheric results for values of the stability parameter up to approximately one. In contrast, at measurement heights greater than 1 $\mathrm{m}$ above the bed, the law of the wall is rarely, if ever, satisfied, although the dependence of the dimensionless shear is qualitatively consistent with previous atmospheric results. Measurements of boundary layer height are fairly well estimated by the Zilitinkevich mixed layer height scale. The dimensionless gradients and the turbulent Prandtl number are dependent on the ratio of height above bottom and the mixed layer scale. The results indicate that the height of the mixed layer imposes a constraint on the Prandtl mixing length.
\end{abstract}




\subsection{Introduction}

Establishing turbulence closure hypotheses is the primary goal of turbulent boundary layer research and a significant body of knowledge has been obtained from semi-empirical theory and observations of laboratory, engineering and geophysical boundary layer flows. The turbulence closure problem in the continental shelf bottom boundary layer (BBL) is relevant because the $\mathrm{BBL}$ is an important source of mixing, a sink of momentum and a conduit for cross-shelf exchange. The dynamics of coastal ocean BBL's are complicated by the large number of possible forcing mechanisms, including tides, wind and density differences, and the presence of stable stratification. The significance and complexity of the continental shelf BBL combine to make turbulence closure an important observational problem in coastal oceanography.

In spite of its importance, detailed observational studies of BBL turbulence have been rare owing to the technical challenges involved in obtaining the measurements. Of the few studies that have obtained direct measurements of turbulent shear stress, many have been limited to the determination of values for the bottom drag coefficient [e.g., Heathershaw, 1979]. Drag laws are commonly used as closures in models, but the drag coefficient is an integrated quantity and its value is therefore dependent on the bottom roughness which varies with bottom type and flow conditions [Monin and Yaglom, 1971]. Although numerous studies have estimated bottom shear stress by assuming that the Prandtl-Karman law of the wall (see Section 5.2) was satisfied [e.g., Charnock, 1959, Sternberg, 1968, Gross and Nowell, 1983, Lueck and Lu, 1997], only a small subset of the studies that obtained direct shear stress measurements included the measurement of the mean shear necessary to test 
the law of the wall. Gross and Nowell [1983] and Sanford and Lien [1999] have reported good agreement between bottom stress estimated both directly and with the log-profile technique in tidal channels, indicating that law of the wall is satisfied close to the bottom. Smith and McLean [1977] and Soulsby and Dyer [1979] have tried to explain departures from the law of the wall in terms of the effects of form drag and accelerations, respectively. Trowbridge et al. [in press] reports deviations from the law of the wall in a tidal estuary that are inconsistent with local stratification and are attributed to the presence of a strong pycnocline above the bottom mixed layer. Stacey et al. [submitted] report measurements of eddy diffusivity for momentum that are compared with results from a second-order turbulence closure model. All of the oceanic studies in which direct measurements of stress and shear were obtained have been limited to strong tidal flows and, with the exception of the studies by Sanford and Lien [1999] and Stacey et al. [submitted], the studies have been limited to the lowest few meters of the boundary layer. To date, a single attempt to measure scalar fluxes in the bottom boundary layer has been reported by Kawanisi and Yokosi [1994], who measured the turbulent salt flux in a tidal estuary.

In studying the oceanic bottom boundary layer, we can also draw on knowledge obtained in other boundary layer flows. The atmospheric boundary layer is a particularly attractive source because it is a reasonable analog for the oceanic bottom boundary layer and because it has been intensively studied over the last 30 years. There are important differences between the atmospheric boundary layer and the oceanic bottom boundary layer. The buoyancy forcing in the atmospheric case is dominated by the surface heat flux. In the oceanic bottom boundary layer, the surface buoyancy flux is nearly zero, except when sediment resuspension occurs. Thermo-haline induced buoyancy flux in the oceanic case 
must be supplied by the horizontal advection of stratification or mixed layer entrainment. Another important distinction is the difference in spatial scale. Assuming the height of the boundary layer is proportional to $u_{*}$, the atmospheric boundary layer will be larger than the oceanic boundary layer by the factor of $\sqrt{\rho_{\text {water }} / \rho_{\text {air }}} \simeq 30$ for the same surface stress. For example a $1 \mathrm{~km}$ thick atmospheric boundary layer with a $100 \mathrm{~m}$ constant flux layer would roughly correspond to a $30 \mathrm{~m}$ thick oceanic bottom boundary layer with a $3 \mathrm{~m}$ thick constant stress layer.

In this paper, we present dimensionless profiles of velocity and sound speed that are computed from direct measurements of the required fluxes and vertical gradients obtained in the bottom boundary layer of the New England shelf at heights of 1 to $4 \mathrm{~m}$ above the bottom. The results are presented in terms of two dimensionless parameters, the ratio of height above bottom and the Monin-Obukhov (MO) length and the ratio of the height above bottom and the height of the mixed layer. Our goals are to determine the dependence of the turbulent length scale, taken as the Prandtl mixing length, on local stratification and on the height of the boundary layer and to determine if the turbulent diffusivities for momentum and scalars are equal.

The remainder of the paper is organized into the following sections: a presentation of the similarity theory in which we present our results (Section 5.2), a description of the methods used to estimate the quantities required for the theory (Section 5.3), a presentation and discussion of the results (Sections 5.4 and 5.5), and a summary and conclusions (Section 5.6). 


\subsection{Similarity Theory}

We consider a boundary layer that mixes momentum and density. We assume that turbulent fluctuations of density are small and are linearly related to a measurable scalar. On physical grounds, we assume that the dynamically significant independent variables in the BBL come from the set

$$
\left(\overline{u^{\prime} w^{\prime}}, \overline{c^{\prime} w^{\prime}}, \frac{d \rho}{d c} \frac{g}{\rho_{0}}, z, \delta\right)
$$

Here, $\overline{u^{\prime} w^{\prime}}$ is the kinematic shear stress (the correlation between the turbulent fluctuations of horizontal and vertical velocity). $\overline{c^{\prime} w^{\prime}}$ is the turbulent flux of a scalar $c$ (the correlation between the turbulent fluctuations of the scalar and vertical velocity). Both $\overline{u^{\prime} w^{\prime}}$ and $\overline{c^{\prime} w^{\prime}}$ are local values, i.e. they are functions of $z \cdot \frac{d \rho}{d c}$ is the derivative of density with respect to the scalar, $g / \rho_{0}$ is the buoyancy parameter (the ratio of the gravitational acceleration $g$ to a reference density $\left.\rho_{0}\right), z$ is the height above the bottom and $\delta$ is a scale for the height of the boundary layer. Thus, the structure of the mean flow can be expressed as functions of the set of independent variables. In particular, we may express the mean shear as

$$
\frac{\partial u}{\partial z}=g_{m}\left(\overline{u^{\prime} w^{\prime}}, \overline{c^{\prime} w^{\prime}}, \frac{d \rho}{d c} \frac{g}{\rho_{0}}, z, \delta\right)
$$

and the mean scalar gradient as

$$
\frac{\partial c}{\partial z}=g_{h}\left(\overline{u^{\prime} w^{\prime}}, \overline{c^{\prime} w^{\prime}}, \frac{d \rho}{d c} \frac{g}{\rho_{0}}, z, \delta\right)
$$

The dimensional gradient functions, $g_{m}$ and $g_{h}$ (the subscript $h$ is used in deference to atmospheric tradition where the flux of interest is heat) are seen to represent turbulence 
closure hypotheses for the fluxes of momentum and a scalar, respectively. We can use dimensional analysis to reduce the number of variables in (5.2) and (5.3) from six to three resulting in

$$
\frac{\kappa z}{u_{*}} \frac{\partial u}{\partial z}=\phi_{m}\left(\frac{z}{L}, \frac{z}{\delta}\right)
$$

and

$$
\frac{\kappa z}{c_{*}} \frac{\partial c}{\partial z}=\phi_{h}\left(\frac{z}{L}, \frac{z}{\delta}\right)
$$

where $\phi_{m}$ is the dimensionless shear, $\phi_{h}$ is the dimensionless sound speed gradient and $\kappa$ is von Karman's constant. The shear velocity $u_{*}$, a characteristic scalar fluctuation $c_{*}$ and the Monin-Obukhov length $L$ are defined as

$$
\begin{gathered}
u_{*}=\left.\overline{\mid u^{\prime} w^{\prime}}\right|^{1 / 2}, \\
c_{*}=\overline{\overline{-c^{\prime} w^{\prime}}} \\
u_{*}
\end{gathered}
$$

and

$$
L=\frac{u_{*}^{3}}{\kappa \frac{g}{\rho_{0}} \frac{d \rho}{d c} \overline{c^{\prime} w^{\prime}}}
$$

respectively. As this similarity theory does not include length scales representing the bottom roughness or accelerations, it is limited to heights above bottom much greater than the roughness length [Monin and Yaglom, 1971] and to quasi-steady conditions.

The dimensionless shear $\phi_{m}$ may be regarded as the ratio of $z$ to a momentum mixing 
length. The mixing length is defined as

$$
l_{m}=\frac{u_{*}}{\kappa \frac{\partial u}{\partial z}}
$$

An analogous mixing length may be defined for scalars as

$$
l_{h}=\frac{c_{*}}{\kappa \frac{\partial c}{\partial z}}
$$

In essence, the dimensionless gradients provide a framework for evaluating the variability in $z / l_{m}$ and $z / l_{h}$ as a function of the relative importance of mechanical and thermal forcing, represented by the stability parameter $z / L$, and proximity to the edge of the boundary layer, represented by $z / \delta$.

The turbulent diffusivities for momentum and scalars may be related to $\phi_{m}$ and $\phi_{h}$ as

$$
k_{m}=\frac{\kappa z u_{*}}{\phi_{m}}
$$

and

$$
k_{h}=\frac{\kappa z u_{*}}{\phi_{h}}
$$

respectively. And, therefore, the turbulent Prandtl number may be written as

$$
\operatorname{Pr}_{t} \equiv \frac{k_{m}}{k_{h}}=\frac{\phi_{h}}{\phi_{m}}
$$




\subsubsection{Monin-Obukhov Similarity}

In the surface (wall) layer of the atmospheric boundary layer $(z / \delta<0.1$ ), the fluxes of momentum and heat are nearly equal to the values at the surface [Monin and Yaglom, 1971] and $\phi_{m}$ and $\phi_{h}$ are expected to be dependent only on $z / L$. This is the backbone of surface layer MO similarity theory [e.g., Wyngaard, 1973]. MO similarity has been confirmed in the lowest $10-20 \mathrm{~m}$ of the atmospheric boundary layer by careful measurement of the required fluxes and vertical gradients. In particular, empirical forms for $\phi_{m}$ and $\phi_{h}$ were determined from measurements obtained during the landmark Kansas Mast experiment [Businger et al., 1971]. More recently, it has been proposed that MO scaling can be applied to the outer part $(z / \delta>0.1)$ of the nocturnal (stable) atmospheric boundary layer using local values of the fluxes [Nieuwstadt, 1984, Sorbjan, 1986]. We expect that local scaling may be appropriate in the oceanic bottom boundary layer.

For near-neutral conditions $(z / L<<1)$, the similarity relationships (5.4) and (5.5) predict that the dimensionless gradients reduce to constants. In particular, von Karman's constant has been defined to make

$$
\frac{\kappa z}{u *_{b}} \frac{\partial u}{\partial z}=1
$$

near the wall for neutral conditions, where $u_{* b}$ is the bottom shear velocity. (5.14) has come to be known as the Karman-Prandtl law of the wall and it is expected to hold in all wall-bounded shear flows for a limited range of heights. 


\subsubsection{Boundary Layer Height Scaling}

The effect of the parameter $z / \delta$ on dimensionless gradients is more poorly understood than the effect of stratification. Part of the problem is the difficulty of defining a suitable measure of boundary layer height [e.g., Monin and Yaglom, 1971]. Laboratory studies of neutral boundary layer flows on flat plates indicate that the height of the turbulent part of the flow is very unsteady, varying from $0.3-0.4 \delta$ to $1.2 \delta$ (where $\delta$ is defined as the height at which $u=0.99 u_{\infty}$ ) [Monin and Yaglom, 1971]. Further, in stably stratified boundary layer flows, the stratification is expected to play an important role in setting the height of the boundary layer [e.g., Thompson, 1973].

Coles [1956] has proposed a universal form for the outer part $(z / \delta$ significant $)$ of neutral laboratory boundary layers based on a combination of a logarithmic velocity profile and a wake function which is dependent on $z / \delta$. The wake function gives the velocity profile at a point of separation, $u_{* b}=0$ and predicts an increase in shear from a logarithmic velocity profile in the outer part of the boundary layer.

Also of importance, we require a scale for the boundary layer height in the presence of overlying stable stratification. This is taken as the Zilitinkevich [1972] length scale defined here as

$$
\delta_{Z}=\frac{u_{*}}{\sqrt{(\omega-f) N_{b \infty}}}
$$

where $(\omega-f)$, the difference between the semi-diurnal and inertial frequencies, provides the appropriate time scale for a rotary tidal boundary layer [Soulsby, 1983] and $N_{b \infty}$ is the interior buoyancy frequency. Thompson [1973] has shown that $\delta_{Z}$ is proportional to the height of a boundary layer governed by a bulk Richardson criterion for the case of steady 
forcing with a proportionality constant close to one.

\subsection{Methods}

\subsubsection{Measurements}

The measurements used in the analysis were collected as part of the bottom boundary layer tripod component of the Coastal Mixing and Optics (CMO) experiment during a six-week long period beginning on Aug. 17, 1996. The field site is located approximately $100 \mathrm{~km}$ south of Martha's Vineyard, MA in $70 \mathrm{~m}$ of water on the New England shelf.

The instrumentation deployed on the bottom tripod has been described by Fredericks et al. [1999] and Shaw et al. [submitted]. The most important instrumentation for the purposes of this paper was a vertical array of seven Benthic Acoustic Stress Sensors (BASS)'s [Williams et al., 1987] that measured the mean and fluctuating components of the threedimensional velocity vector and the speed of sound at heights of $0.4,0.7,1.1,2.2,3.3$, 5.4 and $7 \mathrm{~m}$ above the bottom. The BASS sensors were sampled at $1.2 \mathrm{~Hz}$ in 27 minute bursts in two hours cycles consisting of three half-hour periods in which data was obtained, followed by a half-hour period in which the instruments were idle. In addition to the bottom tripod, an array of moorings that measured velocity, temperature, and conductivity throughout the water column was deployed by Woods Hole Oceanographic Insitution investigators [Galbraith et al., 1997]. The central mooring of the WHOI array was located approximately $200 \mathrm{~m}$ from the bottom tripod. Also, an additional bottom tripod outfitted with optical sensors including a trasmissometer [Dickey et al., submitted] was deployed near the BASS. 


\subsubsection{Boundary Layer Estimates}

The similarity theory outlined in Section 5.2 requires measurements of the fluxes of momentum and a scalar, the vertical gradients of velocity and the scalar, the relationship between the scalar and density, and the height of the boundary layer. The scalar used here is sound speed.

We calculated direct estimates of the vertical fluxes of momentum and sound speed from half hour records of velocity and sound speed that were Reynolds decomposed into mean and fluctuating components. We used a linear filtration and differencing technique [Shaw and Trowbridge, submitted] to overcome the problem of surface wave contamination of the flux estimates. The sound speed flux estimates also suffered from internal wave contamination that we removed with a high-pass filter, although an empirical correction was required to account for turbulent covariance that was lost in the filtering process [Shaw et al., submitted]. The dimensionless gradients were calculated using local values of the fluxes and, for the case of the dimensionless shear $\phi_{m}$, the gradient was calculated in the down-stress direction. The required sound speed-density relationship [Shaw and Trowbridge, submitted] was obtained from the near-bottom measurements of the WHOI central mooring.

In the course of the analysis, we encountered a few flow disturbance and instrumentation problems that have been previously described [Shaw et al., submitted]. Bursts that were disturbed by turbulent wakes shed from the tripod legs were discarded. Data from the top sensor was discarded because of severe disturbance due to the tripod legs and electronic problems. Mean velocity data at sensor $1(0.4 \mathrm{~m}$ above bottom) and sensor $5(3.3 \mathrm{~m}$ above the bottom) were judged to have suffered from zero offset drift and were not used 
for estimating gradients. Of the remaining four sensors, we calculated vertical gradients of velocity and sound speed as first order differences between adjacent heights, corresponding to estimates at $0.9,1.65$ and $3.8 \mathrm{~m}$ above the bottom. Accompanying flux estimates at those heights were obtained as averages of the values at adjacent sensors. Estimates from the Reynolds decomposition performed on contiguous half hour bursts were averaged together to yield times series of 476 data points at a two hour time interval.

We obtained two measurements of the height of the bottom boundary layer. The first is a mixed layer height based on the elevation at which the density difference $\rho(z)-\rho(0)$ exceeds a threshold value [e.g., Lentz and Trowbridge, 1991]. We used both the BASS sound speed measurements (converted to density differences with the empirical relationship) and measurements of density from the central WHOI mooring so that the vertical resolution was fairly fine, especially near bottom. We took the threshold density difference as $0.1 \mathrm{~kg} / \mathrm{m}^{3}$. We also estimated the top of the boundary layer in a mechanical sense by extrapolating linear fits to measured profiles of momentum flux to an elevation of zero flux, which was defined as the height of the mechanical boundary layer. The mixed layer height scale was calculated from (5.15) using the buoyancy frequency just above the top of the measured mixed layer and values of $f$ and $\omega$ of $10^{-4}$ and $1.5 \times 10^{-4} \mathrm{rad} / \mathrm{s}$, respectively.

In addition to the buoyancy flux that is caused by thermo-haline stratification, the bottom boundary layer is also subject to a buoyancy flux due to resuspension of sediment particles. We estimated the concentration of suspended sediment in the bottom boundary layer using the optical attenuation data of Dickey et al. [submitted] from the nearby bottom tripod and a calibration obtained from shipboard optical attenuation measurements and a bottom grab sample (J. Blakey, pers. comm.). We further estimated the buoyancy flux 
associated with the suspended sediment by assuming a balance between the turbulent flux of sediment and gravitational settling,

$$
\frac{g}{\rho_{0}}{\overline{\rho^{\prime} w^{\prime}}}_{\text {sed }}=g(s-1) w_{s} C
$$

where $s, w_{s}$ and $C$ are the specific gravity, settling velocity, and volume concentration of the suspended particles, respectively. The values we used are $s=2.65$ and $w_{s}=0.6 \mathrm{~mm} / \mathrm{s}$ (Traykovsky, pers. comm.), corresponding to course silt-sized quartz particles.

\subsection{Results}

\subsubsection{Site Conditions}

The rapid sampling of the BASS sensors over a period of six weeks allowed us to resolve a wide spectrum of velocity and sound speed variability: from sub-tidal motion to the inertial subrange of turbulent eddies. We formed composite rotary spectra of horizontal velocity and shear at $1.1 \mathrm{~m}$ above the bottom (Figure 5.1) by combining the spectra obtained from the Reynolds averaged velocity from each half-hour burst and the averaged spectrum obtained from the concatenation of the two hourly cycle of three nearly consecutive bursts. The dominant forcing in the BBL at the CMO site during the deployment is the polarized semidiurnal tide, visible as the large peak at $-1.5 \times 10^{-4} \mathrm{rad} / \mathrm{s}$ (Figure 5.1a). The next largest forcing is from surface waves visible as the broad unpolarized peak centered on $0.4 \mathrm{rad} / \mathrm{s}$, corresponding to 15 second waves. Also visible in the low-frequency part of the spectrum is a small peak at the inertial frequency and a barely resolved peak at the frequency of the 
diurnal tide. There is a internal wave continuum between the semi-diurnal tidal frequency and the local buoyancy frequency (approximately $10^{-2} \mathrm{rad} / \mathrm{s}$ ). At frequencies greater than the buoyancy, the observed variance is the result of energetic eddies. The shear spectrum is also dominated by the semi-diurnal tide (Figure $5.1 \mathrm{~b}$ ). We see from Figure $5.1 \mathrm{~b}$ that a Reynolds averaging period of one half-hour can be expected to separate the mean shear and the turbulent fluctuations.

As a description of the vertical structure of the mean horizontal velocity at the CMO site, we decompose the horizontal velocity field, represented as $u+i v$, into complex empirical orthogonal modes (EOM)'s [see Davis, 1976]. The lowest EOM accounts for $98 \%$ of the observed variance and therefore provides a useful characterization of the vertical structure. The amplitude of the lowest EOM (Figure 5.2a) contains curvature throughout the range of heights spanned by the measurements. There is little or no veering observed in the EOM over the vertical extent of the measurements (Figure 5.2a).

To further put the turbulence measurement in context, it is useful to describe time series of near-bottom wave orbital velocity, suspended sediment concentration, along-shelf velocity, water column temperature and sound speed (Figure 5.3). During the first deployment, Hurricane Edouard passed within $110 \mathrm{~km}$ of the site on day 246 and Hurricane Hortense passed within $350 \mathrm{~km}$ of the site on day 258 . The passage of the hurricanes is visible as peaks in the time series of near-bottom wave orbital velocity (Figure 5.3a). The energetic wave-induced velocities were also responsible for the resuspension of sediment that reached concentrations of greater than $10 \mathrm{mg} / 1$ (Figure $5.3 \mathrm{a}$ ). The bottom boundary layer response to both hurricanes was a strong westward mean flow (Figure 5.3b) that generated enough mixing to warm the bottom boundary layer by several deg $\mathrm{C}$ in each case (Figure $5.3 \mathrm{c}$ ). In 

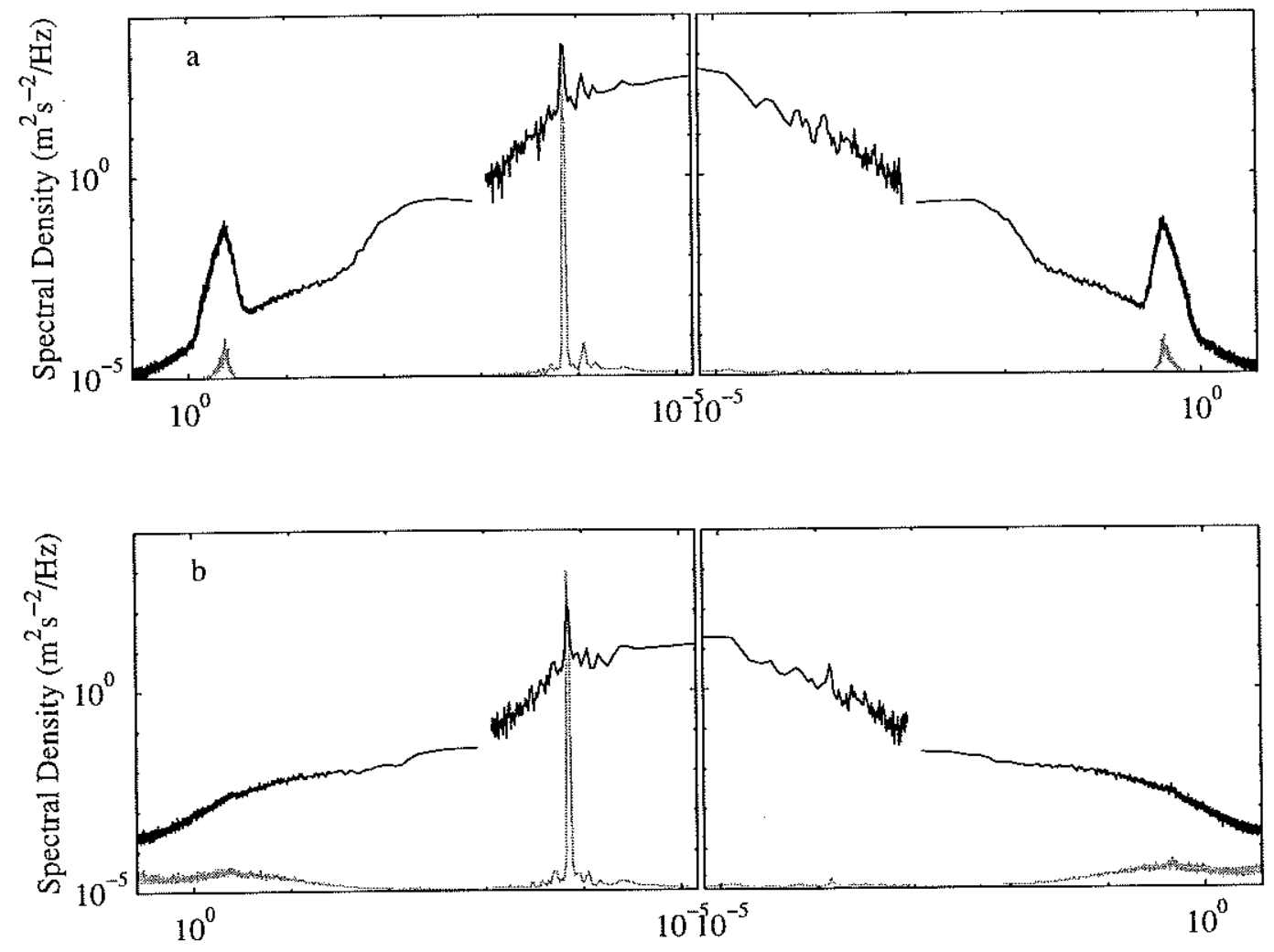

Frequency $(\mathrm{rad} / \mathrm{s})$

Figure 5.1: Rotary spectra of horizontal velocity (a) and horizontal shear (b) measured by the BASS sensor at $1.1 \mathrm{~m}$ above the bottom. The left-hand panels correspond to negative (clockwise) motions and the right-hand panels correspond to positive (counterclockwise motion) motion. The dark lines represent logarithmic spectra corresponding to the scales on the left and the gray lines represent the same spectra plotted in energy-preserving form (frequency times spectral density on semilogarithmic axes). 

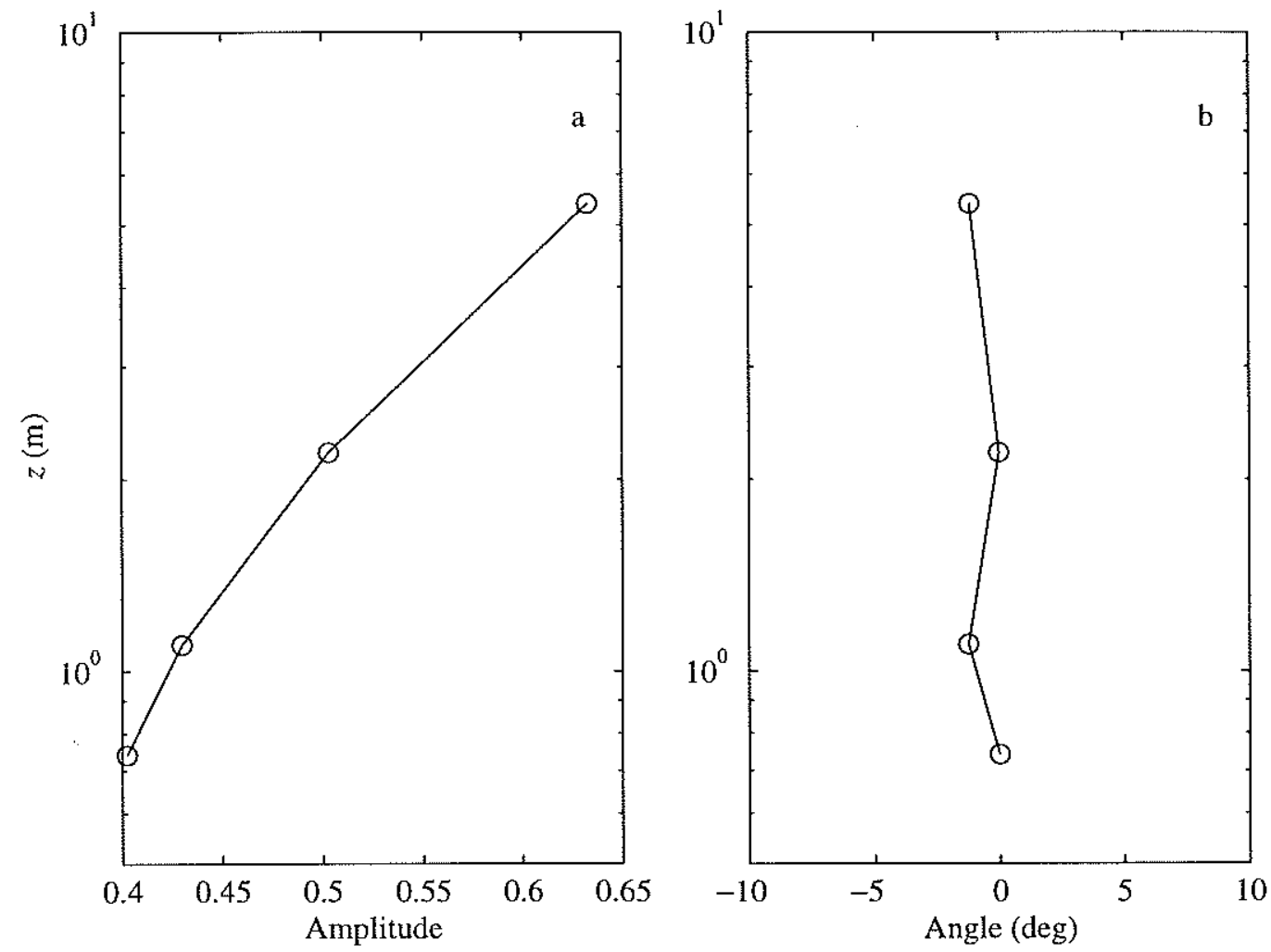

Figure 5.2: The amplitude (a) and angle (b) of the lowest temporal EOM of mean horizontal velocity as a function of height above bottom $z$. 
particular, the response to Hurricane Edouard caused substantial mixing throughout the water column. Early in the deployment, the baroclinic component of the semi-diurnal tide was significant. After the mixing caused by Hurricane Edouard inertial oscillations and the diurnal tide contributions were relatively larger, producing a complicated velocity record (Figure 5.3c). For the most part, the bottom boundary layer is well mixed, as seen in the sound speed records from the top and bottom sensors of the BASS array (Figure 5.3c). There are a few of periods of near-bottom stratification centered on days 255,259 and 268 during which strong stratification was apparently advected into the area.

\subsubsection{Flux Estimates}

In this section, we present results describing the time variability and vertical structure of the direct measurements of the fluxes of momentum and sound speed. We also consider indirect estimates of the sediment-induced buoyancy flux.

A time series of eastward Reynolds stress at four elevations between 0.7 and $3.3 \mathrm{~m}$ above the bottom (Figure 5.4a) indicates that stress estimates at the different heights are very well correlated. The variability in the time series is largely tidal but it is modulated by the strength of the sub-tidal current (see Figure $5.3 \mathrm{~b}$ ). The largest Reynolds stresses occur during the period of strong westward mean flow following Hurricane Edouard, days 246 to 250 , in which the Reynolds stresses attain a magnitude of approximately $0.15 \mathrm{~Pa}$, corresponding to $u_{*}$ values of $0.012 \mathrm{~m} / \mathrm{s}$. The strong correlation between the stress time series at the different heights and the quantitative closure of the turbulent kinetic energy budget [Shaw et al., submitted] lends confidence to the estimates.

Time series of sound speed flux at the same four elevations indicate that the estimates 

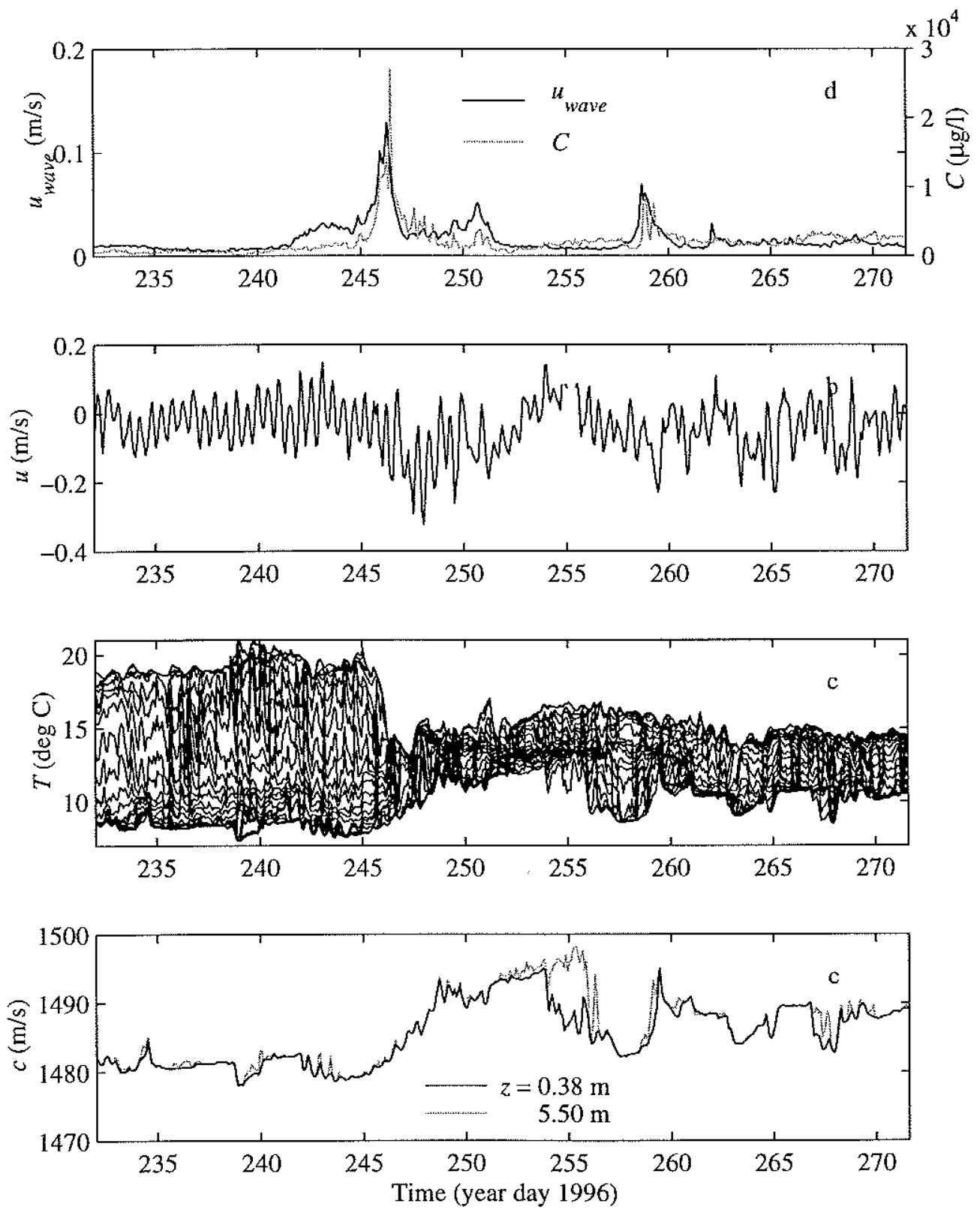

Figure 5.3: Time series of Reynolds averaged wave orbital velocity at $1.1 \mathrm{~m}$ above the bed and sediment concentration at $2 \mathrm{~m}$ above the bed (a), eastward velocity at $1.1 \mathrm{~m}$ above the bed (b), temperature throughout the water column (c) and sound speed at 0.4 and $5.5 \mathrm{~m}$ above the bottom (d) from the six-week long bottom tripod deployment. The temperature data are from throughout the water column on the central WHOI mooring. The wave orbital velocity is estimated by integrating the area below the observed wave peak in burst velocity spectra. And the sediment concentration is from a trasmissometer on a nearby bottom tripod. 
at different heights are also well correlated. The sound speed flux has the expected negative sign, corresponding to a downward sound speed flux and, under the usual temperature/salinity conditions at the site, a downward heat flux and an upward buoyancy flux. As expected, the peaks in sound speed flux are synchronous with strong Reynolds stress events, indicating that the peaks in Reynolds stress are responsible for the mixing of sound speed. Often, the sound speed flux is significant when the near-bottom sound speed gradient is large (days 255-259 and 268) suggesting that the source of the sound speed flux is the horizontal advection of stratification. During other periods (days 246-251 and 265) there are large sound speed fluxes when the bottom water is fairly well-mixed and the Reynolds stress is particularly large, suggesting that the source of the sound speed flux is mixed layer entrainment. The magnitude of the largest sound speed fluxes, approximately $2 \times 10^{-4} \mathrm{~m}^{2} \mathrm{~s}^{-2}$, roughly corresponds to a heat flux of $200 \mathrm{~W} / \mathrm{m}^{2}$, a value that is comparable to the heat flux caused by solar insolation in the upper ocean [e.g., Price et al., 1986].

As with the Reynolds-averaged horizontal velocity, we characterize the vertical structure of the flux estimates with EOM's. The lowest complex EOM of the stress time series, accounting for $86 \%$ of the observed variability, indicates that the stress is nearly constant to a height of $3.3 \mathrm{~m}$ above the bottom and then decreases with increasing the height (Figure 5.5a). There is little or no veering observed in the lowest stress EOM, consistent with the structure of the mean horizontal velocity. The EOM in Figure 5.5a is most representative of the periods of large velocity; the lowest mode computed from a subset of the data with small mean velocities (less than $0.1 \mathrm{~m} / \mathrm{s}$ at $1.1 \mathrm{~m}$ above the bottom) indicates that the stress decreases with distance from the bottom more rapidly than that shown in Figure 5.5a. This is consistent with the idea that we can estimate the height of the boundary layer based on 

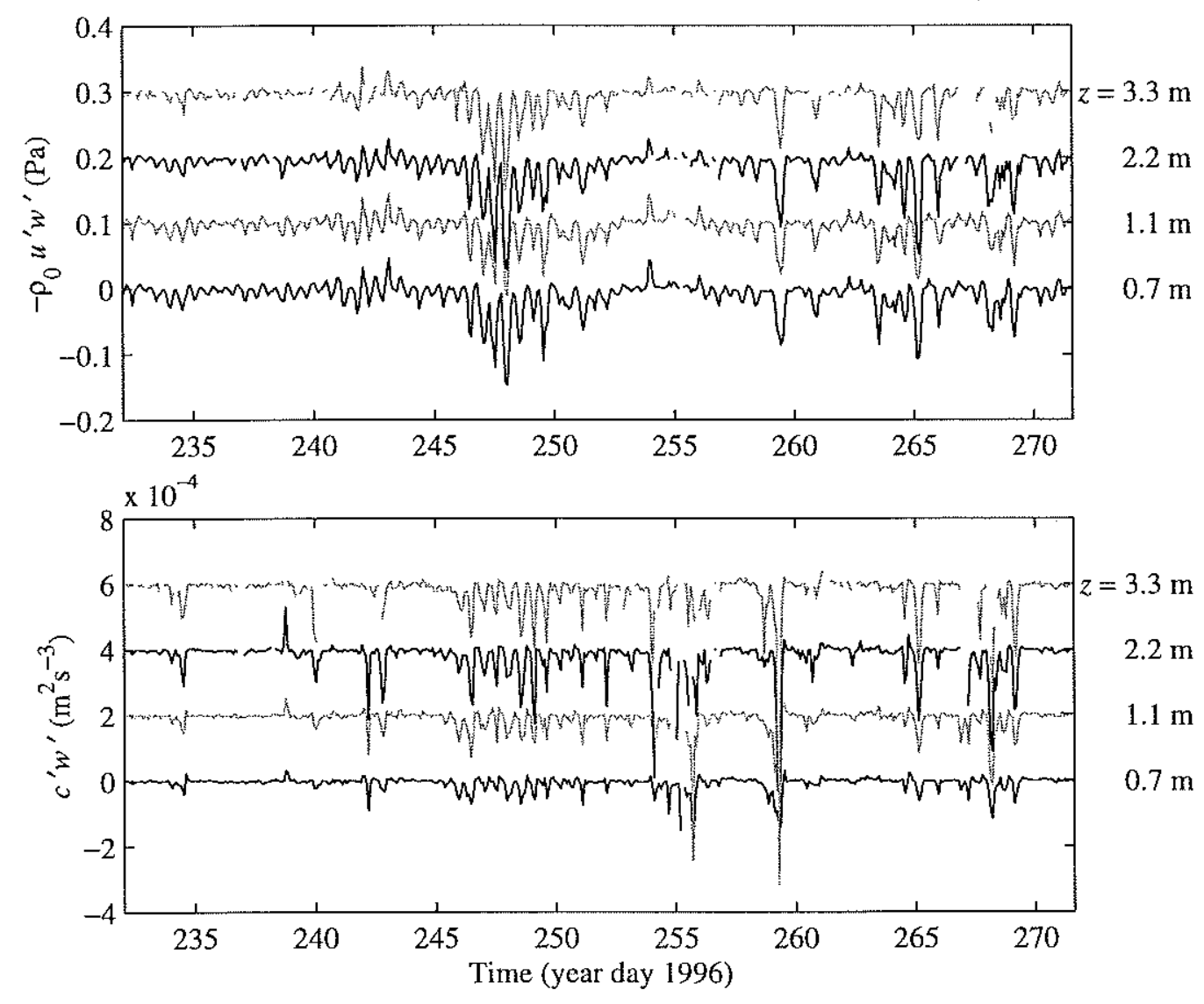

Figure 5.4: Time series of the eastward component of Reynolds stress (a), and the sound speed flux (b) at heights of $0.7,1.1,2.2$, and $3.3 \mathrm{~m}$ above the bottom. The time series from different heights are offset vertically by equal amounts. 

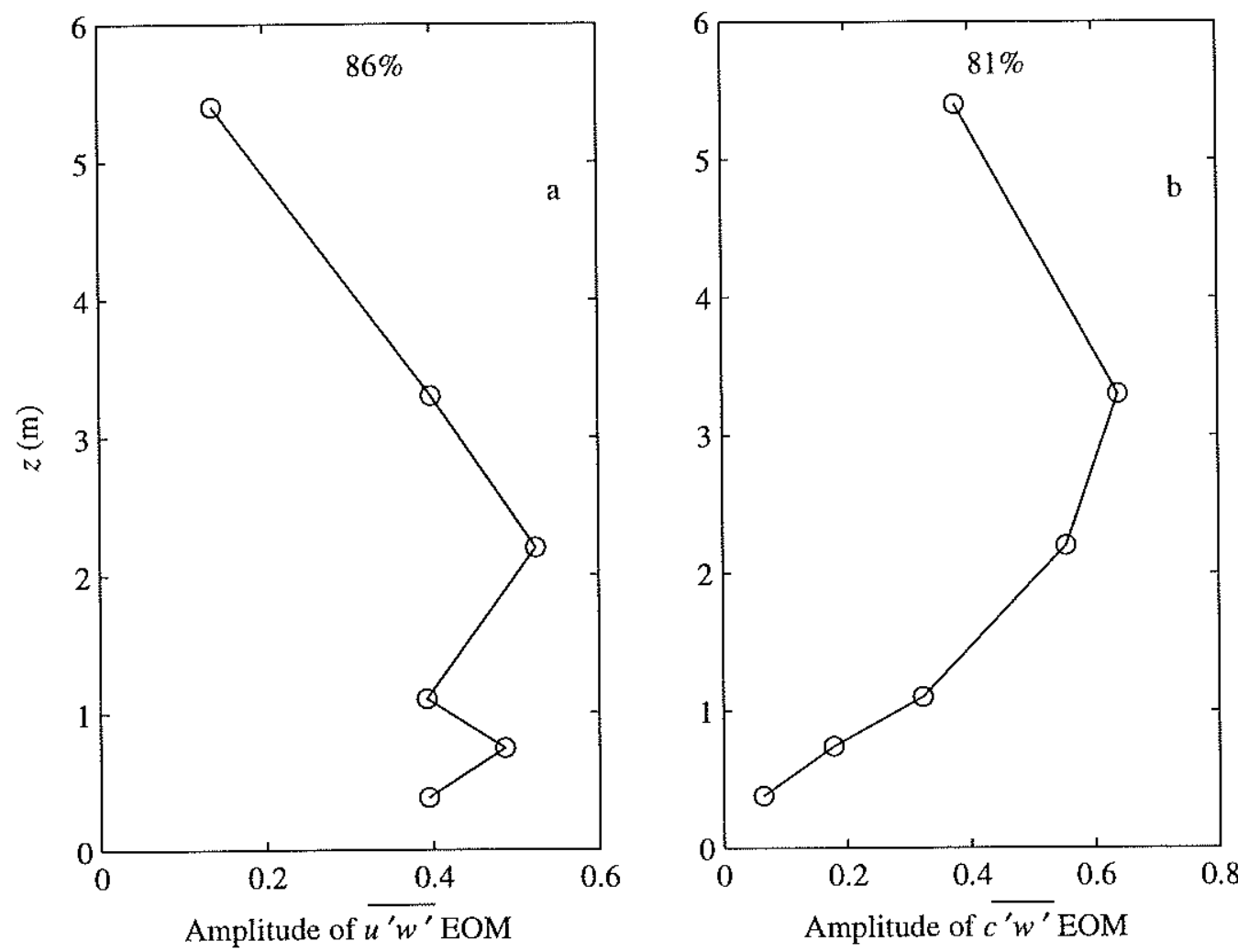

Figure 5.5: Amplitude of the lowest complex EOM for vertical momentum flux (a) and the lowest EOM for sound speed flux (b).

the intercept of the vertical stress profile.

The lowest EOM of the sound speed flux (Figure 5.5b), accounting for $81 \%$ of variance, contains more structure than the corresponding result for stress. The vertical structure is consistent with a buoyancy flux production that has a maximum in the upper portion of the boundary layer and demonstrates that the traditional surface layer MO similarity hypothesis, in which the surface value of the heat flux serves as the temperature scale, is not appropriate for the oceanic bottom boundary layer.

The sediment-induced buoyancy flux estimated from the trasmissometer data (not shown) indicates that the dynamical significance of the suspended sediment is limited to brief peri- 
ods when hurricanes passed near the site. During the hurricanes, the MO length calculated from the sediment flux $L_{\text {sed }}$ was of the same order as $z$ at the positions of the lowest sensor (indicating the buoyancy forcing due to suspended sediment was important). However, for the majority of the deployment, $z / L_{\text {sed }}<<1$. Due to uncertainties in the sediment flux estimates (which are model dependent), we do not include the effect of sediment-induced buoyancy flux in the results that are presented in the next section, noting that the effect of the sediment stratification was significant only briefly.

\subsubsection{Dimensionless Gradients}

Before presenting the dimensionless gradient results, we briefly discuss the time variability of the length scale ratios $z / L$ and $z / \delta$. A time series of the stability parameter $z / L$ at $0.9 \mathrm{~m}$ above the bed (Figure $5.6 \mathrm{a}$ ) indicates that, close to the bottom, the effect of local stratification was significant at times. For example the results of Businger et al. [1971] indicate that $z / L=0.2$ is large enough to make $\phi_{m}=2$. In particular, the periods of strong stratification identified in Figure $5.3 \mathrm{c}$ contain the largest values of the stability parameter.

Time series measurements of the height of the mixed layer and mechanical boundary layer (Figure 5.6b) indicate that the two quantities are roughly consistent in magnitude, with typical boundary layer heights of 5-10 m, but are poorly correlated. It is not surprising that the two boundary layer heights estimates are poorly correlated since they are measuring different aspects of the boundary layer. It is reassuring though that the mechanical boundary layer height estimates tend to be lower than the mixed layer height estimates and that the magnitudes of the two are generally consistent. The occasional gaps in the mechan- 

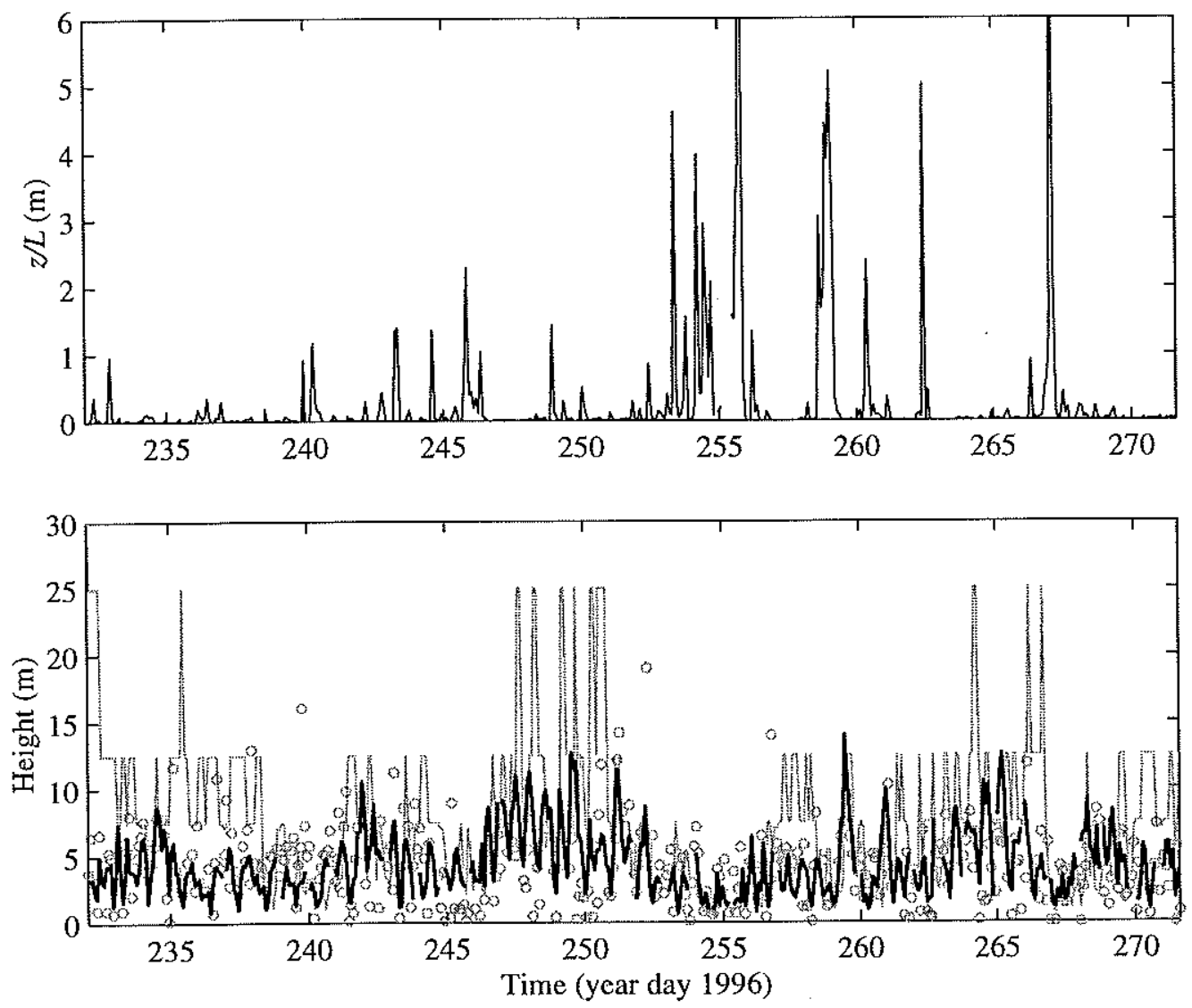

Figure 5.6: Time series of the stability parameter estimated at $0.9 \mathrm{~m}$ above the bed (a) and estimates of mixed layer and mechanical boundary layer height and a mixed layer height scale $\delta_{Z}$ (b). In (b) the mixed layer height estimates are represented by the gray line, the mechanical boundary layer estimates are represented by the gray circles and the Zilitenkivich mixed layer height scale is represented by the dark line. 
ical height time series estimate correspond to bursts with negative extrapolated $z$-intercepts caused by either measurement error or unusual vertical stress structure driven by mean flow accelerations or baroclinic pressure gradients. A time series of $\delta_{Z}$ is nearly bracketed by the two estimates of boundary layer height (Figure 5.6b). The standard planetary boundary layer height scale for neutral conditions $k u_{*} /(\omega-f)$ is 3-4 times larger than $\delta_{Z}$, indicating that the interior stratification plays an important role in setting the scale of the bottom boundary layer at the CMO site.

As a description of the temporal variability, we present time series of raw and low-passed filtered (using pl64 [Limeburner, 1985]) eddy diffusivity estimates at $1.1 \mathrm{~m}$ above the bed (Figure 5.7). The momentum diffusivity is greater than the sound speed diffusivity on average and the $k_{h}$ estimates are more highly variable. The filtered $k_{m}$ estimates vary between $10^{-4}$ and $10^{-2} \mathrm{~m}^{2} \mathrm{~s}^{-1}$ and the filtered $k_{h}$ estimates vary between $10^{-5}$ and $10^{-2} \mathrm{~m}^{2} \mathrm{~s}^{-1}$. Obtained in log space, the means and standard deviations of the $k_{m}$ and $k_{c}$ time series are $7 \times 10^{-4} \times \div 4 \mathrm{~m}^{2} \mathrm{~s}^{-1}$ and $2 \times 10^{-4} \times \div 5 \mathrm{~m}^{2} \mathrm{~s}^{-1}$, respectively. Time series of eddy diffusivity at the other heights contain similar variability.

Some of the variability in the time series of eddy diffusivities (Figure 5.7) is due to measurement error. The largest errors occur when the fluxes or gradients are small and not clearly resolved. To avoid contaminating the results of the dependency of the dimensionless gradients on $z / L$ and $z / \delta$ presented below, we limit our attention to periods when the mean velocity at $1.1 \mathrm{~m}$ above the bottom is greater than $0.1 \mathrm{~m} / \mathrm{s}$, which ensures that the momentum fluxes and gradients are measured adequately (see Discussion). Additionally, for results involving $\phi_{h}$, we discard bursts for which $\overline{c^{\prime} w^{\prime}}>-10^{-5} \mathrm{~m}^{2} \mathrm{~s}^{-2}$ in an attempt to ensure that the flux and gradient of sound speed are resolved. Furthermore, to make 

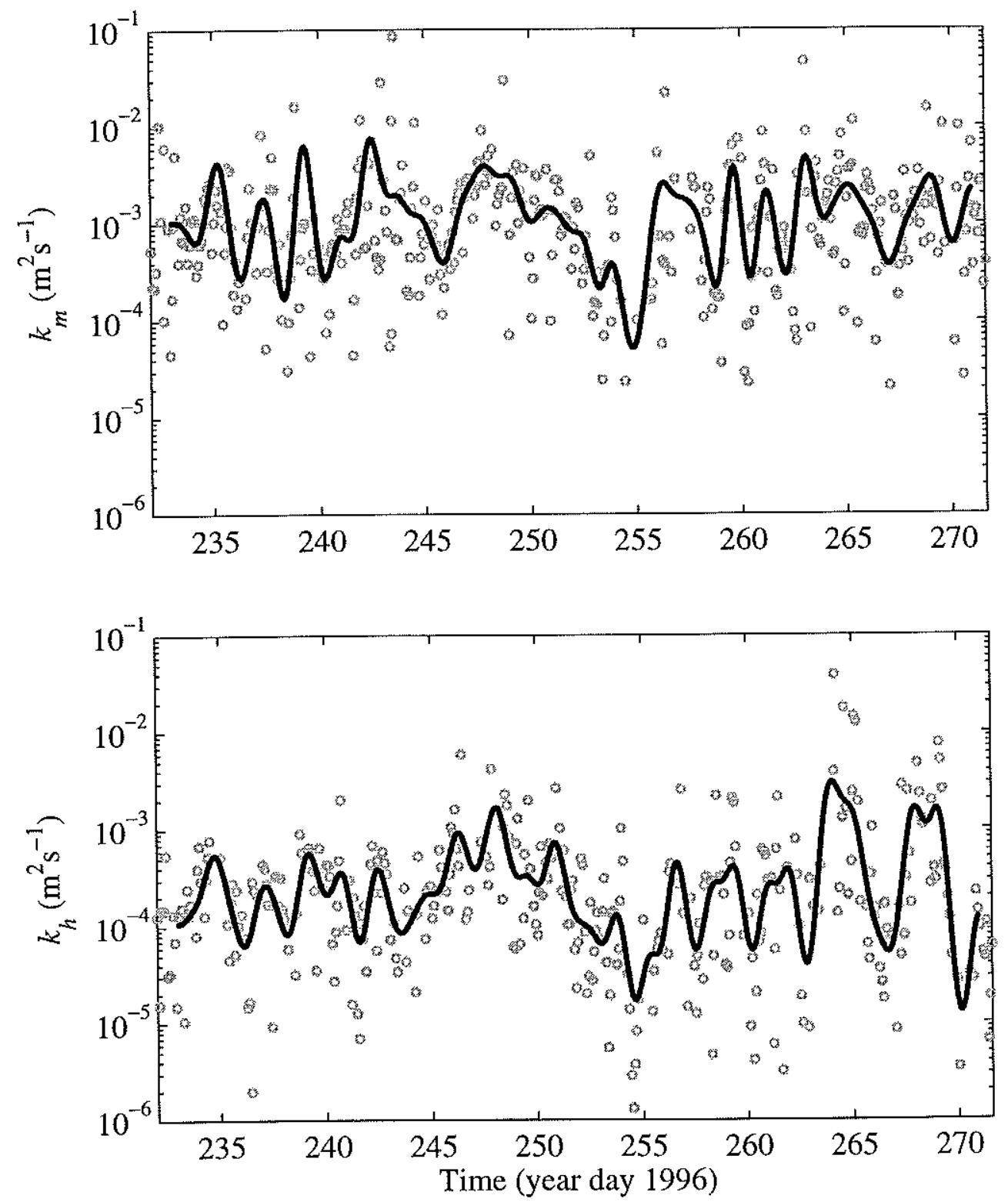

Figure 5.7: Time series of $k_{m}$ (a) and $k_{c}$ (b) at $1.1 \mathrm{~m}$ above the bed. Raw estimates are represented by open circles and low-pass filtered time series are indicated with solid lines. 
the results more clear, we present scatter plots of the dimensionless gradients averaged in bins of $z / L$ and $z / \delta$. The parameter $z / \delta$ is significant throughout the deployment (Figure $5.6 \mathrm{~b})$, making the isolation of the effects of stratification from those of boundary layer height impossible. However, the effect of stratification is significant intermittently, so the effect of boundary layer height may be partially isolated from that of stratification. When considering, the effect of $z / \delta$, we require that $z / L$ is less than $1 / 4$.

Figure $5.8 \mathrm{a}$ is a scatter plot of bin-averaged $\phi_{m}$ as a function $z / L ; 200$ estimates at each height meet the criterion introduced above and the standard error of the estimate for each bin is represented with a solid vertical line. The dependence of $\phi_{m}$ on the stability parameter at the lowest elevation, $0.9 \mathrm{~m}$, is quantitatively consistent with surface layer MO similarity results $\left(\phi_{h}=0.74+4.7 z / L\right.$ for $z / L<1$ [Businger et al., 1971]). At the upper two elevations, 1.7 and $3.8 \mathrm{~m}$, the dependence of $\phi_{m}$ on the stability parameter is qualitatively consistent with the atmospheric results, however for neutral conditions, $\phi_{m}$ approaches values of approximately 2 and 3, respectively. The bin averages for $z / L>1$ do not agree with atmospheric results, but these points also correspond to times when $z / \delta$ was large. Local MO scaling does not collapse the individual $\phi_{m}$ estimates onto a single curve.

Figure $5.8 \mathrm{~b}$ is a scatter plot of bin-averaged $\phi_{h}$ as a function of $z / L$. Depending on the height of measurement, 80 to 90 data points meet the established criteria. The $\phi_{h}$ estimates are more scattered than the $\phi_{m}$ estimates. The negative values observed at small $z / L$ must be caused by negative values of sound speed gradient. The measurements are qualitatively consistent with semi-empirical atmospheric results $\left(\phi_{m}=1+4.7 z / L\right.$ for $z / L<1$ [Businger et al., 1971]), but they are quantitatively different at all elevations and, like measurements of $\phi_{m}$, they do not collapse onto a single curve. 

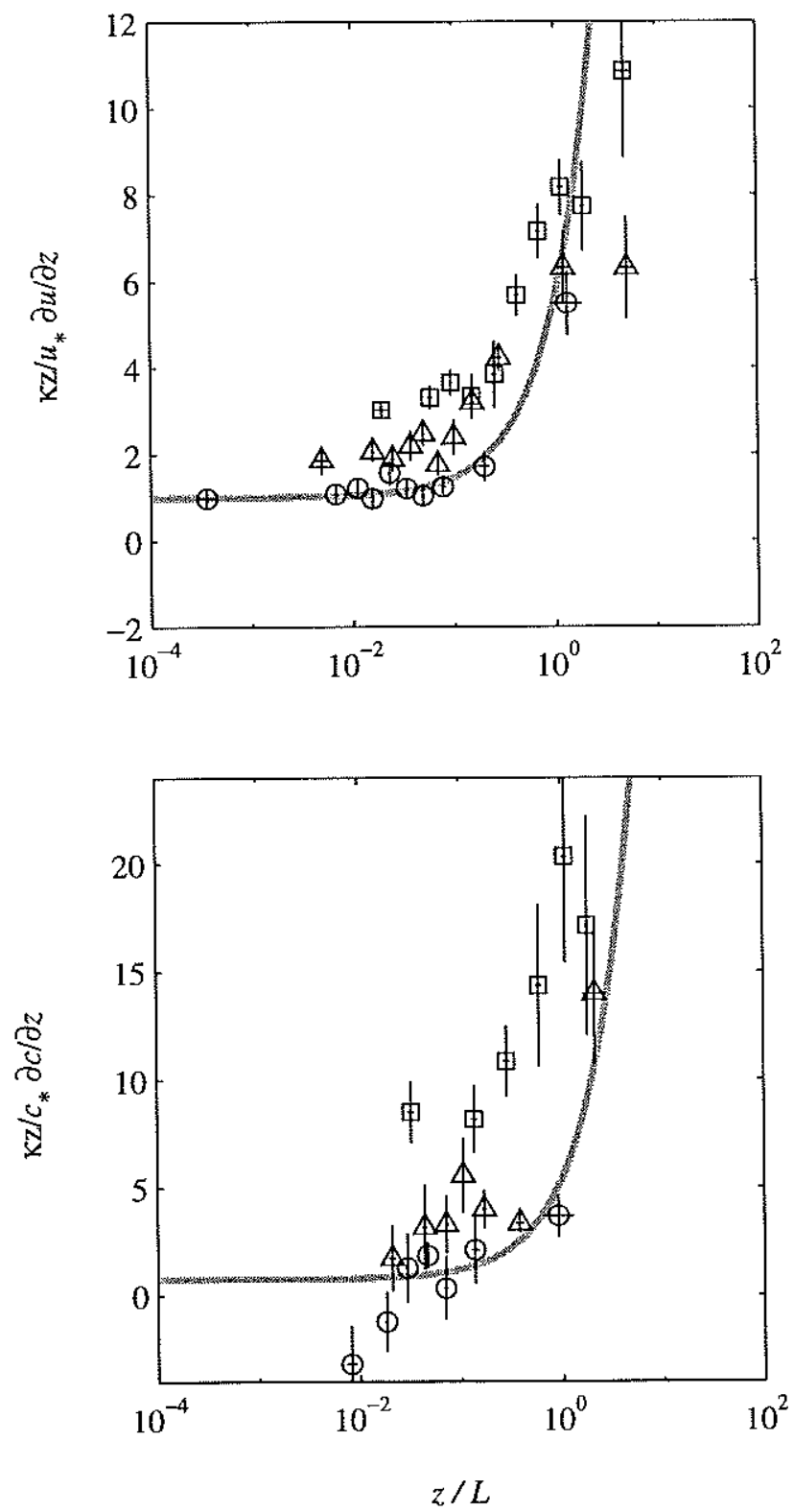

Figure 5.8: Scatter plot of bin-averaged dimensionless gradients $\phi_{m}$ (a) and $\phi_{c}$ (b) as functions of the stability parameter $z / L$ at elevations of $0.9,1.7$, and $3.8 \mathrm{~m}$ represented by circles, triangles, and squares, respectively. The solid vertical lines are the standard error for each bin. The solid gray lines are the empirical formulae of Businger et al. [1971]. 
The failure of local MO scaling to collapse the dimensionless gradient estimates suggests that boundary layer height is a potentially significant length scale. In order to be consistent with the results of Coles (see below), the dimensionless gradients presented in Figure 5.9 are calculated with an estimate of the bottom, as opposed to local, shear velocity. A bin-averaged scatter plot of the 50 to $120 \phi_{m}$ data points at each height that satisfy the established criteria indicates that variation in $z / \delta$ can account for the failure of local MO theory to collapse $\phi_{m}$ estimates (Figure 5.8a). As a function of $z / \delta$, the $\phi_{m}$ estimates are also distributed in a manner that is qualitatively similar to the law of the wake proposed by Coles [see Monin and Yaglom, 1971]

$$
\phi_{m}=1+\Pi \frac{\pi z}{\delta} \sin \left(\frac{\pi z}{\delta}\right)
$$

where $\Pi$ is an empirical constant found to be approximately 0.55 in laboratory boundary layer flows [Monin and Yaglom, 1971]. Obviously, the growing boundary flows to which Coles applied the law of the wake are not directly analogous to the bottom boundary layer in which the height is limited by stratification and rotation. The oceanic $\phi_{m}$ estimates increase more rapidly with $z / \delta$ than the laboratory results and the magnitude of the wake effect is approximately two times greater for the oceanic measurements than the laboratory measurements.

The dimensionless sound speed gradient estimates display a strong dependence on $z / L$ (Figure $5.9 \mathrm{~b}$ ). At each height, 25 to 75 bursts meet the established criteria and are included in the bin-averaging. As in Figure $5.8 \mathrm{~b}$, there are measurement problems with the sound speed gradient at low values of $z / \delta$. The $\phi_{h}$ estimates tend to increase monotonically with 

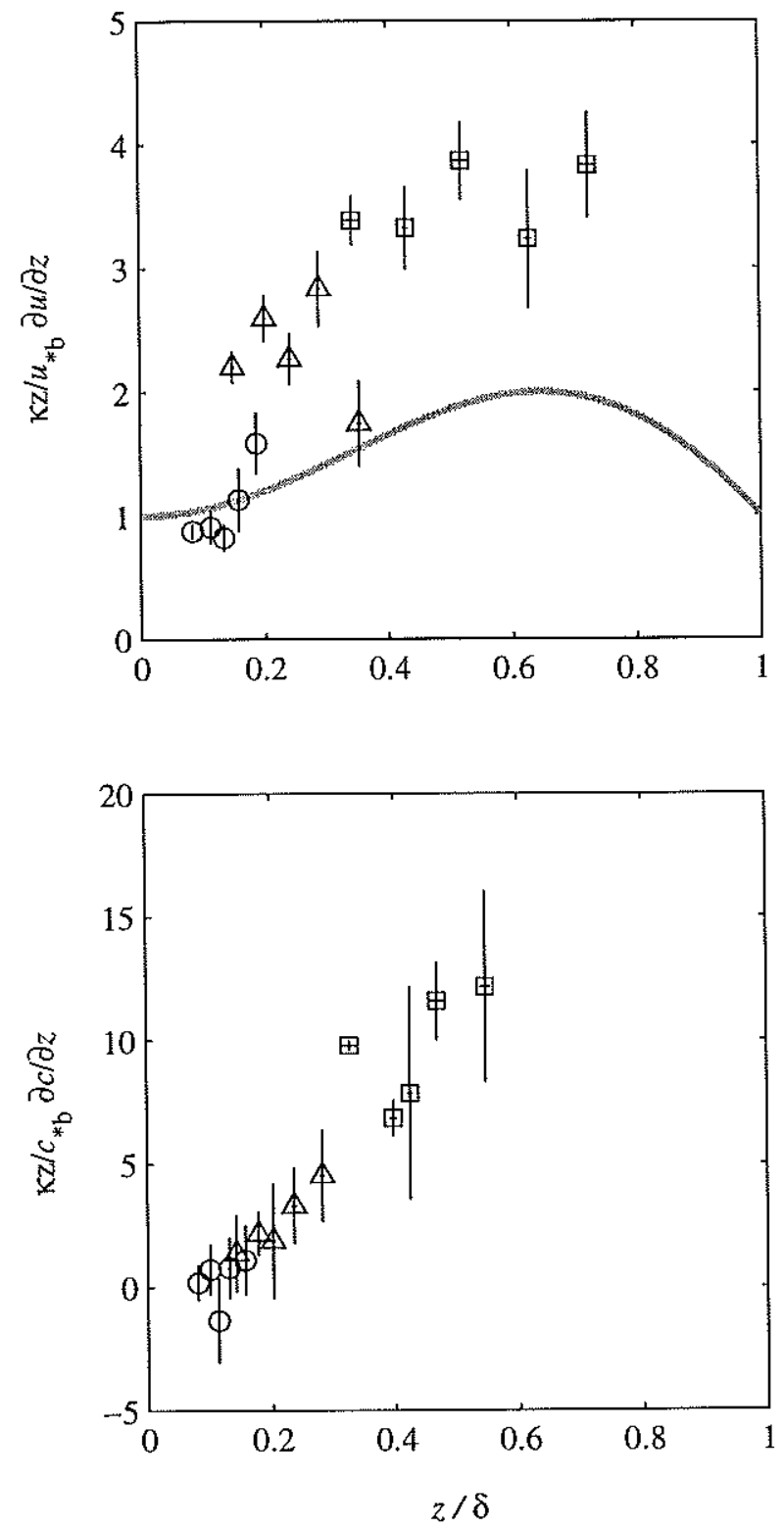

Figure 5.9: Scatter plots of bin-averaged dimensionless gradients $\phi_{m}$ (a) and $\phi_{c}$ (b) as a function of $z / \delta$. Symbols are as in Figure 5.8. The solid gray line in (a) is the empirical law of the wake of Coles [see Monin and Yaglom, 1971]. To be consistent with the scaling used in the law of the wake, the bottom shear stress is used. 
increasing $z / \delta$ and the estimates are arguably more organized in terms of $z / \delta$ than $z / L$. For small valuse of $z / \delta$ (less than approximately 0.15 ), the $\phi_{h}$ estimates tend be less than one.

\subsection{Discussion}

There is a well-defined drag law between the mean velocity at $1.1 \mathrm{~m}$ above the bed and the bottom friction velocity estimated from the stress estimates of the bottom four BASS sensors (Figure 5.10) The drag coefficient defined by $u_{*}^{2}=C_{D 110} u^{2}$ determined by regression is $1.5 \times 10^{-3}$ and is very close to the values for smooth turbulent flow [Monin and Yaglom, 1971]. The standard deviation of the errors in the fitted drag law are up to $30 \%$ of the values of the stress estimate. This represents an upper bound on the random error in the stress estimates because there is real physical variability in the drag coefficient associated with stratification, finite boundary layer thickness and other physical processes. The scatter in the stress estimates increases for values of the mean velocity less than $0.1 \mathrm{~m} / \mathrm{s}$, justifying the cutoff at that value used in the preceding section.

The results presented in Figures 5.8a and 5.9a allow us to map out roughly the different scaling regimes that occur for $\phi_{m}$ in the stably stratified shelf bottom boundary layer in the parameter space of $z / L$ and $z / \delta$ (Figure 5.11). For $z / \delta$ less than approximately 0.2 , the results indicate that local MO theory can be expected to hold and the important length scales are $z$ and $L$. There are two limiting cases within the MO regime. For $z / L$ less than approximately 0.1 , the effects of stratification are not important and the Prandtl-Karman law of the wall is satisfied. The law of the wall is only satisfied at the lowest measurement 


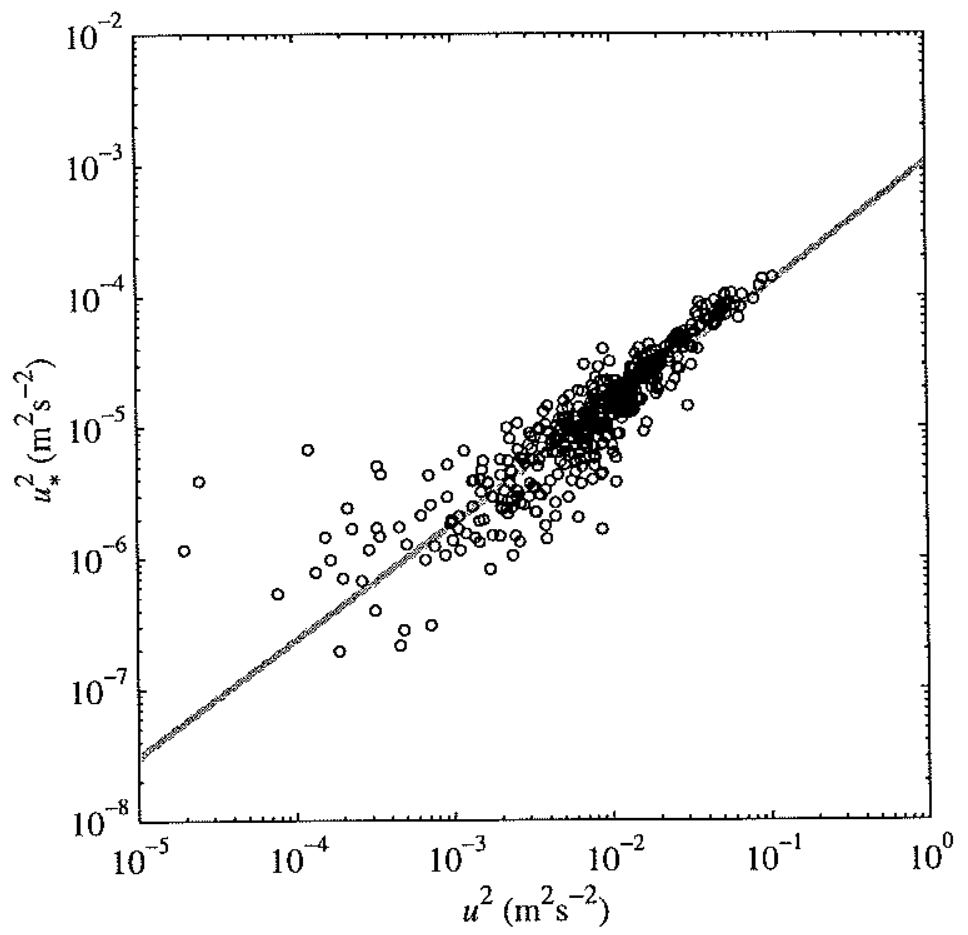

Figure 5.10: Drag relationship for mean velocity at $1.1 \mathrm{~m}$ above the bed. The solid line is the drag relationship for the logarithmic region of smooth turbulent flow [Monin and Yaglom, 1971]. 


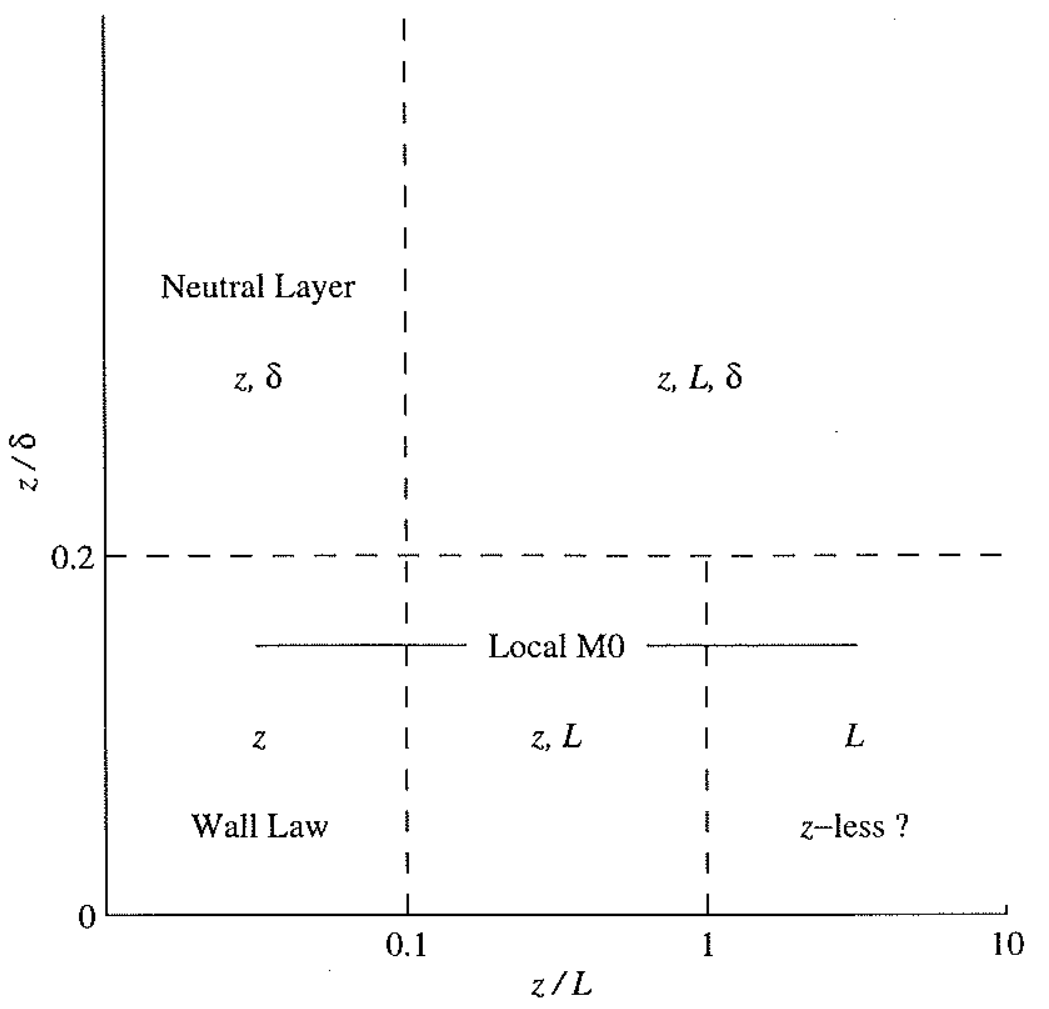

Figure 5.11: Schematic diagram of the turbulence scaling regimes likely to be found in the bottom boundary layer.

height $(0.9 \mathrm{~m})$ during the periods of strongest mechanical forcing. At the other extreme, $z / L$ greater than approximately one, it is expected that $z$-less scaling [Wyngaard, 1973] should be valid. This regime was not resolved with our measurements. In general, all three length scales are important when $z / \delta$ and $z / L$ are appreciable. For the case of $z / L$ less than about 0.1 , the results (Figure 5.9a) suggest that a regime, termed the neutral layer, may exist where only $z$ and $\delta$ are important.

We can interpret the dimensionless shear results in terms of the turbulent mixing length for momentum. The results suggest that the mixing length is strongly influenced by $z / \delta$ in addition to stratification. We find that the mixing length is less than $z$ when $z / \delta$ is not 
small. This result is inconsistent with similar measurements obtained in the stable atmospheric boundary layer by Nieuwstadt [1984], who found that local MO scaling collapsed dimensionless gradients at heights throughout the boundary layer. Since Nieuwstadt's experiment, large-eddy and direct numerical simulations of the stable atmospheric boundary, have also indicated local MO scaling is successful [Wyngaard, 1992]. The height of the stable atmospheric boundary layer is not usually limited by overlying stratification [Wyngaard, 1992] and this may be the reason for the discrepancy between the present results and the previous atmospheric work. In the present study, the overlying stratification strongly affected the dynamics of the bottom boundary layer: the mixed layer scale is $3-4$ times smaller than the planetary boundary layer height scale and there is little or no veering in the mean currents. The idea of overlying stratification providing a strong constraint on the length scale of eddies is a physically appealing concept. If this is the case, the mixed layer height, rather than the mechanical height of the boundary layer may be the most relevant parameter.

It is illustrative to discuss the results presented in Figures 5.8 and 5.9 in terms of turbulent Prandtl number. Estimating the turbulent Prandtl number is difficult because it is a function of four variables with random variability and results are sensitive to the manner in which they are averaged. We believe that the most meaningful estimates are obtained from the bin-averaged local $\phi$ estimates and the use of (5.13). Plotting $\operatorname{Pr}_{t}$ estimates obtained in this manner as a function of $z / L$ and $z / \delta$ (Figure 5.12) indicates that, although the error bars are large and there is considerabe scatter, the variability in $P r_{t}$ is more closely related to the height of the boundary layer than local stratification. Values of $\operatorname{Pr}_{t}$ tend to be less than one for $z / \delta$ less than approximately 0.1 . For large values of $z / \delta, P r_{t}$ 
hangs around a value of two. Interpreted in terms of mixing lengths, the results suggest that $l_{h}>l_{m}$ for small $z / \delta$ but that $l_{h}<l_{m}$ for $z / \delta \approx 1$. A more appealing physical explanation for the small $P r_{t}$ values for $z / \delta<0.1$ is non-local transport, an effect not considered in the similarity theory and which invalidates the mixing length analogy. The transport mechanism we hypothesize is turbulent diffusion of sound speed flux, represented by the term $\partial \overline{c^{\prime} w^{\prime 2}} / \partial z$ in the equation for $\overline{c^{\prime} w^{\prime}}$ [e.g., Lumley and Panofsky, 1964]. Non-local transport is also consistent with previous results in which the sound speed variance budget was not balanced locally [Shaw et al., submitted]. This mechanism may account for small $P r_{t}$ values near the bottom, it remains unclear, however, why $P r_{t}$ is larger than one in the outer part of the boundary layer. The present results are also inconsistent with previous atmospheric results [Businger et al., 1971].

The results presented in the previous section indicate that large errors are likely if the law of the wall is always assumed to hold in the continental shelf boundary layer. As a corollary, use of the inertial subrange technique will also be subject to error. The results also indicate that care must be taken interpreting observed deviations from logarithmic profiles in terms of stratification or acceleration effects. Regarding turbulence closure models, the results indicate that the turbulent length scale should be formulated to include a dependence on $z / \delta$. If our hypothesis about non-local transport of sound speed can be verified, it implies that the simplifications made, for example, in the Mellor-Yamada Level III closure are not valid, i.e. models should include the turbulent diffusion of scalar variance and scalar flux. 

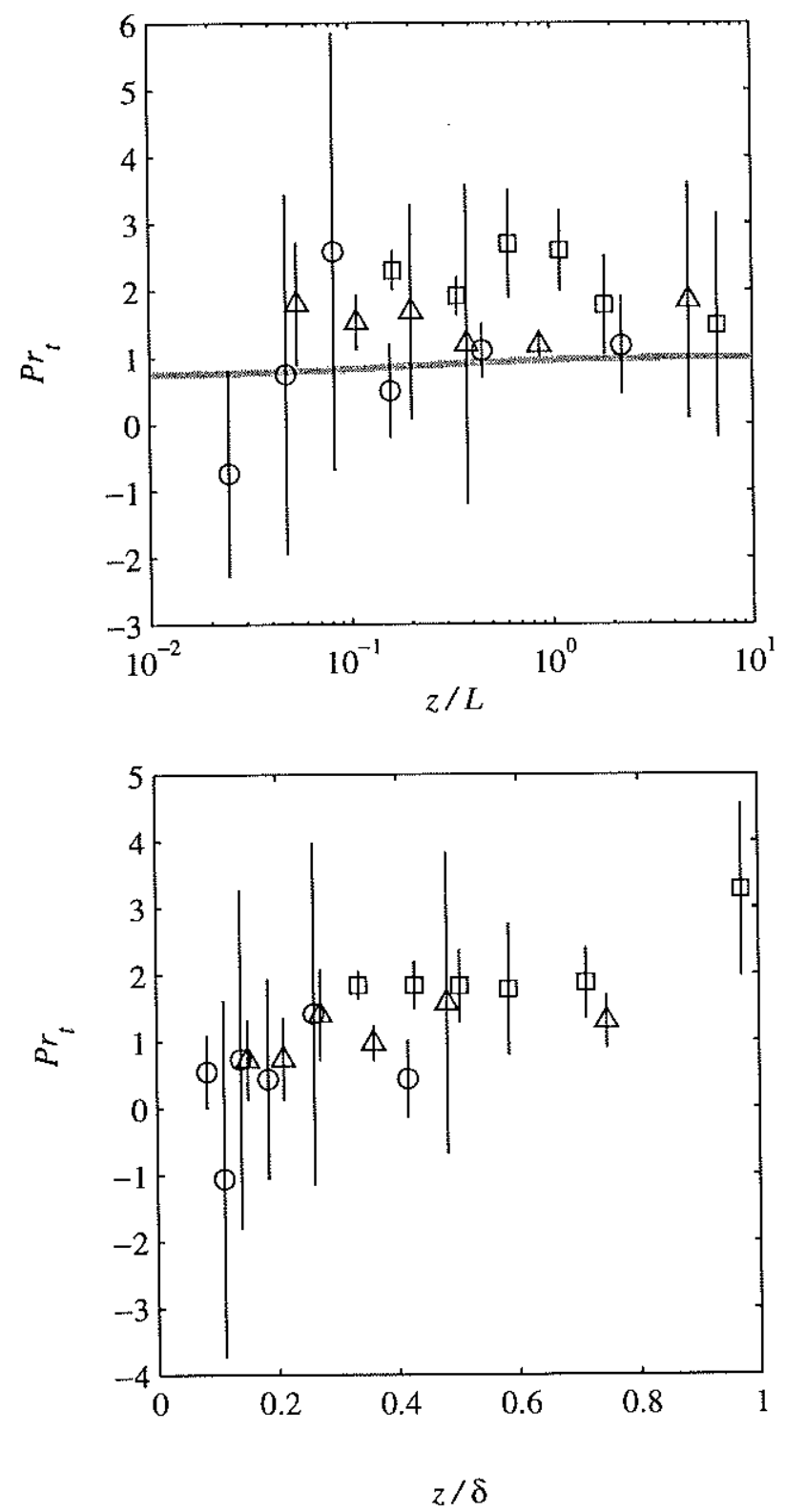

Figure 5.12: Scatter plot of bin averaged turbulent Prandtl numbers $P r_{t}$ as a function of $z / L(\mathrm{a})$ and $z / \delta(\mathrm{b})$ at elevations of $0.9,1.7$, and $3.8 \mathrm{~m}$ represented by circles, triangles, and squares, respectively. The solid gray line in (a) is the empirical formula of Businger et al. [1971]. 


\subsection{Summary and Conclusions}

In this paper we have presented dimensionless profile of velocity and sound speed obtained from heights of approximately 1 to $4 \mathrm{~m}$ above the bottom in the bottom boundary layer of the New England Shelf from direct measurement of the required quantities. The results were presented as function of the Monin-Obukhov stability parameter and the ratio of the height above bottom to height of the boundary layer.

The results indicate the following conclusions. The forcing at the experimental site was predominantly tidal, with the largest stresses occurring during periods of westward sub-tidal current. The source of the buoyancy flux was from mixed layer entrainment, horizontal advection of stratification and, for brief periods during large storms, sediment resuspension. At the lowest measurement elevation, $0.9 \mathrm{~m}$ above the bottom, local MoninObukhov similarity theory was found to be valid. At higher measurement positions, local Monin-Obukhov scaling failed to explain the observed variability. The variability can be explained in terms of the ratio of the height above bottom to height of the boundary layer. We interpret the results to mean that the height of the mixed layer imposes of strong constraint on the turbulent mixing length. Estimates of the turbulent Prandtl number are weakly correlated with the ratio of the height above bottom to height of the boundary layer, we interpret part of the variability as an effect of non-local transport of sound speed. The results indicate large errors are possible in the application of the logarithmic profile technique in the continental shelf bottom boundary layer. The results also suggest that the importance of turbulent diffusion terms in turbulence closure should be reevaluated. 


\section{Bibliography}

J. A. Businger, J. C. Wyngaard, Y. Izumi, and E. F. Bradley. Flux-profile relationships in the atmospheric surface layer. J. Atmos. Sci, 28:181-189, 1971.

H. Charnock. Tidal friction from currents near the seabed. Geophys. J. R. Astron. Soc., 2: $215-221,1959$.

D. Coles. The law of the wake in the turbulent boundary layer. J. Fluid Mech., 2:191-226, 1956.

R. E. Davis. Predictability of sea surface temperature and sea level pressure anomalies over the north pacific ocean. J. Phys. Oceanogr., 6:249-266, 1976.

T. D. Dickey, G. C. Chang, Y. C. Agrawal, A. J. III Williams, and P. S. Hill. Sediment resuspension in the wakes of hurricanes edouard and hortense. ???, submitted.

J. J. Fredericks, J. H. Trowbridge, A. J. Williams, G. Voulgaris, and W. J. Shaw. Fluid mechanical measurements within the bottom boundary layer during coastal mixing and optics. Technical report, Woods Hole Oceanographic Institution, 1999.

N. Galbraith, W. Ostrom, B. Way, S. Lentz, S. Anderson, M. Baumgartner, A. Pluedde- 
mann, and J. Edson. Coastal mixing and optics experiment: Mooring deployment cruise report. Technical report, WHOI, 1997.

T. F. Gross and A. R. M. Nowell. Mean flow and turbulence scaling in a tidal boundary layer. Cont. Shelf Res., 2:109-126, 1983.

A. D. Heathershaw. The turbulent structure of the bottom boundary layer in a tidal current. Geophys. J. R. Astr. Soc., 58:395-430, 1979.

K. Kawanisi and S. Yokosi. Mean and turbulence characteristics in a tidal river. Est. Coast. Shelf Sci., 38:447-469, 1994.

S. J. Lentz and J. H. Trowbridge. The bottom boundary layer over the northern california shelf. J. Phys. Oceanogr., 21:1186-1201, 1991.

R. Limeburner. Code-2: Moored array and large-scale data report. Technical report, Woods Hole Oceanographic Institution, 1985.

R. G. Lueck and Y. Lu. The logarirhmic layer in a tidal channel. Cont. Shelf Res., 17: 1785-1801, 1997.

J. L. Lumley and H. A. Panofsky. The Structure of Atmospheric Turbulence. Interscience, New York, NY., 1964.

A. S. Monin and A. M. Yaglom. Statistical Fluid Mecahnics. MIT Press, Cambridge, Mass., 1971.

F. T. M. Nieuwstadt. The turbulent structure of the stable, nocturnal boundary layer. $J$. Atmos. Sci., 41:2202-2216, 1984. 
J. F. Price, R. A. Weller, and R. Pinkel. Diurnal cycling: Observations and models of the upper ocean response to diurnal heating, cooling, and wind mixing. J. Geophys. Res., 91: $8411-8427,1986$.

T. B. Sanford and R.-C. Lien. Turbulent properties in a homogeneous tidal bottom boundary layer. J. Geophys. Res., 104:1245-1257, 1999.

W. J. Shaw and J. H. Trowbridge. The measurement of near-bottom turbulent fluxes in the presence of energetic wave motions. J. Atm. and Ocean. Tech., submitted.

W. J. Shaw, J. H. Trowbridge, and A. J. III Williams. The budgets of turbulent kinetic energy and scalar variance in the continental shelf bottom boundary layer. J. Phys. Oceangr., submitted.

J. D. Smith and S. R. McLean. Spatially averaged flow over a wavy surface. J. Geophys. Res., 82:1735-1746, 1977.

Z. Sorbjan. On similarity in the atmospheric boundary layer. Bound.-Layer Meteor., 34: 377-397, 1986.

R. L. Soulsby. The bottom boundary layer of shelf seas. In B. Johns, editor, Physical Oceanography of Coastal and Shelf Seas, pages 189-266. Elsevier, 1983.

R. L. Soulsby and K. R. Dyer. The form of the near-bed velocity profile in a tidally accelerating flow. J. Geophys. Res., 86:8067-8074, 1979.

M. T. Stacey, S. G. Monismith, and J. R. Burau. Observations of turbulence in a partially stratified estuary. J. Phys. Oceanog., submitted. 
R. W. Sternberg. Friction factors in tidal channels with differing bed roughness. Mar. Geol., $6: 243-260,1968$.

R. O. R. Y. Thompson. Stratified ekman boundary layer models. Geophys. Fluid Dyn., 5: 201-210, 1973.

J. H. Trowbridge, W. R. Geyer, M. M. Bowen, and A. J. Williams. Near-bottom turbulence mesurements in a partially mixed estuary: Turbulent energy balance, velocity structure, and along-channel momentum balance. J. Phys. Oceanogr., in press.

A. J. Williams, J. S. Tochko, R. L. Koehler, W. D. Grant, Gross T. F., and C. V. R Dunn. Measurement of turbulence in the oceanic bottom boundary layer with an acoustic current meter array. J. Atm. and Ocean. Tech., 4:312-327, 1987.

J. C. Wyngaard. On surface layer turbulence. In D. A. Haugen, editor, Workshop on Micrometeorology, pages 101-149. Amer. Meteor. Soc., 1973.

J. C. Wyngaard. Atmospheric turbulence. Ann. Rev. Fluid Mech., 24:205-233, 1992.

S. S. Zilitinkevich. On the determination of the height of the ekman boundary layer. Bound.Layer Meteor., 3:141-145, 1972. 


\section{Chapter 6}

\section{Summary}

The goal of this thesis was to obtain, reduce, and interpret the measurements required to study, as directly as possible, the mechanisms of turbulent mixing in the continental shelf bottom boundary layer. Part of the research was technical in nature, including instrument modifications and the development of a new analysis technique, and was required to achieve the scientific goals. The scientific sections of the thesis consisted of testing balances of turbulent kinetic energy and of the variance of a scalar (speed of sound) and testing turbulence closure hypotheses in the form of dimensionless gradients of velocity and speed of sound.

The work undertaken in the thesis has resulted in the following conclusions:

1) Acoustic current meters can be used to measure the turbulent fluctuations of sound speed with the required resolution to estimate scalar fluxes and dissipation rates in the coastal bottom boundary.

2) A new linear filtration and differencing technique is successful at removing surface wave contamination from estimates of turbulent fluxes of momentum and scalars.

3) The turbulent kinetic energy budget in a continental shelf field site is balanced locally, 
primarily by production and dissipation. The direct effect of stratification, quantified in terms of the flux Richardson number, is typically limited to the outer part of the boundary layer. The sound speed variance budget does not close locally and the observed imbalance is consistent with downward turbulent diffusion of scalar variance.

4) Close to the bottom the dimensionless shear is quantitatively consistent with the semi-empirical results of Monin-Obukhov similarity theory. Further from the boundary local Monin-Obukhov scaling fails and the length scale imposed by the height of the mixed layer is significant. The turbulent Prandtl number is difficult to estimate with the measurements, but the average value is greater than one.

The work performed for this thesis has resulted in the most comprehensive study of turbulent mixing in the coastal bottom boundary layer to date. The research has identified aspects of the coastal BBL that are similar to other boundary layers (especially the stable atmospheric boundary layer) and identified aspects that are unique. In particular, mixed layer entrainment is an important process that may affect the turbulent length scale and the dynamics of scalar fluctuations. More specifically, the results indicate that commonly used assumptions of microstructure studies are not valid in the coastal bottom boundary layer and that large errors may result from the indiscriminate use of the log-profile technique to estimate bottom stress. In terms of turbulent closure, the work indicates that the length scale imposed by the height of the mixed layer is important and that the dynamics of scalar fluctuations are non-local.

The research conducted for this thesis suggests several areas for further work. The direct turbulent flux estimates obtained in this thesis could be used in conjunction with measurements from the WHOI mooring array that was deployed in the same area to test 
momentum and heat budgets for the shelf BBL. The budgets could be used to put the turbulence measurements in perspective and to test if the locally measured fluxes are representative of the drag and mixing experienced by the boundary layer on a larger scale. The results indicated by the turbulence estimates obtained from the field measurements in this thesis are not definitive. Computer simulations of coastal BBL turbulence could be used to augment the present estimates. In particular, they may be useful for verifying the results and, potentially, for identifying mechanisms that lead to turbulent Prandtl numbers greater than one. The effects of internal waves on coastal BBL turbulence were not emphasized in the thesis. Energetic internal waves were present intermittently, however, and the present measurements may be useful for studying mixing in internal wave boundary layers. 